\title{
STRUCTURE-ACTIVITY RELATIONSHIPS IN NI-FE (OXY)HYDROXIDE OXYGEN EVOLUTION ELECTROCATALYSTS
}

by

ADAM S. BATCHELLOR

\section{A DISSERTATION}

Presented to the Department of Chemistry and Biochemistry and the Graduate School of the University of Oregon

in partial fulfillment of the requirements

for the degree of

Doctor of Philosophy

December 2016 


\section{DISSERTATION APPROVAL PAGE}

Student: Adam S. Batchellor

Title: Structure-Activity Relationships in Ni-Fe (Oxy)hydroxide Oxygen Evolution Electrocatalysts

This dissertation has been accepted and approved in partial fulfillment of the requirements for the Doctor of Philosophy degree in the Department of Chemistry and Biochemistry by:
Catherine Page
Chairperson
Shannon Boettcher
Advisor
Mark Lonergan
Core Member
Paul Wallace
Institutional Representative

and

Scott L. Pratt

Dean of the Graduate School

Original approval signatures are on file with the University of Oregon Graduate School.

Degree awarded December 2016 
(C) 2016 Adam S. Batchellor 


\section{DISSERTATION ABSTRACT}

Adam S. Batchellor

Doctor of Philosophy

Department of Chemistry and Biochemistry

September 2016

Title: Structure-Activity Relationships in Ni-Fe (Oxy)hydroxide Oxygen Evolution Electrocatalysts

The oxygen evolution reaction (OER) is kinetically slow and hence a significant efficiency loss in electricity-driven water electrolysis. Understanding the relationships between architecture, composition, and activity in high-performing catalyst systems are critical for the development of better catalysts.

This dissertation discusses areas both fundamental and applied that seek to better understand how to accurately measure catalyst activity as well as ways to design higher performing catalysts. Chapter I introduces the work that has been done in the field to date. Chapter II compares various methods of determining the electrochemically active surface area of a film. It further discusses how pulsed and continuous electrodepostition techniques effect film morphology and behavior, and shows that using a simple electrodeposition can create high loading films with architectures that outperform those deposited onto inert substrates. The reversibility of the films, a measure of the films transport efficiency, is introduced and shown to correlate strongly with performance. Chapter III uses high energy x-ray scattering to probe the nanocrystalline domains of the largely amorphous NiFe oxyhydroxide catalysts, and shows that significant similarities in the local structure are not responsible for the change in performance for the films 
synthesized under different conditions. Bond lengths for oxidized and reduced catalysts are determined, and show no significant phase segregation occurs. Chapter IV seeks to optimize the deposition conditions introduced in Chapter II and to provide a physical representation of how tuning each of the parameters affects film morphology. The deposition current density is shown to be the most important factor affecting film performance at a given loading. Chapter V highlights the different design considerations for films being used in a photoelectrochemical cell, and how in situ techniques can provide information that may otherwise be unobtainable. Chapter VI serves as a summary and provides future directions.

This dissertation contains previously published coauthored material. 
CURRICULUM VITAE

NAME OF AUTHOR: Adam S. Batchellor

GRADUATE AND UNDERGRADUATE SCHOOLS ATTENDED:

University of Oregon, Eugene

State University of New York at Buffalo

Clarkson University, Potsdam, New York

\section{DEGREES AWARDED:}

Doctor of Philosophy, Chemistry, 2016, University of Oregon Master of Science, Chemical Engineering, 2011, University at Buffalo

Bachelor of Science, Chemical Engineering, 2003, Clarkson University

AREAS OF SPECIAL INTEREST:

Materials Science

Science Education

Weapons Chemistry

PROFESSIONAL EXPERIENCE:

Bioenvironmental Engineer, United States Air Force, 2004-2009

Assistant Professor of Chemistry, United States Air Force, 2011-2013

GRANTS, AWARDS, AND HONORS:

Scientific Teaching Fellow, Mobile Summer Institute, University of Oregon, 2016 


\section{PUBLICATIONS:}

Batchellor, A.; Trotochaud, L.; Boettcher, S. W. In Situ Characterization of the Optical Properties of Electrocatalysts, In Advanced and In Situ Analytical Methods for Solar Fuel Materials 2015, Volume 371 of the series Topics in Current Chemistry, pp 253-324.

Batchellor, A. S.; Boettcher, S. W. Pulse-Electrodeposited Ni-Fe (Oxy)hydroxide Oxygen Evolution Electrocatalysts with High Geometric and Intrinsic Activities at Large Mass Loadings. ACS Catal. 2015, 5 (11), 6680-6689.

Burke, M. S.; Enman, L. J.; Batchellor, A. S.; Zou, S.; Boettcher, S. W. Oxygen Evolution Reaction Electrocatalysis on Transition Metal Oxides and (Oxy)hydroxides: Activity Trends and Design Principles. Chem. Mater. 2015, 27 (22), 7549-7558.

Enman, L. J.; Burke, M. S.; Batchellor, A. S.; Boettcher, S. W. Effects of Intentionally Incorporated Metal Cations on the Oxygen Evolution Electrocatalytic Activity of Nickel (Oxy)hydroxide in Alkaline Media. ACS Catal. 2016, 2416-2423.

Stevens, M. B.; Enman, L. J.; Batchellor, A. S.; Cosby, M. R.; Vise, A. E.; Trang, C. D.; Boettcher, S.W. Measurement Techniques for the Study of Heterogeneous Water Oxidation Electrocatalysts. Chem. Mater. 2016, Accepted.

Batchellor, A. S.; Kwon, G.; Tiede, D. M.; Boettcher, S. W. Domain Structures of $\mathrm{Ni}$ and $\mathrm{NiFe}(\mathrm{Oxy}) \mathrm{Hydroxide}$ Oxygen-Evolution Catalysts from X-ray Pair Distribution Function Analysis. J. Phys. Chem. C. 2016, Submitted. 


\section{ACKNOWLEDGMENTS}

I must begin by thanking the Department of Chemistry at the United States Air Force Academy for the opportunity to pursue my PhD. Your belief in my ability as both an instructor and student of chemistry has instilled in me a great deal of confidence and resolve. I look forward to returning in the future to share all that I have acquired in the past three years.

Next, I would like to thank the members of the Boettcher lab. It is the exception, not the rule, that such a large number of diverse young professionals can work tirelessly and harmoniously together. Keep the coffee going, Matt. In particular, Team OER, I am impressed at how we have gone from mentees to mentors in such a short time. I leave the lab, and the solar materials and electrochemistry field, with many unanswered questions. But I depart in good spirits, having little doubt in your ability to answer them in my absence.

I would also like to thank Dr. Shannon Boettcher. Your passion for science is infectious. Your skills as a researcher and communicator of science are impeccable. I am happy to have learned as much from a fellow '03 grad as I did, and I hope you can say the same thing.

Lastly, I would like to thank my family for all the words of encouragement. Knowing how proud everyone is of my efforts towards becoming a Doctor has been instrumental in keeping me focused and progressing. To my amazing wife Nikki, I can't properly express my gratitude for all that you have given, and will continue to give, to allow me the chance to realize my professional goals. It is through your fortitude as a wife and mother that I can be both an Airman and a Scholar. To my wonderful son, 
Beau, you are the only experiment started here in Oregon that I am truly and wholeheartedly vested in, and you are by far my greatest accomplishment to date. 
To my late friend, Marin Miljak Cizmic.

I am grateful for the time we knew each other.

Your loss is felt fully on both sides of the pond. 


\section{TABLE OF CONTENTS}

Chapter

Page

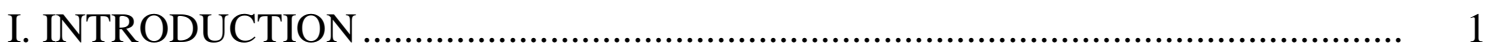

Challenges to Efficient Water Splitting ....................................................... 1

Synthesis and Structure of Ni-based OER Catalysts ...................................... 3

Characterization of Disordered Films ............................................................ 5

Summary and Bridge .......................................................................... 6

II. PULSE-ELECTRODEPOSITED NI-FE (OXY)HYDROXIDE OXYGEN

EVOLUTION ELECTROCATALYSTS WITH HIGH GEOMETRIC AND

INTRINSIC ACTIVITIES AT LARGE MASS LOADINGS ..................... 8

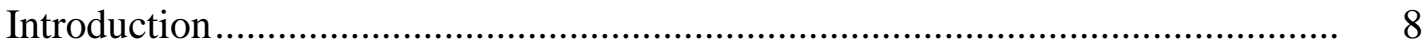

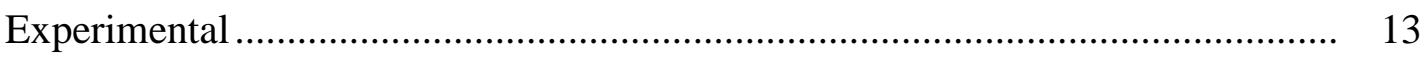

Electrode Fabrication ......................................................................... 13

Film Deposition ........................................................................ 14

Electrochemical Measurements ......................................................... 15

Film Loading and TOF Calculcations..................................................... 15

Double Layer Capacitance ................................................................. 16

Film Characterization........................................................................ 17

Results and Discussion ...................................................................... 17

Performance of Electrochemically Deposited Catalyst Films. .................... 17

Determining Loading and Effective TOF ........................................... 19

Capacitance Measurements of Electrochemically Active Surface Area... 20

Redox Wave Integration and Charge Reversibility ............................ 25 
Intrinsic Activity of Catalyst Films.............................................................. 32

Physical Characterization of Pulse and Continuously Deposited $\mathrm{Ni}(\mathrm{Fe})(\mathrm{OH})_{2}$

Conclusion and Bridge

III. DOMAIN STRUCTURES OF NI AND NIFE (OXY)HYDROXIDE OXYGENEVOLUTION CATALYSTS FROM X-RAY PAIR DISTRIBUTION FUNCTION ANALYSIS

Introduction

Experimental

Electrode Fabrication

Film Deposition

Film Conditioning

Ex Situ X-Ray Sample Preparation

High Energy X-ray Scattering Measurements and Pair Distribution Function (PDF) Analysis

Model PDF Calculations.

Results and Discussion

Modeling of $\mathrm{Ni}_{(1-z)} \mathrm{Fe}_{z} \mathrm{O}_{x} \mathrm{H}_{y}$ Structures. ..................................................... 46

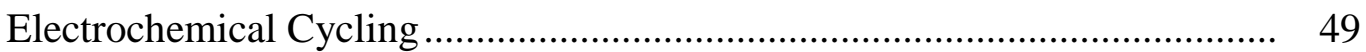

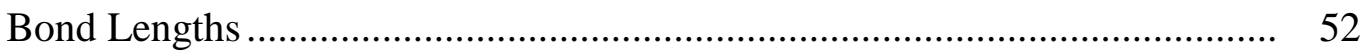

Effects of Pulsed Deposition on Morphology................................................ 54

Film Heterogeneity ……………………............................................... 57

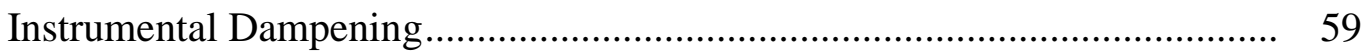

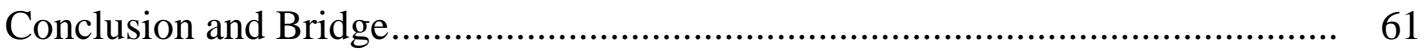


IV. OPTIMIZATION OF PULSED ELECTRODEPOSITIONS FOR HIGH PERFORMING NIFEO $\mathrm{X}_{\mathrm{Y}}$ FILMS _................................................................... 62

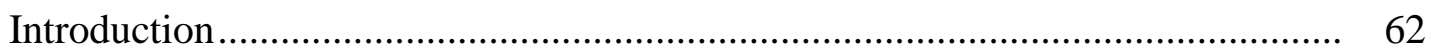

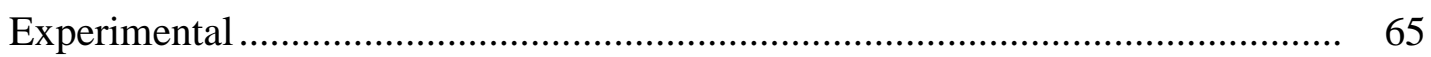

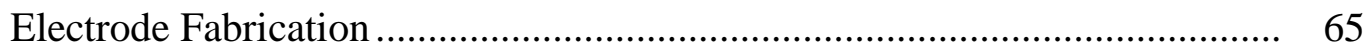

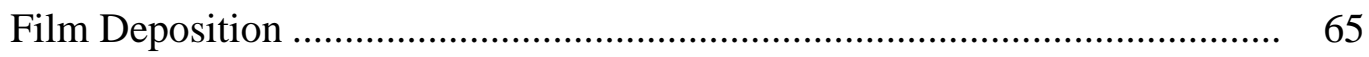

Electrochemical Measurements .............................................................. 66

Film Loading and TOF Calculcations..................................................... 66

Double Layer Capacitance ....................................................................... 67

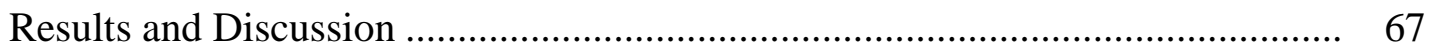

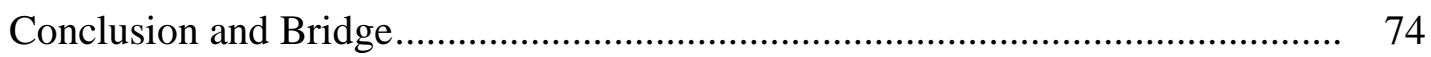

V. IN SITU CHARACTERIZATION OF THE OPTICAL PROPERTIES OF ELECTROCATALYSTS ............................................................................. 76

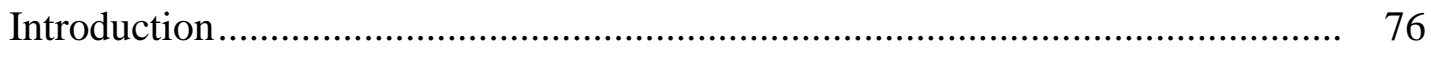

Electrochromism in Catalyst Films................................................................. 77

In Situ UV/Vis Absorption and Reflection Spectroscopy ................................... 78

Identification and Optimization of Catalyst Films for Solar Water Splitting Photoanodes ............................................................................................... 84

Tailoring of Catalyst Optical Properties ............................................................ 88

New Horizons for In Situ Optical Spectroscopy for Solar Fuels Material Characterization ………................................................................................ 89

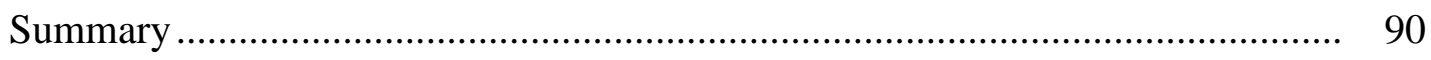

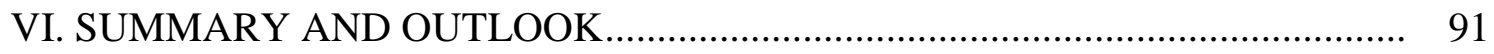




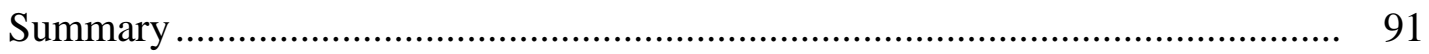

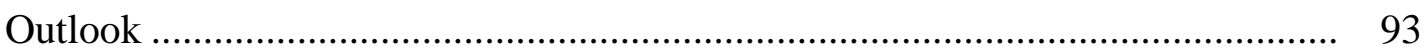

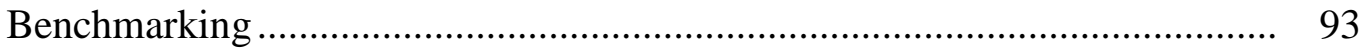

In Situ Techniques ....................................................................... 95

Beyond Pourbaix ........................................................................... 96

Deposition Mechanisms and Film Architecture ...................................... 96

Becoming True Material Scientists and Engineers ................................. 97

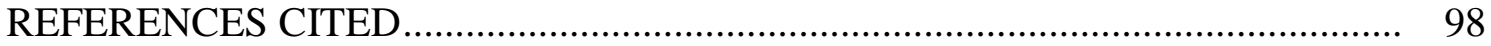




\section{LIST OF FIGURES}

Figure

Page

1.1 Schematic representation of the four commonly accepted phases of Ni-based catalysts in basic conditions. On the left, the transformation of the $\mathrm{Ni}$ hydroxide unit cell to a Ni sheet is shown, with hydrogens omitted for clarity. Transformations between phases are due to thermodynamic driving forces that depend on synthesis and electrochemical conditioning. The phases differ in degree of protonation, sheet spacing, sheet ordering, and extent of hydration......

2.1 Geometric current density for pulse and continuously deposited films as a function of overpotential ( $i R_{\mathrm{u}}$ corrected). Both films are electrodeposited from the same deposition solution of $0.05 \mathrm{M} \mathrm{FeCl}_{2}$ with $0.095 \mathrm{M} \mathrm{Ni}\left(\mathrm{NO}_{3}\right)_{2}$. As will be detailed below, the pulse-deposited films have an average $\mathrm{Fe}$ content of $\sim 15 \%$ that is higher than the continuous-deposited films $(12 \%<\mathrm{Fe}<\sim 7 \%)$ as well improved microstructure leading to enhanced activity..

2.2 Capacitance of continuously deposited $\mathrm{Ni}(\mathrm{Fe})$ (oxy)hydroxide films as a function of loading for a) uncharged films deposited on Au (dark and light blue) and FTO (dark and light red) electrodes as determined via cyclic voltammetry $\left(\mathrm{CV}\right.$, squares) and $\mathrm{AC}$ impedance spectroscopy $\left(Z_{\mathrm{AC}}\right.$, stars) with the data points at zero loading corresponding to the $C_{\mathrm{DL}}$ values of bare $\mathrm{Au}$ (aqua) and FTO (pink) electrodes and for b) charged films deposited on Au (blue) and FTO (red) electrodes as determined via impedance spectroscopy. The inset displays the equivalent circuit used to fit the impedance data. c) Voltammetry, and the corresponding double layer capacitance values determined via impedance spectroscopy as a function of potential for the five Au films of varying loading shown in $a$ and $b$

2.3 Voltammetry data for a continuously deposited $\mathrm{Ni}(\mathrm{Fe}) \mathrm{OOH}$ film with a thickness of $\sim 450$ MLE. The plot shows 1.5 cycles starting from a completely reduced film and then sweeping anodically. Any subsequent cycles would produce peaks that closely resemble those of the steady-state peaks.

2.4 Schematic representation of how changes in film conductivity could affect the magnitude of the steady-state $\mathrm{Ni}^{2+/ 3+}$ redox waves. During the oxidation sweep, the film becomes conductive as an oxyhydroxide and the entire film is oxidized. During the return reductive sweep, the film becomes electrical insulating as a hydroxide. Because the regions of the film nearest to the electrode surface are reduced first, this leaves regions of the films trapped in the oxidized state for the thicker films 
Figure

2.5 Comparison of loadings as determined by QCM and redox peak integration for both pulse (green) and continuously (blue) deposited films assuming a 1 e- per $\mathrm{Ni}$ oxidation and the appropriate amount of $\mathrm{Fe}$ (see experimental). The loading on both axes is given in monolayer equivalents (i.e. MLE) - one MLE is the number of metal ions needed to form a single $\mathrm{MOOH}$ monolayer on the electrode.

2.6 a) Loading-dependent reversibility (stars, dashed lines) and activity (squares, solid lines) of pulse (green) and continuously (blue) deposited films. $\chi_{\text {rev }}$ is defined in Eqn. 4. The data shown was collected on Au electrodes at $10 \mathrm{mV} \mathrm{s}^{-1}$. The activities were calculated from chronoamperometry data ( $\mathrm{iR}_{\mathrm{u}}$ corrected) at $300 \mathrm{mV}$ overpotential and normalized with a) total metal loadings determined using integration of the anodic wave in the first cycle, and b) total Fe loadings derived from Fe concentrations obtained through XPS. The lines are drawn to illustrate trends.

2.7 SEM Images of thick a) continuously and b) pulse-deposited films. Both top-down (i-ii) and cross-sectional (iii-iv) images are shown. The films were deposited at conditions that resulted in effective thicknesses of $~ 600 \mathrm{MLE}$ (continuous) and $~ 520$ MLE (pulsed).

3.1 a) Experimental and model PDF patterns of (top) NiOxHy and (bottom) $\mathrm{Ni}_{85} \mathrm{Fe}_{15} \mathrm{O}_{\mathrm{x}} \mathrm{H}_{\mathrm{y}}$ pulse-deposited films. Films shown were deposited and cycled once leaving them in a fully reduced state. The patterns (model and experimental) beyond $10 \AA$ have been scaled to give the high $\mathrm{r}$ peaks a similar intensity as the low $r$ peaks. In b), the residual function (i.e. goodness of fit) is plotted versus the number of unit cells contained in the model along the (100) and (010) directions. The models and unit cell are shown as insets. The $\mathrm{NiO}_{\mathrm{x}} \mathrm{H}_{\mathrm{y}}$ film matches well with a bilayer hydroxide domain. The $\mathrm{Ni}_{85} \mathrm{Fe}_{15} \mathrm{O}_{\mathrm{x}} \mathrm{H}_{\mathrm{y}}$ film is best represented by a smaller single-sheet domain.

3.2 PDF patterns of a) $\mathrm{NiO}_{\mathrm{x}} \mathrm{H}_{\mathrm{y}}, \mathrm{Ni}_{77} \mathrm{Fe}_{23} \mathrm{O}_{\mathrm{x}} \mathrm{H}_{\mathrm{y}}$, and b) $\mathrm{Ni}_{84} \mathrm{Fe}_{16} \mathrm{O}_{\mathrm{x}} \mathrm{H}_{\mathrm{y}}$ films in three different states of conditioning: as deposited (blue), oxidized (red), and reduced (cyan). "Residuals" of as-deposited minus re-reduced experimental patterns are shown to illustrate small structural changes upon cycling and are centered at $\mathrm{G}(\mathrm{r})=-0.5$. The optical absorption changes indicate that the as-deposited and reduced films contain $\mathrm{Ni}$ in the $2^{+}$oxidation state versus (nominally) $3^{+} / 4^{+}$in the oxidized films. The $\mathrm{NiO}_{x} \mathrm{H}_{y}$ and $\mathrm{Ni}_{77} \mathrm{Fe}_{23} \mathrm{O}_{\mathrm{x}} \mathrm{H}_{\mathrm{y}}$ systems in a) match well with a bilayer hydroxide and a single-sheet structure, respectively, both before and after cycling. In $b$, the as-deposited $\mathrm{Ni}_{84} \mathrm{Fe}_{16} \mathrm{O}_{\mathrm{x}} \mathrm{H}_{\mathrm{y}}$ film has a PDF pattern better fit by a bilayer structure, but the re-reduced film is better modeled as a single sheet 
3.3 M-O (bottom half) and M-O-M (top half) bond lengths for the films in nominally a) $\mathrm{Ni}^{2+}$ and b) $\mathrm{Ni}^{3+/ 4+}$ states, studied as a function of Fe content for continuous and pulse-deposited films. The squares/circles are for as-deposited and re-reduced films, respectively, while the triangles are for oxidized films. Each point represents only one sample, and therefore no error statistics are available.

3.4 Effect of the number of deposition pulses on the intermediate structure of $\mathrm{NiO}_{\mathrm{x}} \mathrm{H}_{\mathrm{y}}$ films. A) PDF patterns for catalysts deposited using 5, 10, 15, and 25 repeats of $2 \mathrm{~s}$ cathodic current pulses. B) Fit values as a function of domain dimension for each of the films shown in a). The patterns for films from 5 and 10 pulses are best fit by bi-layered structures while those with 15 and 25 pulses are best fit by single sheet structures. While the films synthesized with 15 and 25 pulses are best-fit by the same simulated structure, variations in their residual function show that they are not identical, and require a more-complex model for differentiation.

3.5 Heterogeneity in the $\mathrm{NiO}_{x} \mathrm{H}_{y}$ film with 5 pulses shown in Fig 4. Parameters varied include a) number of coherent layers in domain and b) domain dimension. Offset experimental and model PDF patterns (left) are shown with ternary diagrams (upper right) highlighting the optimal model for a linear combination of the three components. Overlaid PDF patterns of the experimental (grey) and optimized model blend (purple) are shown in the bottom right of both panels

3.6 PDF pattern of the $\mathrm{CeO}_{2}$ standard and the instrumental dampening function that will used to correct the model PDF patterns shown in this chapter.

4.1 Reversibility as a function of loading for four different deposition current densities. Each $0.2 \mathrm{~s}$ deposition pulse was followed by $30 \mathrm{~s}$ of stirring. The amount of material participating in the reaction was determined through both the ECSA (open squares) as measured through impedance spectroscopy as well as through QCM mass measurements (closed squares). The grey region, spanning nearly 500 MLEs, seems to represent a range of loading where the reversibility is maximized for the different systems. 
Figure

4.2 TOF at $300 \mathrm{mV}$ overpotential as a function of loading for four different deposition current densities. Each $0.2 \mathrm{~s}$ deposition pulse was followed by $30 \mathrm{~s}$ of stirring. The amount of material participating in the reaction was determined through the ECSA as measured through impedance spectroscopy. The grey region, spanning nearly 500 MLEs, seems to represent a range of loading where the TOF is maximized for the different systems. The data from ref 31 is included for comparison

4.3 TOF at $450 \mathrm{mV}$ overpotential as a function of loading for four different deposition current densities. Each $0.2 \mathrm{~s}$ deposition pulse was followed by $30 \mathrm{~s}$ of stirring. The amount of material participating in the reaction was determined through the ECSA as measured through impedance spectroscopy.....

4.4 Current as a function of loading for the films in Figs. 4.2 and 4.3. Each $0.2 \mathrm{~s}$ deposition pulse was followed by $30 \mathrm{~s}$ of stirring. The loading was determined from the ECSA as measured by impedance spectroscopy.

5.1 In situ electrochemical cells employed by a) Trotochaud et al. and b) Corrigan and Knight to collect optical spectroscopy data on catalysts films. In A), thin-film electrocatalysts were deposited by spin-coating onto the ITO substrate prior to electrode fabrication.

5.2 a) Cyclic voltammetry (CV) and $\mathrm{B})$ transmission spectra of $\mathrm{NiO}_{\mathrm{x}}$ film measured at potentials ranging from 0 to $0.6 \mathrm{~V}$ vs $\mathrm{Hg} / \mathrm{HgO}$ in $0.05 \mathrm{~V}$ increments. Each line in b) was taken at a potential corresponding to the dots on the $\mathrm{CV}$, with the top transmission line corresponding to the lowest potential.

5.3 Cross-sectional view showing the light path through an in situ liquid cell. The symbols a and $\mathrm{r}$ signify absorption and reflection probabilities, respectively, with the subscripts indicating the location of the optical event as described below. The spatial location of the arrows and symbols along the vertical scale of the diagrams is not significant..

5.4 a) Effective absorption coefficient $\alpha(\lambda)$ calculated for the active catalyst films.

b) Transmission probabilities $\operatorname{Tc}(\lambda)$ calculated for $\mathrm{Ni}_{0.9} \mathrm{Fe}_{0.1} \mathrm{O}_{\mathrm{x}}$ films of varying thickness.

5.5 Optical efficiency $\varphi_{\text {opt }}(\mathrm{t})$ of active catalyst films as a function of thickness $t$, assuming an optical band gap $1.8 \mathrm{eV}$ for the absorbing semiconductor and AM 1.5 solar flux. 
Figure

Page

5.6 a) Photoelectrode current voltage curves for $\mathrm{Ni}_{0.9} \mathrm{Fe}_{0.1} \mathrm{O}_{\mathrm{x}}$ and b) Optocatalytic efficiency $\Phi_{\mathrm{O}-\mathrm{C}}$ as a function of thickness $\mathrm{t}$ for different catalysts, assuming an optical band gap $1.8 \mathrm{eV}$ for the absorbing semiconductor and AM 1.5 solar flux.......

6.1 Cartoon highlighting factors that complicate the measurement of a true intrinsic TOF and their relative effective range. 


\section{CHAPTER I}

\section{INTRODUCTION}

\section{Challenges to Efficient Water Splitting}

The generation of hydrogen gas through water splitting has the potential to solve two significant renewable energy challenges. The first is mitigating the intermittency problem which arises from the variable nature of solar and wind based energy sources. ${ }^{1-3}$ The second is the minimization or elimination of carbon-based fuel sources as a $\mathrm{H}_{2}$ feedstock. $^{4,5}$

The base component of any water splitting system is an electrolyzer. The electrolyzer facilitates the oxygen and hydrogen evolution half reactions, OER and HER, respectively.

$$
\begin{aligned}
& 4 \mathrm{OH}^{-} \rightarrow 2 \mathrm{H}_{2} \mathrm{O}+\mathrm{O}_{2}+4 \mathrm{e}-(\text { OER in base }) \\
& 4 \mathrm{H}_{2} \mathrm{O}+4 \mathrm{e}-\rightarrow 2 \mathrm{H}_{2}+4 \mathrm{OH}^{-} \text {(HER in base) }
\end{aligned}
$$

Currently, the OER is a significant source of inefficiency in the overall water splitting process, ${ }^{6,7}$ and has thus received significant attention. To minimize the overpotential required to drive the OER, catalysts which can bring the activation energy of the four electron process on par with that of the HER are sought.

There are two main categories of water splitting devices being investigated today. The first, previously mentioned, is the stand-alone electrolyzer that takes as an input electricity from either renewable or conventional energy sources. ${ }^{8,9}$ The second is a 
photoelectrochemical cell (PEC) which combines, in a single device, a photovoltaic system with an electrolyzer. ${ }^{10,11}$ The important difference between the two systems is that they operate at different extremes of catalyst thickness. The electrolyzer requires high catalyst loading in order to minimize the overpotential applied, while the PEC uses a low catalyst loading due to both the potential for parasitic light absorption from catalysts, ${ }^{12,13}$ as well as the need for current matching between the anodic and cathodic electrocatalysts. $^{14}$

The overpotential $\eta$ for the both the OER and HER comes is three forms

$$
\eta_{\mathrm{tot}}=\eta_{\mathrm{act}}+\eta_{\mathrm{mt}}+\eta_{\mathrm{ct}}
$$

where $\eta_{\text {tot }}$ is the total overpotential, $\eta_{\text {act }}$ is the overpotential associated with chemical activation, and $\eta_{\mathrm{mt}}$ and $\eta_{\mathrm{ct}}$ are the overpotentials required to drive mass and charge transport through the film, respectively. In PECs, the low catalyst loadings minimize the transport contributions to overpotential, and the ideal catalyst properties balance chemical activation and transparency. ${ }^{12,13}$ However, for high loading films, such as those used in electrolyzers, mass and charge transport could play an increasing role as the amount of catalyst deposited is increased.

To drive down the cost of water splitting catalysts, ${ }^{15}$ earth-abundant transitionmetal-based catalysts that can oxidize water as effectively as platinum group metals (PGM) are being pursued. ${ }^{16,17}$ The use of first row transition metals has the drawback of film instability in acidic conditions. In alkaline conditions, Fe-doped $\mathrm{Ni}$ (oxy)hydroxide catalysts have shown superior performance to their PGM counterparts, ${ }^{18,19}$ and will be the focus of the entirety of this work. 
To overcome the low activity of monometallic transition metal systems, binary, ternary and quaternary metallic systems have been investigated. ${ }^{20,21} \mathrm{Co}$ and Ni-based systems doped with Fe have shown significant improvements, which has fueled speculation that $\mathrm{Fe}$ is the active site in these materials. ${ }^{22,23}$ The low activity of Fe-based systems could be due to its low conductivity at low overpotentials. ${ }^{24}$ However, the widely speculated participation of lattice oxygen in the redox reaction, ${ }^{25,26}$ cautions the assignment of any one species as the active site, and points toward abandoning a metalcentric focus which ignores the solid state nature of the energy distributions within the transition metal oxide materials.

\section{Synthesis and Structure of Ni-based OER Catalysts}

$\mathrm{NiO}_{\mathrm{x}} \mathrm{H}_{\mathrm{y}}$ materials can be synthesized through a wide variety of techniques.

Hydroxides are typically made through cathodic electrodeposition, ${ }^{27,28}$ while oxides are generated when heat treatments are required such as with sol-gel, ${ }^{29}$ solvo- and hydrothermal processes ${ }^{30-32}$. Recently, Ni-based catalysts with non-oxygen anions have been created to include selenides, ${ }^{33}$ phosphides ${ }^{34}$ and sulfides, ${ }^{35}$ but the so-called borates $^{36}$ do not actually contain those anions within the lattice.

The most thermodynamically stable phases of the elements are highlighted in Pourbaix diagrams, ${ }^{37}$ which display the predominant species at a given $\mathrm{pH}$ and electrical potential. For Ni-based catalysts within the stability limits of water, hydroxide phases dominate at high $\mathrm{pH}$. As the films are oxidized, an nominally oxyhydroxide phase is formed. This process is represented by the Bode diagram ${ }^{38}$ shown in Figure 1.1 

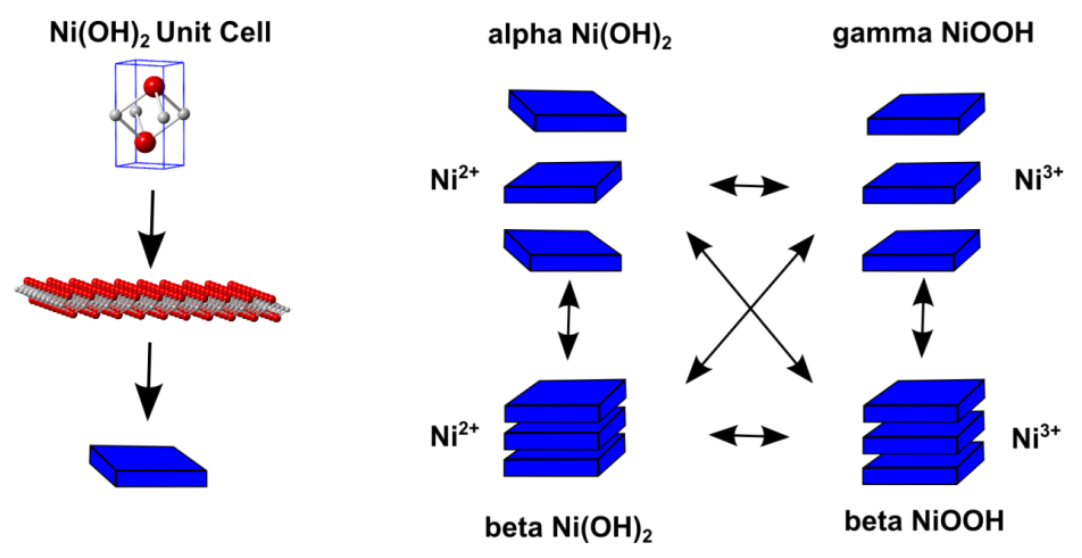

Figure 1.1. Schematic representation of the four commonly accepted phases of Ni-based catalysts in basic conditions. On the left, the transformation of the Ni hydroxide unit cell to a Ni sheet is shown, with hydrogens omitted for clarity. Transformations between phases are due to thermodynamic driving forces that depend on synthesis and electrochemical conditioning. The phases differ in degree of protonation, sheet spacing, sheet ordering, and extent of hydration.

The alpha hydroxide phase is characterized by disordered stacking of hydroxide sheets with interlayer water and ions present. Oxidation of the alpha phase produces a gamma phase that maintains the interlayer species. Several works studying these transitions have been published, those only recently in Fe-free electrolytes has it been shown that overcharging, aging, and extended cycling likely lead to the formation of the more thermodynamically favored, dehydrated, beta phases. ${ }^{39-43}$ Other important structural considerations are the presence of multiple phases and non-stoichiometry in the films. ${ }^{44}$ Depending on the synthetic technique and degree of transition metal doping, the appearance of phase segregated or crystalline domains have been demonstrated. ${ }^{23,45,46}$

Despite being highlighted nearly 30 years ago, ${ }^{47}$ it is only recently that the field of OER catalysis has recognized the importance of Fe introduction, whether intentional or as an impurity in commercially procured electrolyte, in catalyzing the OER. ${ }^{24,40,48} \mathrm{~A}$ broad range of $\mathrm{Ni}: \mathrm{Fe}$ stoichiometries have been reported to be the most active. ${ }^{40,45,48-50}$ Beyond enhancing the catalytic activity, significant structural changes have also been 
reported following Fe doping to include changes in both lengths ${ }^{23,51}$ and expanded sheet spacing. ${ }^{40,52}$

While the facile electrodeposition process is beneficial for generating the more thermodynamically stable phase (i.e., hydroxides in alkaline solutions), it suffers from the preferential deposition of certain metals in amounts that differ from their solution

concentration. ${ }^{53}$ Other solution based methods enable a more reliable film stoichiometry, but suffer from the generation of non-alkaline-stable oxide phases during high temperature processing, which gradually convert to (oxy)hydroxides. ${ }^{19}$

\section{Characterization of Disordered Films}

Ni-based OER catalysts are often amorphous materials, ${ }^{50,54,55}$ which has hindered their study via traditional crystallographic techniques. Acquiring detailed structural information from laboratory scale (low energy) x-ray diffraction relies on the presence of periodic planes of atoms. The greater the extent of crystallinity in a material, the sharper (and more numerous) the peaks become, and the more morphological details that can be extracted. However, the low energy and intensity of laboratory x-ray sources limit the effectiveness of this technique for highly disordered systems. ${ }^{56}$

High energy synchrotron sources allow for the collection of diffuse (non-Bragg) scattering information from amorphous samples that would normally be discarded as background in laboratory scale XRD instruments. ${ }^{57}$ The Fourier transform of this total scattering data can provide real space atom-atom correlations that are not achievable using traditional crystallography. The drawback of looking at the real space data from 
high energy sources is that turbostratic disorder between layers of atoms results in the loss of information due to randomness in the atom pair correlations. ${ }^{58}$

An alternative to scattering techniques, $\mathrm{x}$-ray absorption spectroscopy has the advantage of provide element specific information, due to the unique absorption signatures of the different transition metals. Several of the high performing systems have been investigated, providing insight into metal oxygen and metal-metal bond distances. ${ }^{59,60}$ The limitation to these techniques is that only the first two or three coordination spheres can be probed, so details of nanoscale dimensions are not accessible. Particle sizes can be estimated by decay in the pair distribution function, ${ }^{61,62}$ or through the use of the Scherrer equation in $\mathrm{XRD}^{31,63}$. However, limited q resolution and experimental setup can introduce ambiguity into the length of scattering coherence. ${ }^{64}$ The use of electron microscopy techniques can help fill in the gaps in the previous scattering techniques by providing images of the material. ${ }^{65,66}$

\section{Summary and Bridge}

Despite its ubiquity in battery materials, and long history in OER, the structureactivity relationships of Ni hydroxides are not well understood. Early (and unfortunately contemporary) work on both structure and activity must be evaluated cautiously, due to the unintended incorporation of Fe into the Ni films, which often goes unquantified. A wide range of architectures and synthetic techniques have been reported. A key knowledge gap is how these different syntheses and architectures impact performance.

Chapter II addresses this knowledge gap by connecting the structure and activity of films created via two different techniques: continuous and pulsed electrodepositions. 
A physical interpretation of the loading dependent activity is provided, including a comparison of commonly reported ways of calculating the electrochemically active surface area. An improvement in the transport properties of the film is shown to enhance the catalytic activity of the $\mathrm{NiFeO}_{\mathrm{x}} \mathrm{H}_{\mathrm{y}}$ films, even at loadings approaching $100 \mu \mathrm{g} \mathrm{cm}^{-2}$.

This chapter contains no previously published or coauthored material. Portions of Chapter II were published as Batchellor, A. S.; Boettcher, S. W. Pulse-Electrodeposited $\mathrm{Ni}-\mathrm{Fe}$ (Oxy)hydroxide Oxygen Evolution Electrocatalysts with High Geometric and Intrinsic Activities at Large Mass Loadings. ACS Catal. 2015, 5 (11), 6680-6689. A.S.B. conducted all of the experiments and wrote the manuscript. S.W.B. was the principal investigator and provided editorial assistance. Portions of Chapter III were published Batchellor, A.S.; Kwon, G.; Tiede, D. M.; Boettcher, S. W. Domain Structures of Ni and NiFe (Oxy)Hydroxide Oxygen-Evolution Catalysts from X-ray Pair Distribution Function Analysis. J. Phys. Chem. C. 2016, Submitted. A.S.B. processed and analyzed all of the raw data and wrote the manuscript. S.W.B. was the principal investigator and provided editorial assistance. D.M.T. and G.K collected the raw data and provided training on PDF analysis techniques. Chapter IV contains no previously published or coauthored material. Portions of Chapter V were published as Batchellor, A.S.;

Trotochaud, L.; Boettcher, S. W. In Situ Characterization of the Optical Properties of Electrocatalysts, In Advanced and In Situ Analytical Methods for Solar Fuel Materials 2015, Volume 371 of the series Topics in Current Chemistry, pp 253-324. A.S.B. wrote the manuscript. L.T., having performed much of the cited experimental work, provided insights and clarifications. S.W.B. was the principal investigator and provided editorial assistance. Chapter VI contains no previously published or coauthored material. 


\section{CHAPTER II}

\section{PULSE-ELECTRODEPOSITED NI-FE (OXY)HYDROXIDE OXYGEN EVOLUTION ELECTROCATALYSTS WITH HIGH GEOMETRIC AND INTRINSIC ACTIVITIES AT LARGE MASS LOADINGS}

Portions of this chapter were previously published as Batchellor, A. S.; Boettcher, S. W. Pulse-Electrodeposited Ni-Fe (Oxy)hydroxide Oxygen Evolution Electrocatalysts with High Geometric and Intrinsic Activities at Large Mass Loadings. ACS Catal. 2015, 5 (11), 6680-6689. A.S.B. conducted all of the experiments and wrote the manuscript. S.W.B. was the principal investigator and provided editorial assistance.

\section{Introduction}

The overpotential associated with the oxygen evolution reaction (OER) limits the efficiency of hydrogen production by water electrolysis. ${ }^{1-3}$ Despite significant effort devoted to catalyst discovery, ${ }^{4-6}$ improvements in activity have not emerged for several decades and OER catalysts are substantially worse at oxidizing water than, for example,

Pt is at reducing it. ${ }^{4,5,7,8}$ Practically, OER catalysts need to operate at current densities of $\sim 0.5$ and $2 \mathrm{~A} \mathrm{~cm}^{-2}$, in either alkaline or proton-exchange membrane electrolyzers, respectively. ${ }^{9}$ Barring the discovery of new catalysts with several-order-of-magnitude improvement in activity, high mass loading is the only means of achieving these current densities at moderate overpotentials (i.e. near $300 \mathrm{mV}$ ). 
One relevant catalyst performance metric (in addition to cost and stability) is the overpotential required to achieve a certain geometric current density. The total overpotential $\eta_{\text {tot }}$ required to drive the reaction at a given current density can be described by

$$
\eta_{\mathrm{tot}}=E_{\mathrm{app}}-E^{\mathrm{o}}=\eta_{\mathrm{act}}+i R_{\mathrm{film}}+i R_{\mathrm{mt}}+i R_{\mathrm{u}}
$$

where $E_{\text {app }}$ is the potential applied by the potentiostat, $E^{o}$ is the thermodynamic equilibrium potential of the OER, $i$ is the current, $\eta_{\text {act }}$ is the kinetic overpotential associated with overcoming the activation energy barrier of the reaction, $i R_{\text {film }}$ is the voltage drop associated with the resistance felt by electrons traveling through the film, $i R_{\mathrm{mt}}$ is the voltage drop required to drive mass transport of ions in, and $\mathrm{O}_{2}$ out, of the catalyst layer, and $i R_{\mathrm{u}}$ is the uncompensated series resistance of the solution and wires. The values of $i R_{\mathrm{film}}$ and $i R_{\mathrm{mt}}$ can vary with position in a three-dimensional catalyst material. Because $i R_{\mathrm{u}}$ depends on cell engineering, and is largely independent of the catalyst film, we ignore it in our discussion of catalyst structure-activity relationships.

There are three routes to minimize $\eta_{\text {tot }}$. The first is by lowering of $\eta_{\text {act }}$, which is specific to the chemistry of the active site. This could be achieved by discovering higheractivity sites, e.g., through experimental or computational screening. Second, increasing mass loading can lower $\eta_{\text {tot }}$ if the added mass increases the number of electrochemically accessible active sites (such that the new mass provides parallel reaction paths through increases in the electrolyte-catalyst interface area) and is done in a way that minimizes increases in $i R_{\mathrm{film}}$ or $i R_{\mathrm{mt}}$. The performance of catalyst films prepared with high mass loadings to reach current densities relevant to industrial electrolyzers has been reported for both Ni-based ${ }^{10}$ and $\mathrm{Ni}(\mathrm{Fe})$-based systems. ${ }^{11}$ Finally, the losses associated with 
electron and mass transport, $i R_{\mathrm{film}}$ or $i R_{\mathrm{mt}}$, could themselves be reduced by micro- and nanoscale engineering of the film architecture. The minimization of $i R_{\text {film }}$ could adversely affect $i R_{\mathrm{mt}}$ as the former would benefit from a dense film (lower electrical resistivity), while the latter from an open, porous structure (faster mass transport). The system is further complicated by the reality that the electrical and mass-transfer resistance at each active site is a function of its position in the film. The interconnected porous structure also complicates modeling of catalyst utilization due to bubble nucleation and mass transport effects. ${ }^{12}$

Several routes to designing architectures with good electrical connectivity and high surface area have been explored. Lu et al. and Perez-Alonso et al. showed high OER current densities by depositing $\mathrm{Ni}(\mathrm{Fe}) \mathrm{OOH}$ into a porous $\mathrm{Ni}$ foam. ${ }^{13,14}$ Ma et al. stacked alternating layers of $\mathrm{Ni}(\mathrm{Fe}) \mathrm{OOH}$ and conductive reduced graphene oxide. ${ }^{15}$ Gong et al. reported higher electrocatalytic activity by using carbon nanotubes as scaffolds for $\mathrm{Ni}(\mathrm{Fe}) \mathrm{OOH} .{ }^{16}$ Marini et al. used selective dissolution of $\mathrm{Al}$ to form a porous "Raney" Ni OER catalyst. ${ }^{17}$ Gao et. al. synthesized $\alpha-\mathrm{Ni}(\mathrm{OH})_{2}$ hollow nanospheres which had relatively high OER activity after activation. ${ }^{18}$ Hunter et al. optimized a pulsed laser ablation technique, generating $\mathrm{Ni}(\mathrm{Fe}) \mathrm{O}_{x}$ nanoparticles that were solution cast onto highly ordered pyrolytic graphite. ${ }^{19}$

For many transition metal-based OER catalysts in neutral to basic conditions the "porosity" (or electrochemically active surface area) extends to the molecular scale. Doyle and coworkers, for example, discuss OER catalysis in hydrated phases on the surface of metal and oxide electrodes. ${ }^{20}$ Trotochaud et al. showed that nanocrystalline $\mathrm{NiO}_{x}$ thin films convert to $\mathrm{Ni}(\mathrm{OH})_{2} / \mathrm{NiOOH}$ (while absorbing Fe impurities from the 
electrolyte) where each $\mathrm{Ni}$ appears redox active and thus in direct contact with the electrolyte. ${ }^{21}$ Klingan et al. studied amorphous $\mathrm{CoO}_{x}$-based catalysts and demonstrated activity throughout the bulk of the disordered, electrolyte-permeable material, which was termed "volume" catalysis. ${ }^{22}$ May et al. examined the interplay of activity and a changing surface structure on various oxide perovskites as they evolved from crystalline states under OER conditions, and concluded that for the perovskites investigated, the chemical composition that underwent the greatest surface amorphization (and hydration) resulted in the greatest OER activities. ${ }^{23}$ In the $\mathrm{Ni}(\mathrm{Fe}) \mathrm{OOH}$ materials studied here, the different structures present under OER and non-OER conditions are typically represented in the well-known Bode scheme. ${ }^{24}$ From previous work, ${ }^{7}$ the as-deposited material likely adopts a disordered $\alpha-\mathrm{Ni}(\mathrm{OH})_{2} / \gamma-\mathrm{NiOOH}$ structure with a somewhat expanded intersheet spacing due to the presence of $\mathrm{Fe}^{3+}$ substituting on $\mathrm{Ni}^{2+}$ sites and the intercalation of charge-compensation ions and water. ${ }^{25,26}$

In order to optimize performance, it is also important to assess the intrinsic catalyst activity as a function of morphology and loading, for example normalized to the number of active sites. A simple way to estimate "intrinsic" activity is by normalizing the rate of product production to the total number of electrochemically accessible metal ions to obtain an effective "total-metal" turnover frequency $\left(\mathrm{TOF}_{\mathrm{tm}}\right)$ :

$$
\mathrm{TOF}_{\mathrm{tm}}=\mathrm{mol} \mathrm{O}_{2} \text { generated } \cdot(\text { mol total metal })^{-1} \cdot \mathrm{s}^{-1}
$$

$\mathrm{TOF}_{\mathrm{tm}}$ is a useful metric for comparing the activities of different catalyst compositions because it does not rely on identification of a specific active site and can be applied to any system. ${ }^{27}$ Recent work, however, suggests that $\mathrm{Fe}$ is the active site in $\mathrm{Ni}(\mathrm{Fe}) \mathrm{OOH},{ }^{25,28}$ and we have previously calculated an apparent TOF based only on Fe 
content to support our conclusion that $\mathrm{Fe}$ is likely the active site in $\mathrm{Co}(\mathrm{Fe}) \mathrm{OOH}$ also. ${ }^{29} \mathrm{In}$ this work we therefore also report the apparent intrinsic activity normalized only to the number of Fe cations $\left(\mathrm{TOF}_{\mathrm{Fe}}^{\mathrm{app}}\right)$ :

$$
\mathrm{TOF}_{\mathrm{Fe}}^{\mathrm{app}}=\mathrm{mol} \mathrm{O}_{2} \text { generated } \cdot(\text { mol total } \mathrm{Fe})^{-1} \cdot \mathrm{s}^{-1}
$$

$\mathrm{TOF}_{\mathrm{Fe}}^{\mathrm{app}}$ is a useful metric for determining whether or not changes in activity can be connected to changes in Fe concentration or to other phenomena. We note, however, that in the case of $\mathrm{Ni}(\mathrm{Fe}) \mathrm{OOH}$, the $\mathrm{NiOOH}$ is a critical component to the catalyst, serving to both electrically wire the Fe sites to the conductive electrode and to chemically stabilize them to dissolution. ${ }^{30}$ There are also strong electronic interactions between the cations, and the minimum structural unit required for the catalytic active site remains unclear.

Under ideal conditions where electronic and mass transfer resistances are negligible (e.g. for very thin films supported on a conductive electrode), ${ }^{21,29}$ a reasonable estimate of the intrinsic $\mathrm{TOF}_{\mathrm{tm}}$ can be measured. For high-surface-area thick films, however, TOF $\mathrm{tm}_{\mathrm{m}}$ data extracted using Eqn. 2 could include the effects of $R_{\mathrm{film}}$ or $R_{\mathrm{mt}}$. Understanding the interplay in how the measured $\mathrm{TOF}_{\mathrm{tm}}$ is modulated by film thickness is therefore expected to provide insight into the role of activation, mass transport, and electrical film resistance on the overall measured performance.

The importance of thickness-dependent effects has been highlighted in several recent reports. In the thin-film limit, Yeo and Bell showed that substrate choice modulates the activity of the first few monolayers of both $\mathrm{CoO}_{x}$ and $\mathrm{Ni}(\mathrm{Fe}) \mathrm{O}_{x}$ films. ${ }^{31,32}$ For thicker films under near-neutral conditions, Bediako et al. found that the electrochemical reaction rate was controlled by both the diffusion of protons in the film 
and the rate of the catalytic reaction. ${ }^{33}$ Similar effects associated with buffer-mediated proton diffusion were observed by Klingan et al. ${ }^{22}$

Herein we seek to connect fundamental "intrinsic" activities to practical performance as a function of film loading. Using the $\mathrm{Ni}(\mathrm{Fe}) \mathrm{OOH}$ system, which is the fastest known OER catalyst in base, ${ }^{7,27,34}$ we quantify loading and electrochemical activity using impedance measurements of capacitance, quartz-crystal-microbalance (QCM) measurements of mass, and integration of the Ni redox features. We compare the results from these measurements and discuss methods for estimating intrinsic activity as a function of loading. We report a pulsed-electrodeposition technique that (1) eliminates the decrease in the effective activity of each metal site with increased mass loading resulting from a continuous deposition, (2) creates a more-homogenous Fe distribution in the film, (3) improves the connectivity of the films as demonstrated through improved film electrochemical reversibility, and (4) produces films whose performance is comparable to films with advanced architectures, for example passing a current density of $120 \mathrm{~mA} \mathrm{~cm}^{-2}$ at $300 \mathrm{mV}$ overpotential in $1 \mathrm{M} \mathrm{KOH}$ at room temperature with a loading of $730 \mathrm{nmol} \mathrm{metal} \mathrm{cm}^{-2}$.

\section{Experimental}

\section{Electrode Fabrication.}

Conductive Au-coated electrode substrates were prepared by first cleaning glass slides in a 3:1 hydrogen peroxide to sulfuric acid (piranha) solution followed by plasma etching. Next, $10 \mathrm{~nm}$ of Ti and $100 \mathrm{~nm}$ of Au were deposited by electron beam 
evaporation. Both fluorine-doped tin oxide (FTO) and Au/Ti coated glass slides were cut into $\sim 0.75-1.0 \mathrm{~cm}^{2}$ squares. $\mathrm{Cu}$ wire was attached to the slides via $\mathrm{Ag}$ paint. The entire perimeter of the FTO slides was covered in Ag paint to reduce series resistance. Exposed wire and Ag paint were sealed with epoxy (Hysol 1C). Au QCM slides were purchased from Stanford Research Systems.

\section{Film Deposition.}

Prior to film deposition, the Au and FTO electrodes were cycled twice in $1 \mathrm{M}$ $\mathrm{KOH}$ from 0.0 to $0.65 \mathrm{~V}$ vs. $\mathrm{Hg} / \mathrm{HgO}$. The deposition cell used a two electrode configuration with carbon cloth serving as the counter electrode. The films were cathodically deposited from a $0.1 \mathrm{M}$ solution prepared by mixing $\mathrm{Ni}\left(\mathrm{NO}_{3}\right)_{2} \bullet 6 \mathrm{H}_{2} \mathrm{O}$ (Alfa Aesar) and $\mathrm{FeCl}_{2} \bullet 4 \mathrm{H}_{2} \mathrm{O}$ (Sigma Aldrich) in a 95:5 (mol:mol) ratio with ultrapure 18.2 $\mathrm{M} \Omega \mathrm{cm}$ water as the solvent. The deposition solution was sparged with $\mathrm{N}_{2}$ for 20 min prior to film deposition. All films were deposited at a cathodic current density of -10 mA $\mathrm{cm}^{-2}$. Continuously deposited films were subjected to reducing potentials for times ranging from 3 to $20 \mathrm{~s}$. Pulse-deposited films were subjected to sequential $2 \mathrm{~s}$ cathodic current pulses at $-10 \mathrm{~mA} \mathrm{~cm}{ }^{-2}$. Following each pulse, the solution was stirred for $10 \mathrm{~s}$ to ensure homogeneity of the deposition solution prior to the next pulse. Between 2 and 20 cycles were used to achieve a range of loadings similar to those obtained using the continuous deposition. All films were briefly dipped into ultrapure water prior to being submerged in the electrochemical cell to remove residual metal ions. 


\section{Electrochemical Measurements.}

The electrochemical cell was set up in a three electrode configuration with a $\mathrm{Pt}$ counter electrode and a $1 \mathrm{M} \mathrm{KOH} \mathrm{Hg/HgO} \mathrm{reference} \mathrm{electrode.} \mathrm{The} \mathrm{polypropylene} \mathrm{cell}$ contained a 1.0 M solution of semiconductor-grade KOH (Sigma Aldrich) prepared with 18.2 $\mathrm{M} \Omega \mathrm{cm}$ water. The electrochemical cell was sparged continuously with $\mathrm{O}_{2}$ and magnetically stirred to dislodge bubbles on the electrode surface. Measurements were taken on a Biologic SP-200 potentiostat. Electrochemical data were corrected for the uncompensated series resistance $R_{\mathrm{u}}$, which was determined through fitting of AC impedance data to a modified Randles circuit. The range of $R_{\mathrm{u}}$ was 4-6 $\Omega$. The overpotential was determined by $\eta=E_{\mathrm{app}}-E^{\mathrm{o}}-i R_{\mathrm{u}}$.

\section{Film Loading and TOF Calculations.}

All film loadings reported were obtained through the integration of the (nominally) $\mathrm{Ni}^{2+/ 3+}$ anodic redox peak of the fully reduced film. To avoid any irregularities associated with the freshly deposited film, two CV cycles were used to equilibrate the film with the solution in the electrochemical cell prior to taking any electrochemical measurements. The presence of oxidized Ni ions after the completion of a reducing sweep was readily apparent from a dark color on the electrode surface, compared to the light green/transparent fully reduced film. ${ }^{35}$ To return films to their fully reduced initial state, the partially oxidized films were held at reducing potentials (up to $1.0 \mathrm{~V}$ vs $\mathrm{Hg} / \mathrm{HgO}$ ) until all the dark color associated with the oxidized films disappeared ( $<20 \mathrm{~min}$ for the thickest films). No large cathodic reduction waves were observed which suggests that Ni ions were not reduced to metallic Ni during this process. 
$\mathrm{Ni}$ loadings were calculated by integrating the $\mathrm{Ni}^{2+/ 3+}$ oxidation wave, assuming one electron per Ni oxidized. Total loadings for pulse-deposited films were obtained by dividing by 0.85 to reflect the roughly $15 \%$ Fe incorporated into each film that does not contribute to the integrated redox wave. This Fe content was determined by x-ray photoelectron spectroscopy (XPS) analysis, as described below. Total loadings for continuously deposited films were obtained in the same way, except the Fe concentration was calculated by assuming a gradient in $\mathrm{Fe}$ with the amount of $\mathrm{Fe}$ in the outer surface sampled by XPS varying linearly from $12 \%$ to $4 \%$ between the highest and lowest loading films, respectively. The approximation of one electron per Ni was confirmed QCM mass measurements for a range of film thicknesses (see below). These loadings are reported in units of $\mathrm{nmol} \mathrm{cm}$ as well as in monolayer equivalents (MLE), which are mathematically equivalent to a surface roughness factor assuming the layered $\mathrm{MOOH}$ structure. Given the molecular weight of $\mathrm{Ni}(\mathrm{Fe}) \mathrm{OOH}$ of $\sim 92 \mathrm{~g} / \mathrm{mol}$, the largest loadings of $\sim 1000 \mathrm{nmol} \mathrm{cm} \mathrm{cm}^{-2}$ correspond to $\sim 100 \mu \mathrm{g} \mathrm{cm}^{-2}$. All TOF data were acquired through chronoamperometry with an $i R_{\mathrm{u}}$ correction applied and the number of metal sites taken from the loading as described above.

\section{Double Layer Capacitance.}

Capacitance values obtained from voltammetry were acquired over the range of 0.1 to $0.0 \mathrm{~V}$ and 0.0 to $0.1 \mathrm{~V}$ vs. $\mathrm{Hg} / \mathrm{HgO}$ on FTO and $\mathrm{Au}$ electrodes, respectively. The more-anodic potential range on the Au electrode was used to eliminate a small background current likely due to the oxygen reduction reaction on Au. Charging currents 
were measured at scan rates of 100, 200, 400 and $800 \mathrm{mV} \mathrm{s}^{-1}$. Capacitance was determined from the slopes obtained by plotting current vs. scan rate.

AC impedance measurements were taken over the frequency range of $0.1 \mathrm{~Hz}$ to $100 \mathrm{kHz}$. Impedance measurements on charged films were taken at $0.52 \mathrm{~V}$ vs. $\mathrm{Hg} / \mathrm{HgO}$ and on uncharged films at either $-0.05 \mathrm{~V}$ or $0.05 \mathrm{~V}$ vs. $\mathrm{Hg} / \mathrm{HgO}$ for FTO and $\mathrm{Au}$ electrodes, respectively. Capacitance values were then obtained through fitting (EC Lab) of the impedance spectrum using an equivalent circuit containing a resistor $\left(R_{\mathrm{u}}\right)$ connected in series to a resistor (charge transfer resistance $R_{\mathrm{CT}}$ ) and a capacitor (double layer capacitance $C_{\mathrm{DL}}$ ) that were connected in parallel.

\section{Film Characterization.}

Scanning electron microscopy (SEM) images were collected on a Zeiss Ultra 55 SEM at $5 \mathrm{kV}$. XPS studies were carried out on an ESCALAB 250 (ThermoScientific) using a $\mathrm{Mg} \mathrm{K} \alpha$ nonmonochromated flood source (200 W, $75 \mathrm{eV}$ pass energy). The samples were charge-neutralized using an in-lens electron source combined with a lowenergy $\mathrm{Ar}^{+}$flood source. Spectra were analyzed, including satellite subtraction for the nonmonochromated x-ray source, using ThermoScientific Avantage 4.75 software.

\section{Results and Discussion}

\section{Performance of Electrochemically Deposited Catalyst Films.}

We first contrast two different methods, continuous and pulsed electrodeposition from the same precursor solution, to prepare catalyst films with high loading on planar 
supports. Figure 2.1 shows that the performance of the pulse-electrodeposited films are superior to those of continuously electrodeposited films at all overpotentials.

The performance of the pulse-electrodeposited films on planar electrodes is also similar to previously reported advanced electrode architectures described in the introduction where $\mathrm{Ni}(\mathrm{Fe}) \mathrm{OOH}$ is often supported on conductive OER-inert scaffolds, such as carbon nanotubes or macroporous metallic Ni foam. ${ }^{13,15,16,19}$ The high activity of the pulse-electrodeposited films on planar supports suggests that further optimization of deposition conditions on Ni foam electrodes, for example, would lead to substantial improvements in performance. In the remaining portions of this manuscript we highlight differences in film morphology, Fe content, and electrochemical behavior arising from the two deposition techniques, and discuss the potential contributions of each to the behavior observed in Figure 2.1. This analysis provides insight into the design of highactivity OER catalyst architectures. 
a

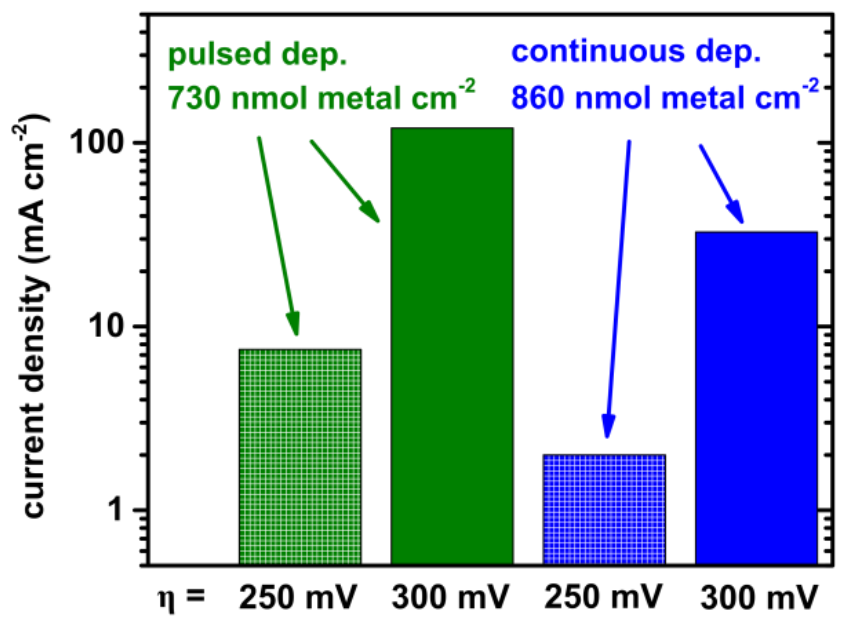

b

overpotential (V) - iR

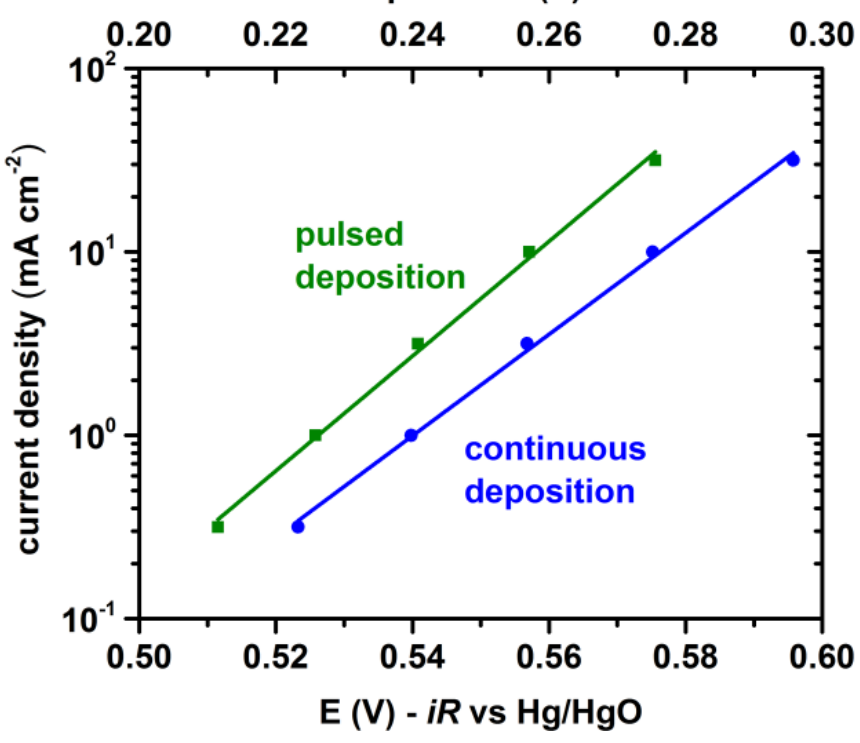

Figure 2.1. Geometric current density for pulse and continuously deposited films as a function of overpotential ( $i R_{\mathrm{u}}$ corrected). Both films are electrodeposited from the same deposition solution of $0.05 \mathrm{M} \mathrm{FeCl}_{2}$ with $0.095 \mathrm{M} \mathrm{Ni}\left(\mathrm{NO}_{3}\right)_{2}$. As will be detailed below, the pulse-deposited films have an average Fe content of $\sim 15 \%$ that is higher than the continuous-deposited films $(12 \%<\mathrm{Fe}<\sim 7 \%)$ as well improved microstructure leading to enhanced activity.

\section{Determining loading and effective TOF.}

While practical metrics (e.g. $\mathrm{A} / \mathrm{cm}^{2}$ ) are valuable for the identification of catalysts and supported catalysts that can achieve practically relevant current densities, they are 
limited in their ability to aid in the design of optimized catalyst films. The main reason for this is that they do not take into account the amount of material contributing to OER, and therefore do not describe intrinsic activity on a per-metal-ion basis.

The number of electrochemically active metal ion sites, and thus the intrinsic activity, has been estimated in several ways. McCrory et al. calculated surface roughness using the double layer capacitance $\left(C_{\mathrm{DL}}\right)$ of the films, as others have done. ${ }^{5,31,36}$ Corrigan integrated redox waves using cyclic voltammetry on $\mathrm{Ni}(\mathrm{OH})_{2} / \mathrm{NiOOH} \cdot{ }^{34}$ Klingan and Yeo used the charge passed during anodic electrodeposition to determine the number of metal ions deposited. ${ }^{22,32}$ Gravimetric methods have also been used, assuming all metals are electrochemically active. ${ }^{21,37}$ Here we explore the first two methods (double layer capacitance and redox wave integration) as a function of both loading and potential to determine under which experimental conditions each method may be accurately employed.

\section{Capacitance measurements of electrochemically active surface area.}

Figure 2.2a shows the $\mathrm{C}_{\mathrm{DL}}$ for $\mathrm{Ni}(\mathrm{Fe})$ (oxy)hydroxide films deposited on $\mathrm{Au}$ and FTO electrodes as determined through cyclic voltammetry (CV) and AC impedance following the benchmarking guidelines set forth by McCory et al. The measured $\mathrm{C}_{\mathrm{DL}}$, and hence apparent electrochemically active surface area (ECSA), are independent of loading in the uncharged state $(\sim 0.0 \mathrm{~V}$ vs $\mathrm{Hg} / \mathrm{HgO})$, and equivalent to the bare electrodes. The data also show that the $\mathrm{C}_{\mathrm{DL}}$ for the films are electrode dependent, with the $\mathrm{C}_{\mathrm{DL}}$ for $\mathrm{Au}$ substrates being a factor of four higher than on FTO. This is consistent with capacitance measurements on bare $\mathrm{Au}^{38}$ and $\mathrm{FTO},{ }^{39}$ and indicates that the capacitance of the 
electrode substrate, not that of the deposited $\mathrm{Ni}(\mathrm{Fe})(\mathrm{OH})_{2}$, is being measured. The cause of this behavior is the low conductivity $\left(\sim 10^{-6} \mathrm{~S} \mathrm{~cm}^{-1}\right)$ of the $\mathrm{Ni}(\mathrm{Fe})(\mathrm{OH})_{2}$ films in the potential region examined ( 0 to $0.1 \mathrm{~V}$ and 0 to $-0.1 \mathrm{~V}$ vs $\mathrm{Hg} / \mathrm{HgO}$ on $\mathrm{Au}$ and FTO

electrodes, respectively), ${ }^{7}$ and the electrolyte permeability of the catalyst that allows the ions in the electrolyte to polarize directly against the underlying conductive substrate. $\mathrm{Ni}(\mathrm{OH})_{2}$ only becomes conductive after oxidation to $\mathrm{NiOOH}$. 

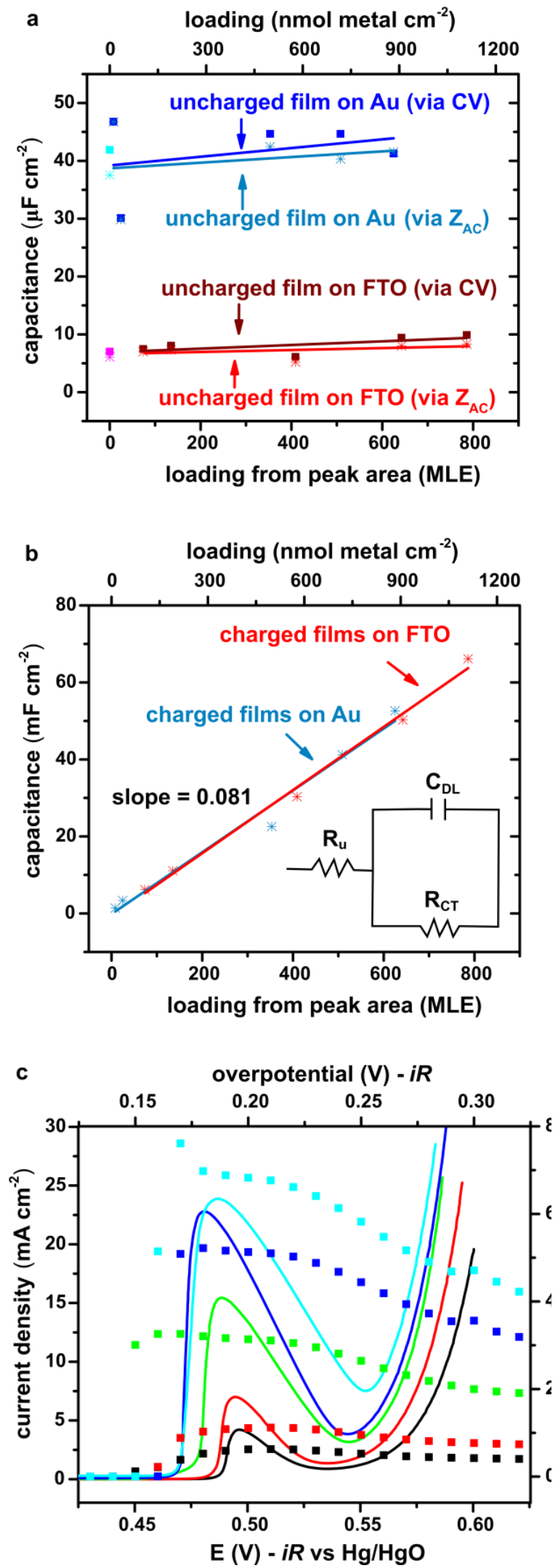

Figure 2.2. Capacitance of continuously deposited $\mathrm{Ni}(\mathrm{Fe})$ (oxy)hydroxide films as a function of loading for a) uncharged films deposited on $\mathrm{Au}$ (dark and light blue) and FTO (dark and light red) electrodes as determined via cyclic voltammetry $(\mathrm{CV}$, squares) and AC impedance spectroscopy $\left(Z_{\mathrm{AC}}\right.$, stars) with the data points at zero loading corresponding to the $C_{\mathrm{DL}}$ values of bare Au (aqua) and FTO (pink) electrodes and for $\mathrm{b}$ ) charged films deposited on $\mathrm{Au}$ (blue) and FTO (red) electrodes as determined via impedance spectroscopy. The inset displays the equivalent circuit used to fit the impedance data. c) Voltammetry, and the corresponding double layer capacitance values determined via impedance spectroscopy as a function of potential for the five $\mathrm{Au}$ films of varying loading shown in $a$ and $b$. 
To address this, we measured the film capacitances in the charged state at $0.52 \mathrm{~V}$ vs. $\mathrm{Hg} / \mathrm{HgO}$ where the $\mathrm{Ni}(\mathrm{Fe}) \mathrm{OOH}$ is electrically conducting using $\mathrm{AC}$ impedance spectroscopy (the voltammetry measurement of $C_{\mathrm{DL}}$ is not possible due to interference from redox and OER current in this range). Figure $2.2 \mathrm{~b}$ shows these $C_{\mathrm{DL}}$ are up to three orders of magnitude larger than those of the uncharged films, and increase linearly with loading. These data show that $C_{\mathrm{DL}}$ measurements are only a reliable indicator of ECSA when the electrocatalyst film has high electronic conductivity and that the benchmarking method reported for determining ECSA fails for hydrated electrolyte-permeable electrocatalysts if they are not conductive., ${ }^{5,36}$

We find from the slope of the fit lines in Figure 2.2b that the intrinsic areal capacitance of a single $\mathrm{Ni}(\mathrm{Fe}) \mathrm{OOH}$ nanosheet is $81 \mu \mathrm{F} \mathrm{cm}{ }^{-2}$ on both FTO and Au. This intrinsic capacitance calculated in this way includes contributions from both sides of the $\mathrm{Ni}(\mathrm{Fe}) \mathrm{OOH}$ nanosheet structure. Using a value of $81 \mu \mathrm{F} \mathrm{cm}{ }^{-2}$ to calculate loadings would result in a 1:1 correlation between the effective number of Ni monolayers determined by impedance spectroscopy and measurements on the same film using integration (under conditions described in detail in the next section) of the $\mathrm{Ni}$ redox wave assuming each $\mathrm{Ni}$ of the $\mathrm{Ni}(\mathrm{Fe}) \mathrm{OOH}$ structure is electrochemically active. This value of areal capacitance is within ranges reported in literature for metals in alkaline solutions, ${ }^{40,41}$ but is higher than the $40 \mu \mathrm{F} \mathrm{cm}^{-2}$ used by McCrory et al. ${ }^{5,36}$ and other reported values for Ni metal under alkaline conditions. ${ }^{42,43}$

The potential dependence of $C_{\mathrm{DL}}$ (Figure 2.2c) shows a dramatic increase at the $\mathrm{Ni}(\mathrm{Fe})(\mathrm{OH})_{2}$ oxidation potential and is correlated with the turn-on in electrical conductivity. ${ }^{7}$ The highest capacitance values are found near the Ni oxidation peak, and 
may contain a contribution from psuedocapacitance. ${ }^{40}$ The observation that the electrode capacitance does not track the oxidation wave observed in the voltammetry (and exhibits similar values during the reverse discharging process) indicates, however, that the capacitance is not solely due to $\mathrm{Ni}^{2+/ 3+}$ redox pseudocapacitance but is also associated with a more-classical non-faradaic charging of the conductive $\mathrm{Ni}(\mathrm{Fe}) \mathrm{OOH}$ nanosheets (in a similar way to that observed, e.g., for porous graphene electrodes). The reduced $C_{\mathrm{DL}}$ at higher potentials in the OER region (Figure 2.2c) may indicate the presence of oxygen bubbles which could reduce the surface area in contact with the electrolyte. We note also that the equivalent circuit used here was chosen for simplicity. More-complicated circuits treating the porous film as a resistor/capacitor network may be more appropriate if applied properly. ${ }^{40,44,45}$

Our conclusion from the capacitance studies is therefore that, for $\mathrm{Ni}(\mathrm{Fe}) \mathrm{OOH}$ systems that are electrolyte permeable and have potential-dependent conductivity, capacitance measurements can only be used to estimate ECSA or catalyst loading under potential regimes where the films are conductive. We note that related challenges in ECSA estimations by capacitance measurements have been discussed in the context of $\mathrm{Ni}(\mathrm{Fe}) \mathrm{O}_{x}$ nanoparticle electrodes previously. ${ }^{46}$ 


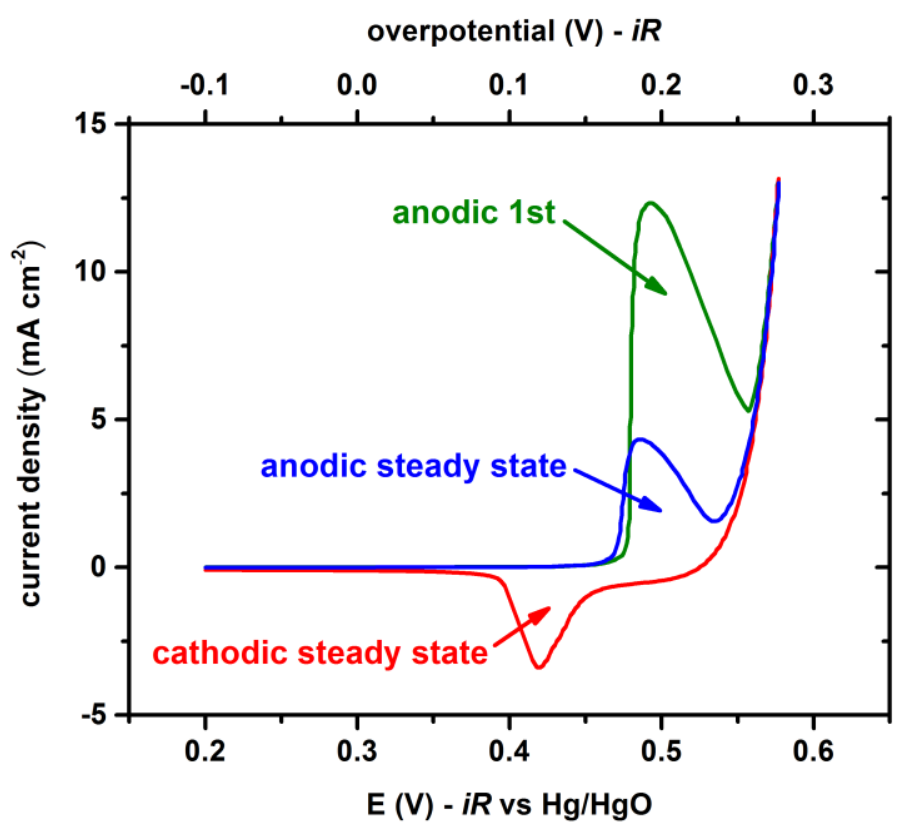

Figure 2.3. Voltammetry data for a continuously deposited $\mathrm{Ni}(\mathrm{Fe}) \mathrm{OOH}$ film with a thickness of $\sim 450$ MLE. The plot shows 1.5 cycles starting from a completely reduced film and then sweeping anodically. Any subsequent cycles would produce peaks that closely resemble those of the steady-state peaks.

Redox wave integration and charge reversibility.

The number of electrochemically active Ni sites can be estimated by integration of the Ni oxidation peak near $0.5 \mathrm{~V}$ vs. $\mathrm{Hg} / \mathrm{HgO} .{ }^{34}$ Figure 2.3 shows the representative voltammetry of a $\mathrm{Ni}(\mathrm{Fe}) \mathrm{OOH}$ film with a loading of 450 MLE. The integrated charge in the first anodic wave is larger than that in the second and all subsequent cycles. This change in peak size complicates analysis of the number of electrochemically active $\mathrm{Ni}$ species in the electrode film based on the integrated Ni redox wave. Following a cathodic deposition (driving nitrate reduction to locally raise the $\mathrm{pH}$ and precipitate hydroxide), all of the $\mathrm{Ni}$ in the film is expected to be in the $2^{+}$oxidation state according to the $\mathrm{Ni}$ Pourbaix diagram. ${ }^{47}$ As the electrode is swept anodic the Ni is oxidized, with the onset determined by the identity and concentration of metal dopants, as well as the mass 
loading. ${ }^{7,21,34}$ As the $\mathrm{Ni}$ is oxidized to the nominally $3^{+}$or $4^{+}$oxidation state, it becomes electronically conductive and the entire film is able to participate in the OER at sufficiently positive potentials. When the cell potential is swept cathodically, however, the film becomes insulating as the Ni returns to the $2^{+}$state, and a portion of the film is trapped irreversibly (on the timescale of the voltammetry scan) in the oxidized state by the insulating $\mathrm{Ni}(\mathrm{OH})_{2}$ layer as depicted in Figure 2.4. ${ }^{7}$ When the potential is swept anodically on subsequent scans, only the Ni ions that have been reduced (plus possibly a small number of ions reduced via self-discharge) can be re-oxidized. This behavior has been discussed by Corrigan et al. and Barnard et al. ${ }^{34,48,49}$ Since the number of Ni ions able to be oxidized is now determined by the number reduced in the preceding cathodic sweep, and not by the number total number of Ni ions in the film, all subsequent anodic peaks display the same steady-state behavior. Thus any estimations of loading based on the steady-state integrated peak area would underestimate the number of $\mathrm{Ni}$ atoms in the film when the reversibility is less than unity. 


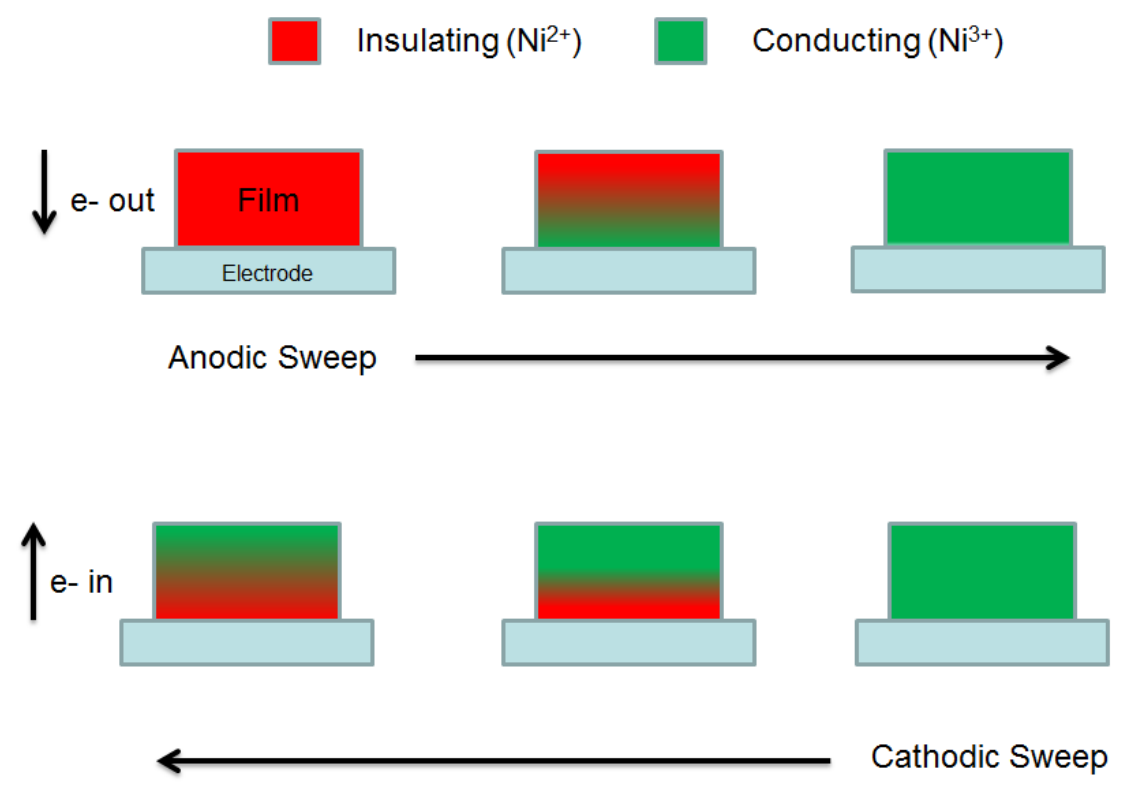

Figure 2.4. Schematic representation of how changes in film conductivity could affect the magnitude of the steady-state $\mathrm{Ni}^{2+/ 3+}$ redox waves. During the oxidation sweep, the film becomes conductive as an oxyhydroxide and the entire film is oxidized. During the return reductive sweep, the film becomes electrical insulating as a hydroxide. Because the regions of the film nearest to the electrode surface are reduced first, this leaves regions of the films trapped in the oxidized state for the thicker films.

Concurrent QCM measurements were used to confirm that the changing redox wave integration cannot be explained by mass loss in the film and that the (lower limit) average number of electrons transferred per $\mathrm{Ni}$ in the first oxidation wave is $\sim 1$ (accounting for the Fe content of the film, but ignoring additional mass associated with the absorbed water or ions). ${ }^{50}$ In other work the presence of quadravalent nickel, and an average of 1.67 electrons being transferred has been indicated, ${ }^{48}$ consistent with our lower-limit QCM measurement. The results displayed in Figure 2.5 indicate that the number of metals determined from the first anodic peak match well with the number of metals as determined by QCM, as long as sufficiently slow scan rates $\leq 10 \mathrm{mV} \mathrm{s}^{-1}$ are used. This result demonstrates that the Ni atoms are uniformly electrochemically accessible within the catalyst film. Because the Fe content in the film is no higher than 
$\sim 15 \%$, less than the solubility limit in $\mathrm{NiOOH}$ estimated at $>20 \%$ by Friebel et al, ${ }^{25}$ the electrochemical accessibility of the Ni cations implies similar electrochemical accessibility of the Fe cations throughout the film for all thicknesses studied here. This conclusion is also consistent with the capacitance data as a function of loading in Figure 2.2b. Because of the excellent correlation between the number of Ni determined by integration of the first $\mathrm{Ni}$ oxidation wave (assuming $\sim 1 \mathrm{e}$ - per $\mathrm{Ni}$ ) and from the mass measurement, in all subsequent analyses we determine the Ni loading from the integrated charge in the first anodic redox wave.

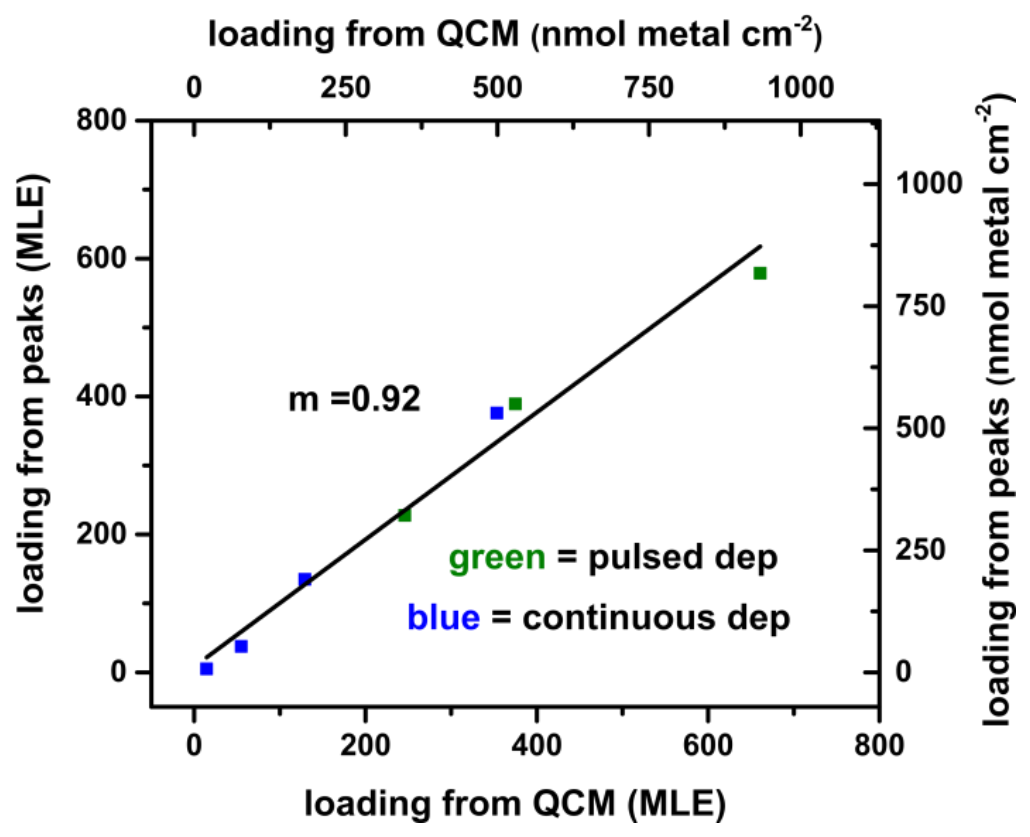

Figure 2.5. Comparison of loadings as determined by QCM and redox peak integration for both pulse (green) and continuously (blue) deposited films assuming a $1 \mathrm{e}^{-}$per $\mathrm{Ni}$ oxidation and the appropriate amount of Fe (see experimental). The loading on both axes is given in monolayer equivalents (i.e. MLE) - one MLE is the number of metal ions needed to form a single $\mathrm{MOOH}$ monolayer on the electrode.

We found that the electrochemical reversibility of the Ni oxidation wave was strongly affected by the film thickness. We define the electrochemical reversibility as: 


$$
\chi_{\mathrm{rev}}=\frac{C_{1}}{A_{1}}
$$

$A_{1}$ is the charge passed in the oxidation wave measured during the first anodic sweep of the film, and $C_{1}$ is the charge passed in the reduction wave upon the first return cathodic sweep. Reversibility expressed in this way is equivalent to the more-frequently reported coulombic efficiency or charge ratio. As discussed earlier, following the first oxidation wave, steady-state cycling is reached, and the all subsequent cathodic and anodic peak integrations yield very similar charge. Corrigan also reported reversibility for different metals co-deposited with $\mathrm{Ni}(\mathrm{OH})_{2}{ }^{34}$ Fully reversible films have $\chi_{\text {rev }}=1 . \chi_{\text {rev }}<1$ indicates that a portion of the film is trapped in the oxidized state, which can be directly visually observed in thicker films due to the dark coloration of $\mathrm{NiOOH}$ relative to $\mathrm{Ni}(\mathrm{OH})_{2} \cdot{ }^{51}$ 


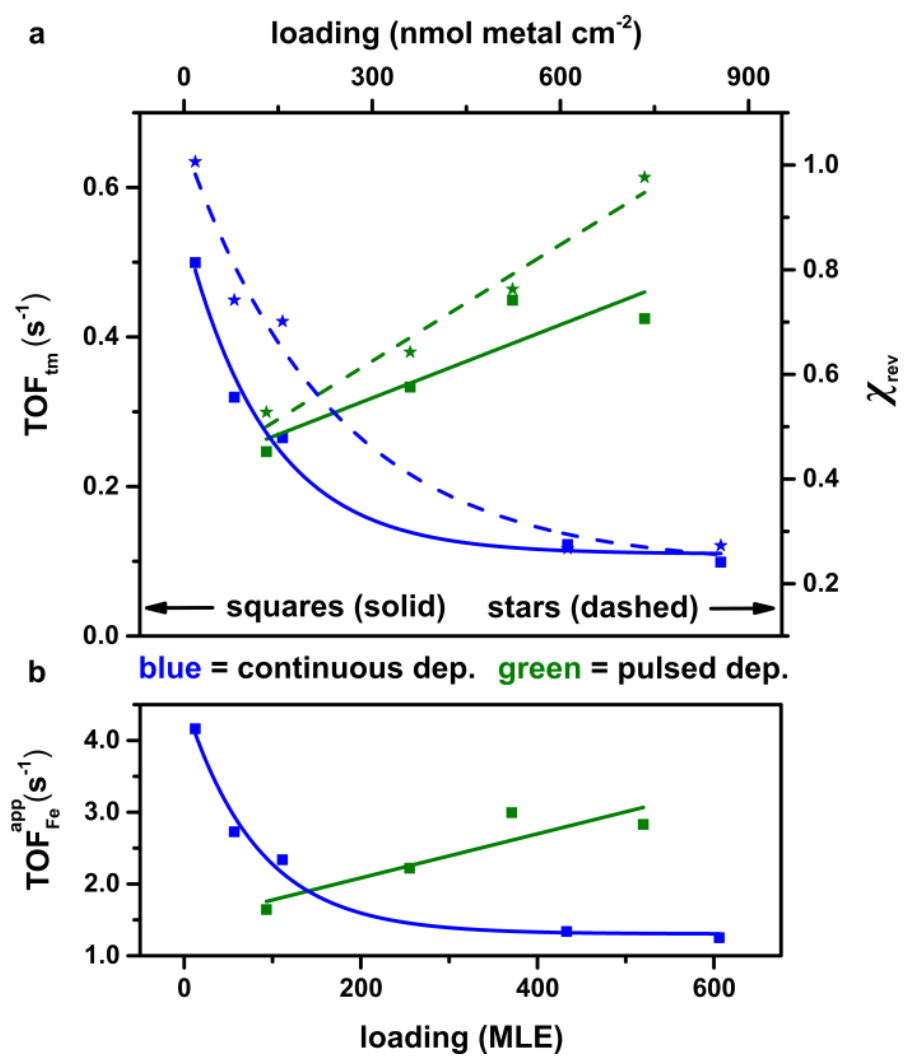

Figure 2.6. a) Loading-dependent reversibility (stars, dashed lines) and activity (squares, solid lines) of pulse (green) and continuously (blue) deposited films. $\chi_{\text {rev }}$ is defined in Eqn. 4. The data shown was collected on Au electrodes at $10 \mathrm{mV} \mathrm{s}^{-1}$. The activities were calculated from chronoamperometry data ( $i R_{\mathrm{u}}$ corrected) at $300 \mathrm{mV}$ overpotential and normalized with a) total metal loadings determined using integration of the anodic wave in the first cycle, and b) total Fe loadings derived from Fe concentrations obtained through XPS. The lines are drawn to illustrate trends.

Figure 2.6a shows $\chi_{\text {rev }}$ for $\mathrm{Ni}(\mathrm{Fe}) \mathrm{OOH}$ films as a function of loading (dashed lines). For continuously deposited films with small loading $\chi_{\mathrm{rev}}$ is $\sim 1$, while as the thickness increases $\chi_{\text {rev }}$ decreases. The observation of $\chi_{\text {rev }}<<1$ at high loading provides insight into loss mechanisms operating within the electrode film. For example, if the ionic resistivity in the film $R_{\text {ion }}$ were much larger than the film's electrical resistivity $R_{\text {film, }}$ the electrochemical reduction of the film from electrically conducting $\mathrm{Ni}(\mathrm{Fe}) \mathrm{OOH}$ to insulating $\mathrm{Ni}(\mathrm{Fe})(\mathrm{OH})_{2}$ would start from the film/solution interface and proceed inward until the last $\mathrm{Ni}$ or $\mathrm{Fe}$ ions near the electrode/film interface were reduced, resulting in $\chi_{\text {rev }}$ 
$=1$. In the opposite case, with $R_{\text {film }}>R_{\text {ion }}$, the film would be reduced from the electrode/film interface outward. In this scenario, with the buildup of an insulating layer near the electrode surface preventing the reduction of $\mathrm{Ni}$ ions near the film/solution interface, $\chi_{\text {rev }}$ would be $<1$, consistent with what is reported here. This suggests that the electrical connectivity of the film is a greater contributor to $\eta_{\text {total }}$ than mass transport of ions into and out of the film in $1 \mathrm{M} \mathrm{KOH}$ for the continuously deposited films.

For the pulse-deposited films, $\chi_{\text {rev }}$ increases with loading, opposite to the behavior observed for the continuously deposited films. As discussed below, this may be due to better electrical interconnection of the denser-packed films resulting from pulsed deposition.

We further note that in the thin-film limit when $\chi_{\text {rev }}$ approaches unity any of the $\mathrm{Ni}$ redox waves can be used to measure the number of electrochemically active $\mathrm{Ni}$ species in the films. In the thick-film limit were $\chi_{\text {rev }}<1$, it is essential to estimate loading from the first anodic sweep after fully reducing the film, which we accomplish by holding at $-1.0 \mathrm{~V}$ vs. $\mathrm{Hg} / \mathrm{HgO}$ for $\sim 20 \mathrm{~min}$. For the films studied here, with nominally $10-15 \% \mathrm{Fe}$ in $\mathrm{NiOOH}$, the Ni redox peak is only minimally obscured by the onset of OER current (Figure 2.3). For higher Fe content films ${ }^{34}$ the Ni oxidation wave can be significantly obscured by the onset of OER, making peak integration difficult. For thin films the loading can still be assessed through integration of the cathodic wave. Alternatively, QCM measurements can be used in these cases. 


\section{Intrinsic Activity of Catalyst Films.}

Understanding how film performance varies with loading on a per-metal basis is important because two key applications of OER catalysts, photoelectrochemical cells (PECs) and electrolyzers, operate at the extremes of catalyst loading. For PECs, the catalyst parasitically absorbs light, which forces loading to a minimum. ${ }^{51}$ For electrolyzers, high current densities require the use of large loading to minimize overpotential..$^{52}$

Figure 2.6 shows the loading-dependent $\mathrm{TOF}_{\mathrm{tm}}$ and $\mathrm{TOF}_{\mathrm{Fe}}^{\mathrm{app}}$ data from pulsed and continuously deposited films, along with the charge reversibility from the same films discussed above. The pulse-deposited films increase in both TOF metrics with loading while the continuously deposited films TOF values decrease. The TOF data, interestingly, showed the same trend observed in the reversibility as a function of thickness.

While in principle both $\mathrm{TOF}_{\mathrm{tm}}$ and $\mathrm{TOF}_{\mathrm{Fe}}^{\mathrm{app}}$ should be an intrinsic property of a catalyst, the observation that the measured values depend on loading indicates heterogeneity in the microenvironments of the catalyst active sites in the film that depend on the deposition method. The decrease in TOF with thickness for the continuously deposited films might be explained by increased electrical resistance through the thicker films lowering the available overpotential to drive the OER reaction for $\mathrm{Ni}(\mathrm{Fe}) \mathrm{OOH}$ active sites further from the electrode surface. The increase in TOF for the pulsedeposited films, however, suggests an increase in the intrinsic activity with thickness, which could be caused by changes in the nanostructure/morphology as the pulsed deposition proceeds. The local composition also depends on the deposition time and hence film thickness, as we discuss below. 
Physical Characterization of Pulse and Continuously Deposited $\mathrm{Ni}(\mathrm{Fe})(\mathrm{OH})_{2}$.

To examine the structure of the films, both top-down and cross-sectional scanning electron microscopy (SEM) images were collected (Figure 2.7). The images show significant differences in the micro and nanoscale morphology of the films, with nanoscale platelets characteristic of electrodeposited hydroxides with a brucite-like structure apparent in both.
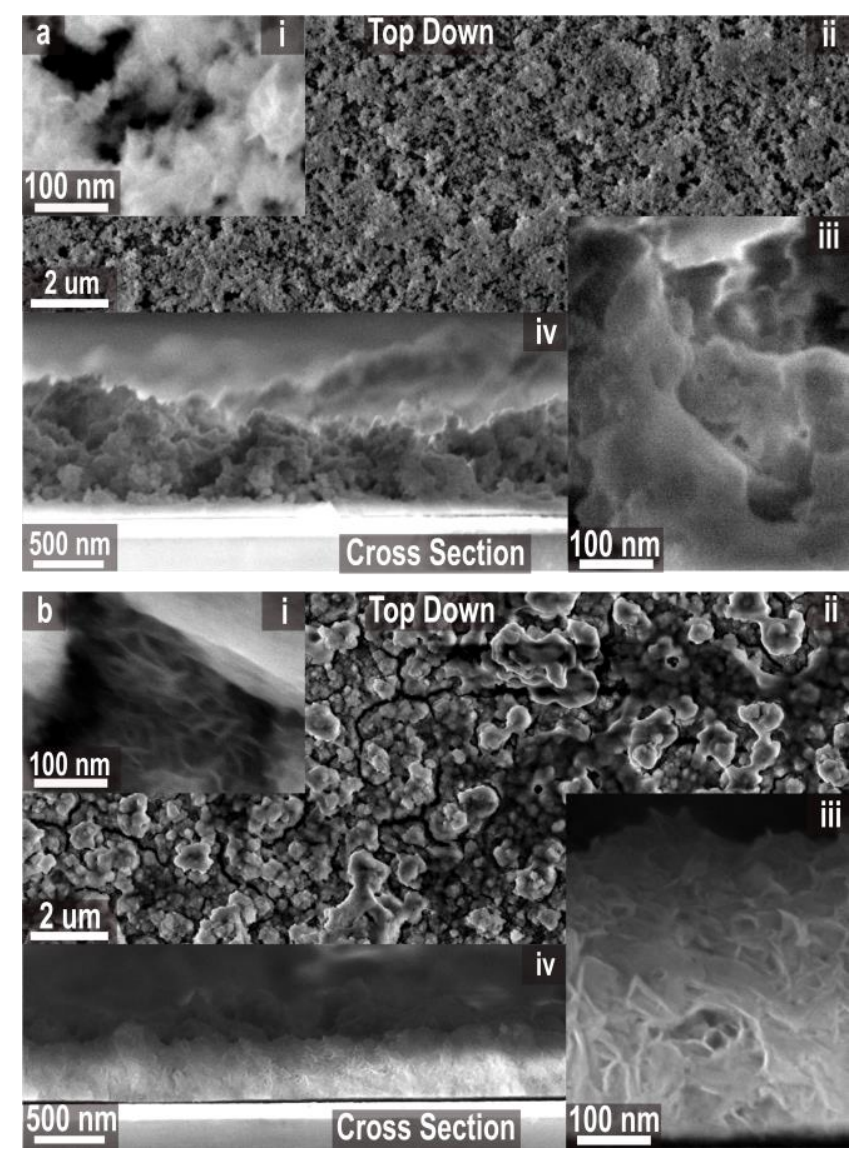

Figure 2.7. SEM Images of thick a) continuously and b) pulse-deposited films. Both topdown (i-ii) and cross-sectional (iii-iv) images are shown. The films were deposited at conditions that resulted in effective thicknesses of $\sim 600$ MLE (continuous) and $\sim 520$ MLE (pulsed). 
Figures $2.7 \mathrm{a}$, i and $7 \mathrm{a}$,ii show top-down views of the continuously deposited film that indicate a broccoli-like morphology where small bundles of $\mathrm{Ni}(\mathrm{Fe}) \mathrm{OOH}$ are deposited on top of one another. The inefficient packing that results can be seen in the cross-sectional images (Figures 2.7a,iii and 2.7a,iv) that show the presence of large vertical and horizontal voids.

The pulse-deposited films are composed of larger nodules (Figure 2.7b,ii) that also appear denser in the magnified top-down image (Figure 2.7b,i). The cross-sectional images in Figure 2.7b,iii-iv show that these features are highly interconnected with much fewer voids than the continuously deposited films.

The opposite trends in both TOF metrics and reversibility with loading shown in Figure 2.6 might be explained by the morphology differences. For continuous deposition, the low catalyst packing density and large void volume can be explained by the deposition mechanism whereby nitrate reduction driven at the conductive electrode surface leads to a local increase in $\mathrm{pH}$ and precipitation of metal hydroxide. With time that $\mathrm{pH}$ gradient diffuses further into the bulk electrolyte causing continued precipitation of hydroxides on the outer surface of the film. The solution near the electrode surface is depleted of $\mathrm{Ni}$ and $\mathrm{Fe}$ nitrate and thus voids are formed in the growing film. This might lead to poor electrical connectivity from the outermost portions of the film to the conductive electrode, resulting in increased film electrical resistance and lower TOF and charge reversibility.

The pulse-deposited films are different due to both the vertical and horizontal connectivity provided by the more-densely packed structure. This is likely because fresh metal nitrate solution infiltrates the previously deposited material during the stirring steps 
between deposition current pulses. The denser films remain electrolyte-porous with all metal cations electrolyte accessible, but might provide better electrical connectivity that leads to the higher reversibility and higher TOF for thick films.

It is interesting to note that our recent in-situ measurements of the average electrical conductivity of $\mathrm{Ni}(\mathrm{Fe}) \mathrm{OOH}$ under OER conditions ${ }^{7}$ indicate that while passing $50 \mathrm{~mA} \mathrm{~cm}^{-2}$, less than a $0.5 \mathrm{mV}$ drop over the thickest films studied here is expected based on Tafel slopes of between 33 and $41 \mathrm{mV} \mathrm{dec}^{-1}$ for $\mathrm{Ni}(\mathrm{Fe}) \mathrm{OOH}$. This is not sufficient to cause a factor of five decrease in $\mathrm{TOF}_{\mathrm{tm}}$ as observed for the continuously deposited films with large loading. However, this does not rule out the possibility of higher local resistivity, particularly in the outer portions of the films. To account for the entire decrease in performance exhibited between the films with the highest and lowest loadings would require a $\sim 25$-fold decrease in the average conductivity of the film from that measured earlier.

Another possible contributor to the different loading-dependent behavior is a variable $\mathrm{Fe}$ content within the film. If Fe preferentially deposits compared to $\mathrm{Ni}$, then the innermost layers of the film may have more Fe sites than the outermost layers. This would be the result of local depletion of the $\mathrm{Fe}^{2+}$ in the deposition solution in the absence of stirring. Since Fe incorporation is essential for the high activity of Ni-based films, ${ }^{7,25,28,53-55}$ this could have a significant impact on the loading-dependent behavior.

The Fe content of thin ( $\sim 50$ MLE) and thick ( 500 MLE) films deposited using both continuous and pulsed deposition was thus investigated using XPS. For pulsedeposited films, the Fe content is $\sim 15 \%$ for both the low and high loading films. The continuously deposited films, however, show $12 \%$ and $4 \%$ Fe in low and high loading 
films, respectively. As the penetration depth of XPS is only a few nanometers, ${ }^{56}$ and the highest loading films approach a micron in thickness, the average Fe content in the continuously deposited high-loading film lies somewhere in-between the 12 and $4 \%$. This data therefore shows that a way to counteract compositional inhomogeneity when depositing $\mathrm{Ni}(\mathrm{Fe}) \mathrm{OOH}$ from mixed-metal solutions is to use short electrochemical pulses allowing sufficient time (aided by stirring) in between depositions to replenish the local ion concentrations.

Recent work showing the variation in current density with Fe content in Ni-based films ${ }^{54}$ allows us to approximate the effect on $\mathrm{TOF}_{\mathrm{tm}}$ from the drop in Fe content independent of other variables. By assuming an average Fe content of $7 \%$ in the thickest continuously deposited films, the decrease in current density predicted would account for only around half of the observed decrease in $\mathrm{TOF}_{\mathrm{tm}}$. Previous work from our group also shows a similar decrease in $\mathrm{TOF}_{\mathrm{tm}}$ with increased loading for $\mathrm{Fe}$-free $\mathrm{Ni}$ films ${ }^{7}$, again suggesting that the local Fe concentration doesn't fully explain the observed trends. $\mathrm{TOF}_{\mathrm{Fe}}^{\mathrm{app}}$ also decreases with loading for the continuously deposited films and increases for the pulse-deposited films (Figure 2.6b).

Another possible effect is the enhancement of activity by synergistic filmsubstrate effects as discussed by Bell and coworkers. ${ }^{31}$ However, given that the effective length of this interaction was limited to the first 40 MLEs in Ni-based films, ${ }^{31}$ that the effect is reduced substantially for Fe-doped films, ${ }^{7}$ and that we find similar thickness dependence on both FTO and Au substrates here for the continuously deposited films (with no decrease in TOF with loading for pulse-deposited films), we do not believe substrate effects to be important in affecting the observed TOF data here. 


\section{Conclusion and Bridge}

We showed that $\mathrm{Ni}(\mathrm{Fe}) \mathrm{OOH}$ films deposited using a simple electrochemical pulsed-deposition technique enabled high practical performance comparable to $\mathrm{Ni}(\mathrm{Fe}) \mathrm{OOH}$ films deposited onto more complicated OER inert supports. To understand the intrinsic activity of the catalysts on a per-metal basis, several methods for determining electrochemically active surface areas were evaluated. Measurements based on the film capacitance, as well as those based on integration of the Ni redox wave, are complicated substantially by the fact that the catalyst is electrically insulating in the reduced state but conductive while oxidized and driving the OER. We found that capacitance measurements must be made when the catalyst is oxidized and conductive, while those based on redox-wave integration must be made after the film has been fully discharged through extended cathodic polarization to ensure that no fraction of the film is trapped in the oxidized state. Using these methods to measure loading, we discovered that the TOF (both on a total-metal or Fe-only basis) for pulse-deposited films increased with increasing loading, whereas continuously deposited films displayed the opposite behavior. The increase in TOF with loading for pulse-deposited films confirms previous work showing that substrate-activity-enhancement for high-loading $\mathrm{Ni}(\mathrm{Fe}) \mathrm{OOH}$ films is minimal. ${ }^{7}$ By analyzing the morphology and composition of the two types of films, the different loading-dependent trends in activity were explained by differences in (1) Fe incorporation (with the pulsed deposition yielding more-uniform Fe incorporation) and (2) morphology, with pulsed deposition providing denser films that are better electrically interconnected. We also discovered that the charge reversibility, a measure of the OER 
catalyst's ability to efficiently transfer ions and electrons through the film, showed loading-dependent behavior that correlated well with the TOF for the OER. Reversibility could therefore be a valuable metric for assessing different catalyst structures for efficient use of high mass loading. The findings here will thus help enable the further design of advanced electrodes with very high loading of $\mathrm{Ni}(\mathrm{Fe}) \mathrm{OOH}$ while maintaining high intrinsic activity.

Having investigated the deposition dependent behavior of $\mathrm{Ni}(\mathrm{Fe})$-based catalyst films, we have seen the bulk morphology of the films is vastly different, and likely a source of the different loading dependent activities. However, we are left with unanswered questions as to whether the local structure is similar under the two different deposition techniques employed. Chapter III will look make use of high-energy x-ray scattering to obtain information about the local environments in these films as a function of deposition technique, Fe content, and the number of electrochemical pulses. 


\section{CHAPTER III}

\section{DOMAIN STRUCTURES OF NI AND NIFE (OXY)HYDROXIDE OXYGEN- EVOLUTION CATALYSTS FROM X-RAY PAIR DISTRIBUTION FUNCTION ANALYSIS}

Portions of this chapter were previously submitted as Batchellor, A.S.; Kwon, G.; Tiede, D. M.; Boettcher, S. W. Domain Structures of Ni and NiFe (Oxy)Hydroxide Oxygen-Evolution Catalysts from X-ray Pair Distribution Function Analysis. J. Phys. Chem. C. 2016, Submitted. A.S.B. processed and analyzed all of the raw data and wrote the manuscript. S.W.B. was the principal investigator and provided editorial assistance. D.M.T. and G.K collected the raw data and provided training on PDF analysis techniques.

\section{Introduction}

Electrochemistry provides a mechanism to store energy generated by intermittent renewable sources such as solar or wind..$^{1-3}$ Water splitting to form hydrogen fuel, via either a photoelectrochemical device or an electrolyzer, is attractive because it allows for a carbon-free renewable energy storage cycle. One challenge in water splitting is minimizing the overpotential associated with the oxygen-evolution reaction (OER). ${ }^{4-6}$ Inexpensive, earth-abundant transition-metal-based catalysts are being explored which 
outperform their precious-metal counterparts in alkaline media, ${ }^{7}$ suggesting routes to large-scale advanced alkaline electrolysis technologies. ${ }^{8-12}$

A large number of Ni-based OER catalysts have been reported including oxides, ${ }^{7}$ (oxy)hydroxides, ${ }^{13}$ phosphides, ${ }^{14}$ selenides, ${ }^{15}$ and sulfides. ${ }^{16}$ Their synthesis is accomplished through electrodeposition, ${ }^{17-20}$ sol-gel, ${ }^{21}$ solvo- and hydrothermal, ${ }^{22-26}$ or photochemical techniques. ${ }^{27}$ Regardless of the starting material or reaction conditions employed, similar thermodynamically stable phases, i.e. (oxy)hydroxides in alkaline media, ${ }^{28}$ are likely to form, at least at the surface. Many oxides, for example, typically reach this (oxy)hydroxide through the formation of a disordered hydrous layer which gradually grows into the material from the film/electrolyte interface. ${ }^{29-31}$

Fe impurities are also ubiquitous in alkaline electrolyte, except in cases where extreme care is taken to remove them. ${ }^{13} \mathrm{Fe}$ cations incorporate within the $\mathrm{Ni}$ and Co (oxy)hydroxide phases to dramatically enhance activity. ${ }^{17,32}$ Such (oxy)hydroxides can also be synthesized directly, and the resulting (oxy)hydroxides appear to be porous and electrolyte-permeated with interconnected locally ordered domains (e.g. nanosheet fragments) interspersed with electrolyte. Using in situ conductivity measurements we showed that while $\mathrm{Ni}$ and Co oxyhydroxides are electrically conductive at low OER overpotentials, pure FeOOH is not. ${ }^{17}$ We therefore proposed a working model where the $\mathrm{NiOOH}$ serves as an electrically conductive and electrolyte-porous host for Fe enabling synergistic Ni-Fe interactions for the (nominally) Fe active sites, ${ }^{33}$ in accord with related work on these systems. ${ }^{34-37}$ A detailed understanding of the intermediate and extended structural units in $\mathrm{Ni}(\mathrm{Fe}) \mathrm{OOH}$ and related mixed-metal (oxy)hydroxides is, however, 
missing. Such understanding is important for developing the synthetic design rules to improved catalysts. ${ }^{38,39}$

The experimentally measured overpotential for the OER is affected by the intrinsic active-site kinetics, as well as mass and electron transport to the active catalyst sites. Minimization of the kinetic overpotential relies on tuning the active site chemistry/energetics and increasing their density. Local structural modifications (i.e., changes in the nearest-neighbor atom distances) due to substitution of metals in Ni-based systems were observed through EXAFS, ${ }^{34,40}$ and significant effort has been devoted to optimizing the chemical composition of OER catalysts, with mono- through quaternary metal oxide/(oxy)hydroxide systems having been investigated. ${ }^{17,41,42}$ Domain-size effects are also likely important in determining active-site density, as computational studies have predicted open edge sites to be active sites for $\mathrm{Ni}(\mathrm{Fe}) \mathrm{OOH}$ and related nanosheets. ${ }^{34,43}$

Mass and electron transport to the active sites is determined by the catalyst's intermediate structure and morphology/architecture. Differences in mesoscale features, for example, are evident from scanning electron microscopy. ${ }^{44,45}$ The study of intermediate length scales with traditional x-ray diffraction techniques has been difficult, with measurements showing only a small number of broad peaks. The phases that form are thus often referred to as amorphous. ${ }^{24,46,47}$

With synchrotron x-ray sources, total-scattering experiments coupled with pair distribution function (PDF) analysis allow the study of disordered OER catalysts ${ }^{48}$ and provide architectural details that are not available from traditional crystallographic analysis. For example, Co (oxy)hydroxide OER catalysts have been recently studied using PDF analysis, with resolved structures demonstrating anion-dependent size and 
stacking behavior, ${ }^{49}$ leading to loading-dependent performance, ${ }^{50}$ with the presence of distorted edge sites proposed as a source of high activity. ${ }^{51}$ Neilson studied nominally “amorphous" $\mathrm{FeO}_{x}$ OER catalysts synthesized from photodecomposition of organometallic precursors and used PDF analysis to show the structure contains both amorphous and defective-spinel-lattice features. ${ }^{52}$ Ir and Mn oxide water oxidation catalyst have also been studied. ${ }^{53,54}$

Here we report the first PDF analysis on $\mathrm{Ni}_{(1-z)} \mathrm{Fe}_{z} \mathrm{O}_{x} \mathrm{H}_{y}$ electrocatalysts, the highest performing OER films in basic media, as a function of electrochemical deposition method, cycling and Fe content. The PDF patterns presented (1) are well fit by simple modifications to a (brucite) $\mathrm{Ni}(\mathrm{OH})_{2}$ unit cell, (2) show a degree of intersheet correlation that depends on the presence of Fe cations, (3) demonstrate the ability to cycle between as-deposited, oxidized and reduced states with no apparent loss in domain size or structure, and (4) show a dependence of domain size on the number of electrochemical pulses applied during deposition. These results thus support a view of a catalyst structure that consists of two-dimensional $\mathrm{Ni}(\mathrm{Fe}) \mathrm{OOH}$ nanosheet fragments, which are electrically interconnected in a porous electrolyte-permeated catalyst network. The work also suggests, due to the relative similarity of the PDF patterns obtained under different conditions, that the differences in activity observed for different electrodeposition protocols $^{55}$ are due primarily to how the catalyst sheets are interconnected and not due to dramatic differences in domain structure. 


\section{Experimental}

\section{Electrode Fabrication.}

Conductive Au-coated electrode substrates were prepared by first cleaning glass slides in a 3:1 hydrogen peroxide to conc. sulfuric acid (piranha) solution followed by plasma etching. Next, $10 \mathrm{~nm}$ of Ti and $100 \mathrm{~nm}$ of Au were deposited by electron-beam evaporation. The Au/Ti coated glass slides were then cut into $\sim 8 \mathrm{~cm}^{2}$ rectangles. $\mathrm{Cu}$ wire was attached to the slides via Ag paint. Exposed wire and Ag paint were sealed with epoxy.

\section{Film Deposition.}

The films were cathodically deposited from a $0.1 \mathrm{M}$ solution (total metals basis) prepared by mixing $\mathrm{Ni}\left(\mathrm{NO}_{3}\right)_{2} \cdot 6 \mathrm{H}_{2} \mathrm{O}$ (Alfa Aesar) and $\mathrm{FeCl}_{2} \cdot 4 \mathrm{H}_{2} \mathrm{O}$ (Sigma-Aldrich) in a 100:0, 95:5, 90:10 or 85:15 (mol Ni : mol Fe) ratio in 18.2 $\mathrm{M} \Omega \mathrm{cm}$ water. Continuous depositions from the 5, 10, and $15 \%$ Fe solutions resulted in 8,16 , and $23 \%$ Fe in the films. Pulsed-deposited films from 5\% Fe solution resulted in $15 \% \mathrm{Fe}$ in the films. The deposition solution was sparged with $\mathrm{N}_{2}$ for 20 min prior to film deposition. All films were deposited at a cathodic current density of $-25 \mathrm{~mA} \mathrm{~cm}^{-2}$. Continuously deposited films were subjected to reducing potentials for $10 \mathrm{~s}$. Pulse-deposited films were subjected to sequential $2 \mathrm{~s}$ cathodic current pulses at $-25 \mathrm{~mA} \mathrm{~cm}^{-2}$. Following each pulse, the solution was stirred for $10 \mathrm{~s}$ to ensure homogeneity of the deposition solution prior to the next pulse. All pulse-deposited films were synthesized using five cycles unless otherwise 
noted. All films were briefly dipped into ultrapure water to remove residual metal ions prior to electrochemical testing.

\section{Film Conditioning.}

$\mathrm{Ni}_{(1-z)} \mathrm{Fe}_{z} \mathrm{O}_{x} \mathrm{H}_{y}$ films were examined under three different states: as deposited, oxidized, and reduced. As deposited films were quickly dipped in ultrapure water following deposition, and no electrochemical conditioning was performed. Oxidized films were subjected to a $1 \mathrm{mV} / \mathrm{s}$ linear sweep from 0.35 to $0.65 \mathrm{~V}$ vs. $\mathrm{Hg} / \mathrm{HgO}$, and then held at $0.65 \mathrm{~V}$ for $30 \mathrm{~s}$ to ensure complete oxidation. Reduced films were oxidized according to the procedure described above, and were then subjected to a $20 \mathrm{mV} / \mathrm{s}$ linear sweep from $0.65 \mathrm{~V}$ to $-0.5 \mathrm{~V}$ vs. $\mathrm{Hg} / \mathrm{HgO}$, and then held at $-0.5 \mathrm{~V}$ until the reduction current stabilized at a constant value, and the transparent color of the pure $\mathrm{Ni}^{2+}$ film was observed over the entire sample (the oxidized films are dark brown/black in color). Both the oxidized and reduced films were dipped in ultrapure water following conditioning and then dried with a $\mathrm{N}_{2}$ stream. The $\mathrm{Ni}$ and $\mathrm{NiFe}$-based oxidized films maintained their dark color throughout the PDF measurement, indicating a predominantly $3^{+} / 4^{+} \mathrm{Ni}$ oxidation state, compared to the light-green reduced $\mathrm{Ni}$ and $\mathrm{NiFe}-$ based films.

\section{Ex-Situ X-Ray Sample Preparation.}

The dried films were scrapped off the electrode using a razor blade, and then transferred to quartz capillary tubes with diameters of 1.0 or $0.7 \mathrm{~mm}$ (Charles Supper). 1 $\mathrm{M} \mathrm{KOH}$ was added to the dried film (to match the alkaline OER environment) and the 
contents of the tube were centrifuged until the material was tightly packed and the $\mathrm{KOH}$ had been dispersed throughout the catalyst powder.

High Energy X-ray Scattering Measurements and Pair Distribution Function Analysis.

High energy X-ray (58 keV, $\lambda=0.2128 \AA$ ) scattering measurements were performed at beamline 11-ID-B of the Advanced Photon Source (APS). Scattering patterns were measured as a function of the scattered wave vector $q$, where $q=4 \pi$ $\sin (\theta) / \lambda$, and $2 \theta$ is the scattered angle. The sample scattering patterns were corrected for solvent, container, and instrument background scattering, and then for multiple scattering, X-ray polarization, sample absorption, and Compton scattering using the programs PDFgetX $2^{56}$ and PDFgetX $3,{ }^{57}$ with $q_{\min }$ and $q_{\max }$ set to $0.8 \AA^{-1}$ and $21 \AA^{-1}$ respectively.

Model PDF Calculations. Models for the $\mathrm{Ni}_{(1-z)} \mathrm{Fe}_{z} \mathrm{O}_{x} \mathrm{H}_{y}$ films were created by modifying a brucite $\mathrm{Ni}(\mathrm{OH})_{2}$ structure (ICSD entry 109391). Crystal Maker software was used to convert the $\mathrm{Ni}(\mathrm{OH})_{2}$ extended structure to a molecular unit of defined size, which was saved in protein data bank (.pdb) format. Scattering patterns for the model structures were created using SolX. ${ }^{58,59}$ A residual function, or goodness of fit, $R_{\mathrm{w}}$, was calculated using

$$
R_{\mathrm{w}}=\sqrt{\frac{\Sigma\left(G(r)_{\text {model }}-G(r)_{\mathrm{exp}}\right)^{2}}{\Sigma\left(G(r)_{\exp }\right)^{2}}}
$$

to quantify the degree to which the simulated model PDF patterns fit the experimental data, where $G(r)_{\text {model }}$ and $G(r)_{\exp }$ are the generated and experimental PDF patterns, respectively. 


\section{Results and Discussion}

Modeling of $\mathrm{Ni}_{(1-z)} \mathrm{Fe}_{z} \mathrm{O}_{x} \mathrm{H}_{y}$ Structures.

Elucidating the intermediate structure of OER catalysts is important for understanding the effects of mass and electronic transport, as well as defect and edge sites, in catalyst performance. The different Ni (oxy)hydroxide structures are well represented by the so-called Bode diagram, ${ }^{60}$ with variations in intersheet spacing, Ni-O bond length, and the presence of turbostratic disorder differentiating the four phases. Since Ni and Fe have similar atomic numbers and atomic scattering factors all models were constructed with $\mathrm{Ni}$ as the only cation, and the scattering from protons was ignored. To capture the range of structural features observed we started with $\beta-\mathrm{Ni}(\mathrm{OH})_{2}$ (ICSD 109391) and then adjusted the unit cell's (Figure 1b) $a$-parameter to fit the Ni-Ni bond distance (second peak in the PDF pattern), the $z$-fractional coordinate of the $\mathrm{O}$ atoms inside the unit cell to fit the Ni-O bond length (first peak in the PDF pattern), and the $c$ parameter to account for the intersheet spacing and degree of structural coherence between the sheets.

The molecular sheet structures used to simulate PDF patterns were constructed by altering the $\mathrm{Ni}(\mathrm{OH})_{2}$ unit cell such that the $<001>$ planes of $\mathrm{Ni}$ atoms were O-terminated in the intersheet direction. The dimensions of the model fragments are defined as $\mathrm{X} \times \mathrm{Y}$ $\times \mathrm{Z}$, where $\mathrm{X}, \mathrm{Y}$ and $\mathrm{Z}$ are the number of intrasheet $(\mathrm{X}, \mathrm{Y})$, and intersheet $(\mathrm{Z})$ repeats of the brucite $\mathrm{Ni}(\mathrm{OH})_{2}$ unit cell. We assume for simplicity that the molecular fragments have $\mathrm{X}=\mathrm{Y}$. 
Figure 3.1 shows PDF patterns of $\mathrm{NiO}_{x} \mathrm{H}_{y}$ and $\mathrm{Ni}_{85} \mathrm{Fe}_{15} \mathrm{O}_{x} \mathrm{H}_{y}$ films and of their corresponding best-fit models. The $\mathrm{NiO}_{x} \mathrm{H}_{y}$ system (Fig 1a, top) is best modeled by a layered structure with the domain dimensions of $12 \times 12 \times 2$ unit cells. The $\mathrm{Ni}_{85} \mathrm{Fe}_{15} \mathrm{O}_{x} \mathrm{H}_{y}$ structure (Fig 3.1a, bottom) is best modeled by a single-sheet structure with the domain dimensions of $9 \times 9 \times 1$ unit cells. We caution the reader that the model PDF patterns shown in this chapter do not include an instrumental dampening correction (see Instrumental Dampening section) which may lead to an underestimation of domain size. We refer the reader to our manuscript for updated model refinement.

Since PDF measures atom-to-atom correlations, randomness introduced through translation or rotations of adjacent sheets would explain their lack of contribution to $\mathrm{G}(r)$ and thus the observation that only a single layer is needed to fit the PDF data in the case of $\mathrm{Ni}_{85} \mathrm{Fe}_{15} \mathrm{O}_{x} \mathrm{H}_{y}$. XRD can be a complimentary technique for determining intersheet distances. ${ }^{32}$ This is because the Bragg reflections of $<001>$ planes, requiring only a constant intersheet spacing, are not affected by translation or rotation of adjacent sheets. ${ }^{61}$ 

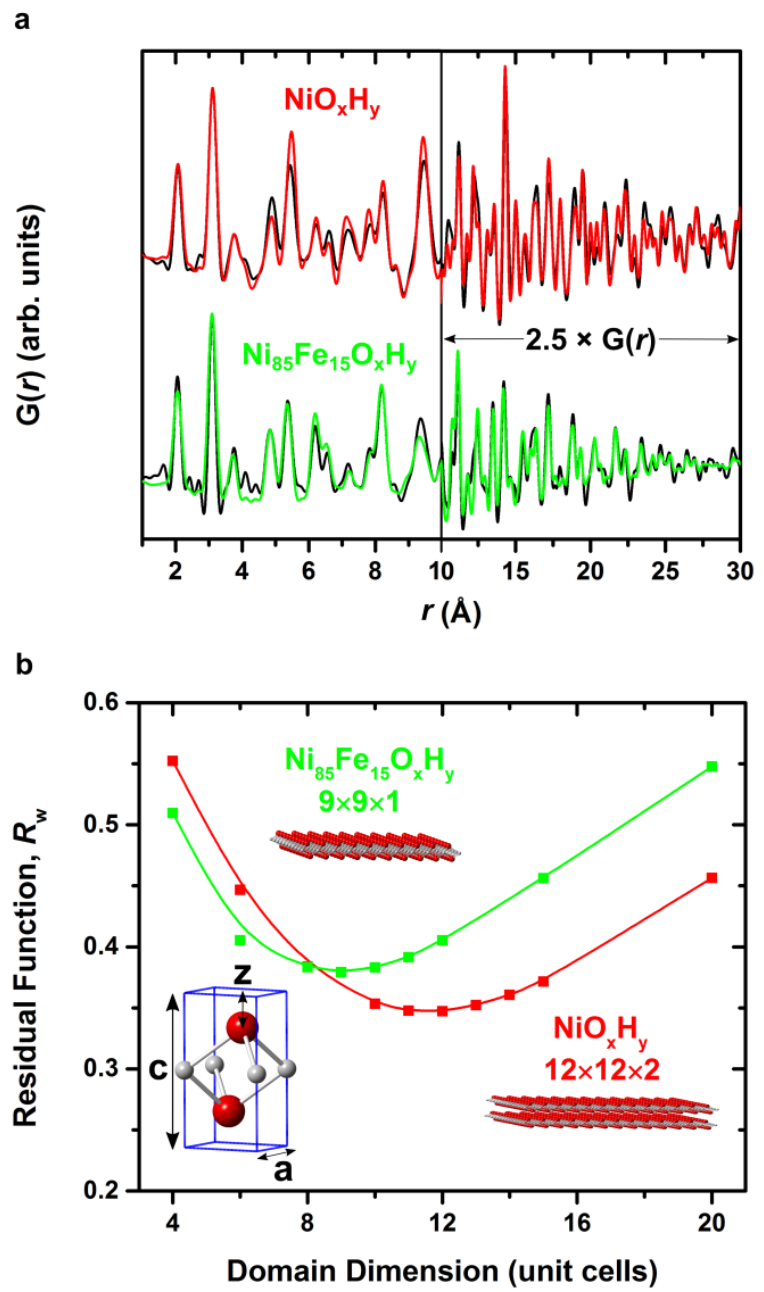

Figure 3.1. a) Experimental and model PDF patterns of (top) $\mathrm{NiO}_{x} \mathrm{H}_{y}$ and (bottom) $\mathrm{Ni}_{85} \mathrm{Fe}_{15} \mathrm{O}_{x} \mathrm{H}_{y}$ pulse-deposited films. Films shown were deposited and cycled once leaving them in a fully reduced state. The patterns (model and experimental) beyond 10 $\AA$ have been scaled to give the high $r$ peaks a similar intensity as the low $r$ peaks. In $b$ ), the residual function (i.e. goodness of fit) is plotted versus the number of unit cells contained in the model along the (100) and (010) directions. The models and unit cell are shown as insets. The $\mathrm{NiO}_{x} \mathrm{H}_{y}$ film matches well with a bilayer hydroxide domain. The $\mathrm{Ni}_{85} \mathrm{Fe}_{15} \mathrm{O}_{x} \mathrm{H}_{y}$ film is best represented by a smaller single-sheet domain. For limitations of the model patterns and domain sizes please see the Instrumental Dampening section.

While the models in Figure $1 \mathrm{~b}$ best match the entirety of the PDF data, there are certain $r$ ranges that are better fit with other domain sizes. Generally, the small $r$ range $(<$ $10 \AA$ ) is better fit by models with smaller dimensions, and high $r$ by models with larger 
dimensions. This is likely due to film heterogeneity, as discussed in the Film Heterogeneity Section.

\section{Electrochemical Cycling.}

Cyclic voltammetry is an essential tool for studying electrocatalysts. It can be used to follow the transformation from one morphology to another through the growth or shifting of redox features. Integration of those redox features can also provide a quantitative estimate of the electrochemically active surface area. ${ }^{19}$

One question is the degree to which electrochemical cycling from the resting (reduced hydroxide) to active (oxidized oxyhydroxide) state changes the intermediate structure of the (oxy)hydroxides and the degree to which this is important for the catalytic mechanism. To understand the effect of cycling on intermediate structure, we have performed ex situ scattering experiments and PDF analysis on as-deposited, oxidized, and reduced samples for (a) pulse-deposited $\mathrm{NiO}_{x} \mathrm{H}_{y}$ and (b) continuously deposited $\mathrm{Ni}_{77} \mathrm{Fe}_{23} \mathrm{O}_{x} \mathrm{H}_{y}$ films (Figure 3.2). These particular films were chosen for the following reasons. The pulse-deposited $\mathrm{NiO}_{x} \mathrm{H}_{y}$ is expected to be more homogeneous, denser, and better electrochemically interconnected than films deposited by continuous electrodeposition, as we have previously shown. ${ }^{55}$ The continuously deposited $\mathrm{Ni}_{77} \mathrm{Fe}_{23} \mathrm{O}_{x} \mathrm{H}_{y}$ films, in contrast, have been found to contain a non-homogenous Fe distribution and a more-open morphology that may be more amenable to structural rearrangement during cycling.

The results in Figure 3.2 show significant, reversible, redox-state-dependent changes in bond length for both the (a) pulse-deposited $\mathrm{NiO}_{x} \mathrm{H}_{y}$ and (b) continuously 
deposited $\mathrm{Ni}_{77} \mathrm{Fe}_{23} \mathrm{O}_{x} \mathrm{H}_{y}$ films. For example, the first shell $\mathrm{Ni}(\mathrm{Fe})-\mathrm{O}$ shortens from $2.1 \AA$ to $1.9 \AA$ upon oxidation, and accompanied by a corresponding decrease in the di- $\mu$-oxo linked $\mathrm{Ni}(\mathrm{Fe})-\mathrm{Ni}(\mathrm{Fe})$ distance from $3.1 \AA$ to $2.9 \AA$. In addition, reversible redox-state dependent changes are seen in the intermediate length scale, including the apparent shifts of PDF peaks at $5.4 \AA$ and $8.2 \AA$ in the reduced films to $4.9 \AA$ and $7.6 \AA$, respectively, in the oxidized films. These peaks correspond to predominantly Ni-Ni intrasheet distances and suggest redox-state dependent contraction and expansion of the individual sheets.

In both cases, however, redox-dependent structure changes are reversible, and the films show the absence of large, persistent changes in scattering patterns induced by electrochemical cycling, suggesting structural stability. This data is important, because it shows for both structures that the resting (i.e. reduced hydroxide) structure is conserved during electrochemical measurement at OER potentials and thus serves as a useful proxy for the local domain (sheet) size of the active catalyst phase. Further study on the stability of $\mathrm{Ni}_{(1-z)} \mathrm{Fe}_{z} \mathrm{O}_{x} \mathrm{H}_{y}$ films held at oxidizing potentials / higher current densities for longer periods is currently underway, and may shed light on how film structure changes influence the time-dependent OER performance of the films, ${ }^{62,63}$ which is of importance for applications. 

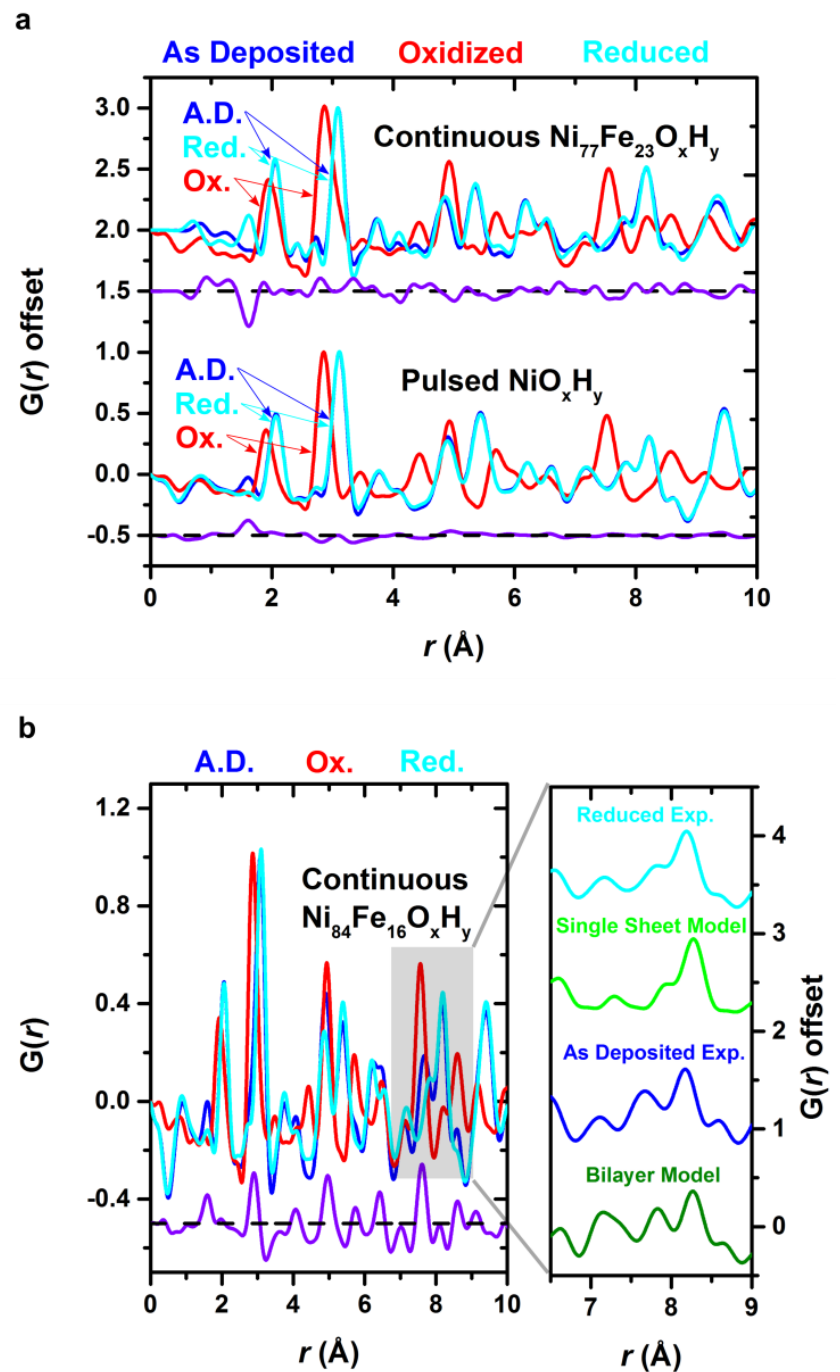

Figure 3.2. $\mathrm{PDF}$ patterns of a) $\mathrm{NiO}_{x} \mathrm{H}_{y}, \mathrm{Ni}_{77} \mathrm{Fe}_{23} \mathrm{O}_{x} \mathrm{H}_{y}$, and b) $\mathrm{Ni}_{84} \mathrm{Fe}_{16} \mathrm{O}_{x} \mathrm{H}_{y}$ films in three different states of conditioning: as deposited (blue), oxidized (red), and reduced (cyan). "Residuals" of as-deposited minus re-reduced experimental patterns are shown to illustrate small structural changes upon cycling and are centered at $\mathrm{G}(r)=-0.5$. The optical absorption changes indicate that the as-deposited and reduced films contain $\mathrm{Ni}$ in the $2^{+}$oxidation state versus (nominally) $3^{+} / 4^{+}$in the oxidized films. The $\mathrm{NiO}_{x} \mathrm{H}_{y}$ and $\mathrm{Ni}_{77} \mathrm{Fe}_{23} \mathrm{O}_{x} \mathrm{H}_{y}$ systems in a) match well with a bilayer hydroxide and a single-sheet structure, respectively, both before and after cycling. In $\mathrm{b}$, the as-deposited $\mathrm{Ni}_{84} \mathrm{Fe}_{16} \mathrm{O}_{x} \mathrm{H}_{y}$ film has a PDF pattern better fit by a bilayer structure, but the re-reduced film is better modeled as a single sheet. 
We also note that some of the systems studied here did show differences in the PDFs collected from the as-deposited and re-reduced states. For example, the as-deposited (continuous method) $\mathrm{Ni}_{84} \mathrm{Fe}_{16} \mathrm{O}_{x} \mathrm{H}_{y}$ is best modeled with a bilayer domain, but the intersheet interactions are not present in the re-reduced sample, which is best-modeled by a single-layer sheet domain. However, no changes in the domain size of the individual sheet are found. Generally, we observe that the pure $\mathrm{NiO}_{x} \mathrm{H}_{y}$ systems retain bilayer structures. Addition of large Fe fractions decreases the extent of ordered layering observable, consistent with our previous observation that the addition of $\mathrm{Fe}^{3+}$ to $\mathrm{Ni}(\mathrm{OH})_{2}$ increases the interlayer spacing due to charge-compensating anion intercalation which might weaken the non-covalent interactions between the sheets that lead to the characteristic features in the PDF associated with inter-sheet scattering.

\section{Bond Lengths.}

In addition to the intermediate-range structure, PDF analysis provides structural information on the first two coordination spheres which correspond to the metal-oxygen (M-O) and metal-oxygen-metal (M-O-M) bond lengths. Figure 3.3 shows the $\mathrm{M}-\mathrm{O}$ and M-O-M bond lengths for the catalysts in their nominally a) $\mathrm{Ni}^{2+}$, and b) $\mathrm{Ni}^{3+/ 4+}$ redox states, along with the FWHM of the PDF peaks associated with the M-O-M length. The addition of $\mathrm{Fe}$ into $\mathrm{Ni}(\mathrm{OH})_{2}$ decreases the average $\mathrm{M}-\mathrm{O}$ bond length, consistent with the substitution of $\mathrm{Fe}^{3+}$ onto $\mathrm{Ni}^{2+}$ sites in the structure. This behavior is consistent with the smaller effective radius of the $\mathrm{Fe}^{3+}$ ion compared to $\mathrm{Ni}^{2+}$ in the solid state, ${ }^{64}$ and that $\mathrm{Fe}$ substitutes for Ni in the (oxy)hydroxide sheet. We also note that the segregation of Fe-

rich phases has been reported for films with $>11 \% \mathrm{Fe},{ }^{34,65,66}$ although no such phase was 
evident from our PDF analysis. This does not preclude its presence, as it may be too minor of a component to contribute substantially to the PDF patterns.

Figure $3.3 \mathrm{~b}$ shows that the measured $\mathrm{M}-\mathrm{O}$ and $\mathrm{M}-\mathrm{O}-\mathrm{M}$ bond lengths in the oxidized $\mathrm{Ni}(\mathrm{Fe}) \mathrm{OOH}$ phase largely increase with Fe content. We caution, however, that this may be an artefact of measuring the samples ex situ where the amount of selfdischarge (i.e. the oxidized catalyst reacting with hydroxide electrolyte to return to the reduced state) is unknown. However, the FWHM of all the peaks are similar, and no significant shoulders in the PDF patterns were observed. These observations suggest that a large amount of self-discharge, which would result in mixed $\mathrm{Ni}^{2+}$ and $\mathrm{Ni}^{3+/ 4+}$ regions, did not occur. In situ X-ray absorption or total scattering experiments are needed to clarify this.

The bond lengths found here correspond well with values previously obtained through x-ray absorption fine structure (XAFS) measurements. ${ }^{34,38}$ Friebel et al. determined that the Ni-O and Ni-O-Ni distances were 2.06 and $3.10 \AA$, respectively, when the catalyst was in the reduced state. ${ }^{34} \mathrm{Fe}-\mathrm{O}$ bonds $(2.01 \AA)$ in $\mathrm{FeOOH}$ were determined to be shorter than $\mathrm{Ni}-\mathrm{O}$ in $\mathrm{NiOOH}$, which explain our observed decrease in $\mathrm{M}-\mathrm{O}$ bond length with increasing Fe content in $\mathrm{Ni}(\mathrm{Fe}) \mathrm{OOH}$. Friebel et al. also found an increase in $\mathrm{Fe}-\mathrm{O}$ bond length with increasing $\mathrm{Fe}$ content for oxidized films, though the effect was not as pronounced as in the data reported here for continuously deposited films. Bediako et al. found multiple Ni-O and Ni-O-Ni distances in their as-deposited films. ${ }^{38} \mathrm{Ni}-\mathrm{O}$ bond lengths were modeled at 1.9 and $2.10 \AA$, and $\mathrm{Ni}-\mathrm{Ni}$ bond lengths of 2.83 and $3.05 \AA$ best fit their data. A mixed valence of $\mathrm{Ni}^{2+}$ and $\mathrm{Ni}^{3+/ 4+}$ would explain this data as the longer bond lengths match well with the values measured here for the film 
in its fully reduced state, and the shorter with the bond lengths measured for our oxidized films.

a

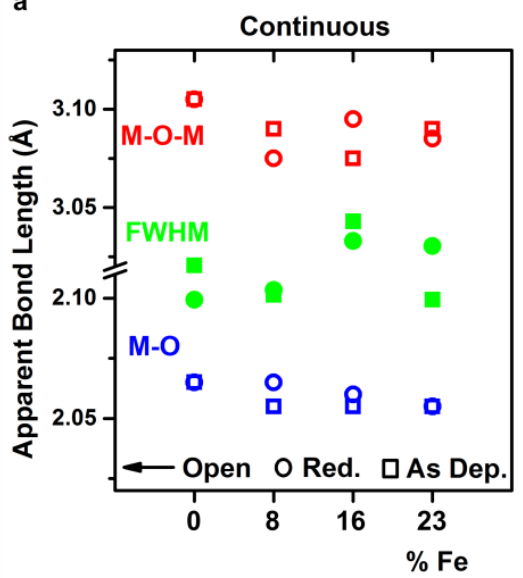

b

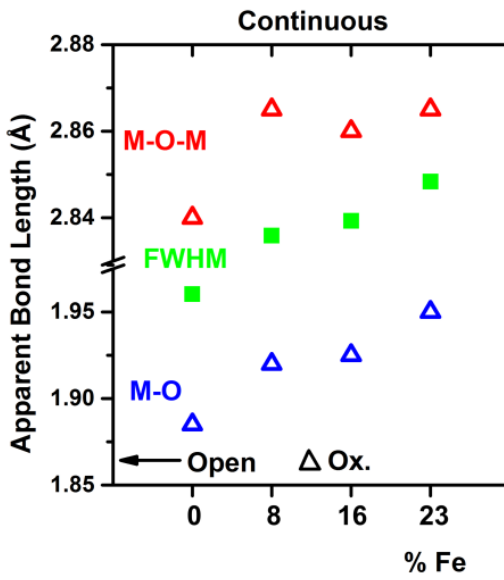

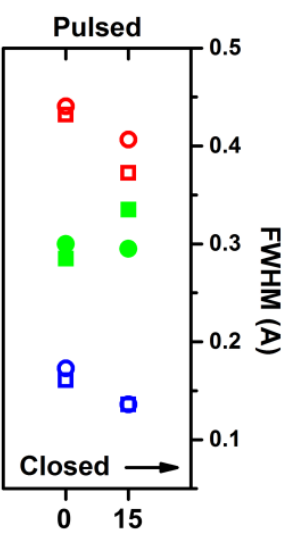

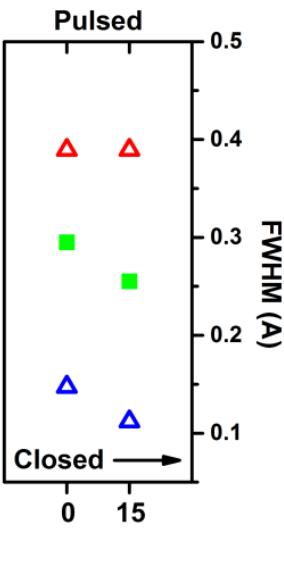

Figure 3.3. $\mathrm{M}-\mathrm{O}$ (bottom half) and $\mathrm{M}-\mathrm{O}-\mathrm{M}$ (top half) bond lengths for the films in nominally a) $\mathrm{Ni}^{2+}$ and b) $\mathrm{Ni}^{3+/ 4+}$ states, studied as a function of $\mathrm{Fe}$ content for continuous and pulse-deposited films. The squares/circles are for as-deposited and re-reduced films, respectively, while the triangles are for oxidized films. Each point represents only one sample, and therefore no error statistics are available.

\section{Effects of Pulsed Deposition on Morphology.}

Our previous work showed a significant improvement in oxygen evolution performance through the use of a pulsed electrodeposition, especially for films at high mass loadings (e.g. $\left.\sim 0.1 \mathrm{mg} \mathrm{cm}^{-2}\right) .{ }^{55}$ The enhanced activity was explained by the 
observation that the pulse-deposited films were denser (as seen by SEM imaging) and better electrically interconnected (as evident from increased electrochemical charge reversibility). ${ }^{55}$

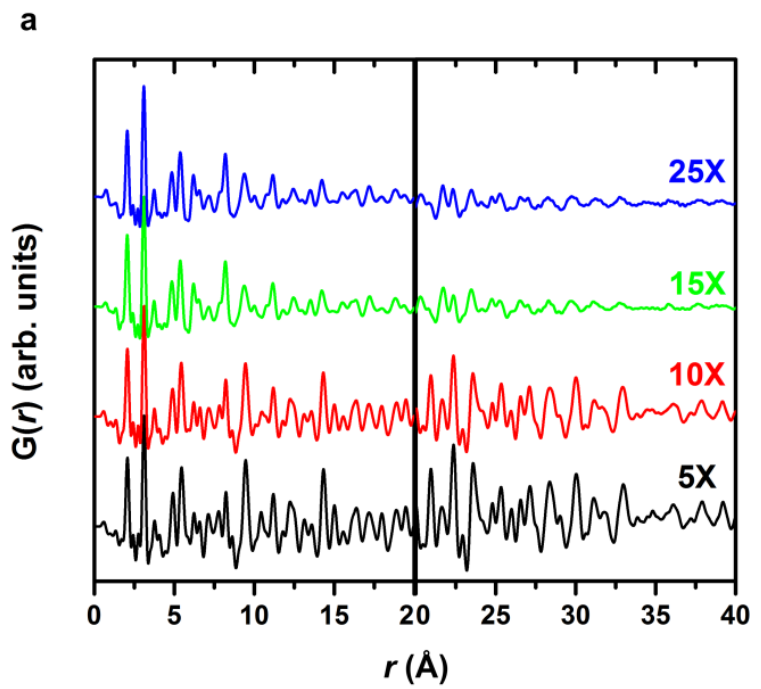

b

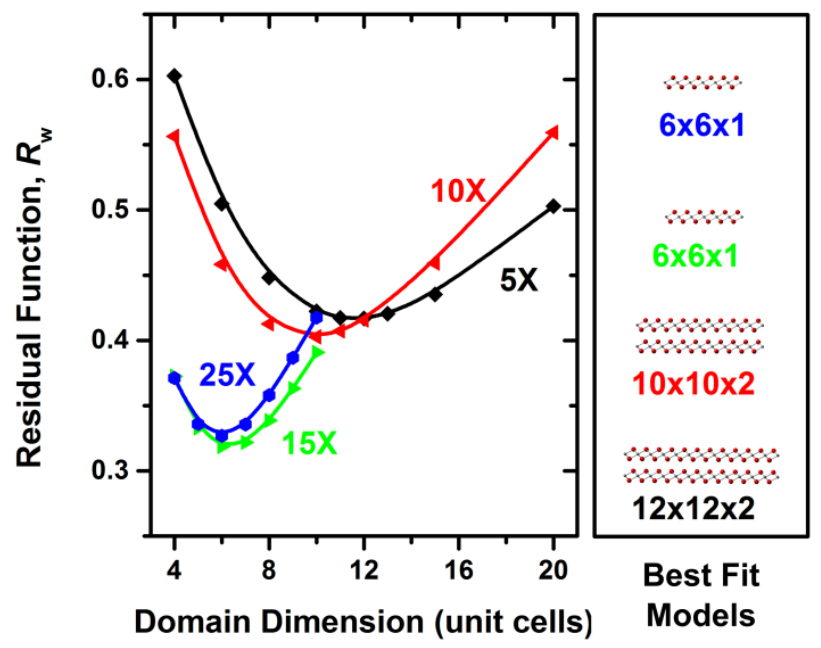

Figure 3.4. Effect of the number of deposition pulses on the intermediate structure of $\mathrm{NiO}_{x} \mathrm{H}_{y}$ films. A) PDF patterns for catalysts deposited using 5, 10, 15, and 25 repeats of 2 $\mathrm{s}$ cathodic current pulses. B) Fit values as a function of domain dimension for each of the films shown in a). The patterns for films from 5 and 10 pulses are best fit by bi-layered structures while those with 15 and 25 pulses are best fit by single sheet structures. While the films synthesized with 15 and 25 pulses are best-fit by the same simulated structure, variations in their residual function show that they are not identical, and require a morecomplex model for differentiation. For limitations of the model patterns and domain sizes please see the Instrumental Dampening section. 
To better understand the pulsed electrodeposition process, we measured PDFs for $\mathrm{NiO}_{x} \mathrm{H}_{y}$ as a function of the number of deposition pulses (Figure 3.4). A decrease in coherence length with increasing number of deposition pulses is evident from the faster decay in pair correlation intensity with $r$ for larger pulse numbers. For example, the $\mathrm{NiO}_{x} \mathrm{H}_{y}$ film prepared with five pulses is best described by a $12 \times 12 \times 2$ bilayer model, while the film prepared with 25 pulses is best modeled with a $6 \times 6 \times 1$ single layer. The presence of smaller domains may contribute, in part, to the higher intrinsic activity of pulse-deposited films,${ }^{55}$ as Fe edge sites have been predicted to have high OER activity in $\mathrm{Ni}(\mathrm{Fe}) \mathrm{O}_{x} \mathrm{H}_{y}$ films. ${ }^{34}$ This reduction in coherence length appears to be unique to the high number of pulses, as neither Fe content nor film conditioning had such an effect.

The reduction in coherence length can be understood through the physiochemical processes occurring during deposition. Cathodic electrodeposition from aq. nitrate salts proceeds through a reduction of nitrates. One possible reaction is: ${ }^{13}$

$$
\mathrm{NO}_{3}^{-}+7 \mathrm{H}_{2} \mathrm{O}+8 \mathrm{e}^{-} \rightarrow \mathrm{NH}_{4}^{+}+10 \mathrm{OH}^{-}
$$

The local $\mathrm{pH}$ increase results in the precipitation of nominally $\mathrm{Ni}(\mathrm{Fe})(\mathrm{OH})_{2}$ (other electrolyte ions are also likely included with the interlayer structure). The use of short electrochemical pulses with intervening periods of mechanical stirring: 1) allows the local $\mathrm{pH}$ to decrease when a potential is not being applied, possibly causing etching and partial dissolution of the deposited film, and 2) allows time for fresh electrolyte move into the pores of previously deposited layers, which then deposit into increasingly smaller spaces with each consecutive pulse and reducing the average coherence length of the (oxy)hydroxide sheets. Because the nitrate reduction reaction proceeds on the conductive 
electrode surface (and not on the deposited catalyst, which is an insulator under cathodic potentials) ${ }^{33}$ the pulsed deposition infills and creates a denser film structure.

\section{Film Heterogeneity.}

In order to identify other possible sources of heterogeneity in the intermediate structures, we created more-complicated structural models that incorporate mixed populations of nanosheet sizes and different numbers of layers. We also investigated the effect of both $\mathrm{Ni}$ and $\mathrm{O}$ vacancies as was done by Abidat et al. ${ }^{67}$ We then analyzed how each of these affects the residual error between experiment and the model data (Figure 3.5). For simplicity we applied the more-complicated model structure only to the $\mathrm{Ni}(\mathrm{OH})_{2}$ film deposited with five pulses (i.e. this data was previously shown in Figure 3.4a). 

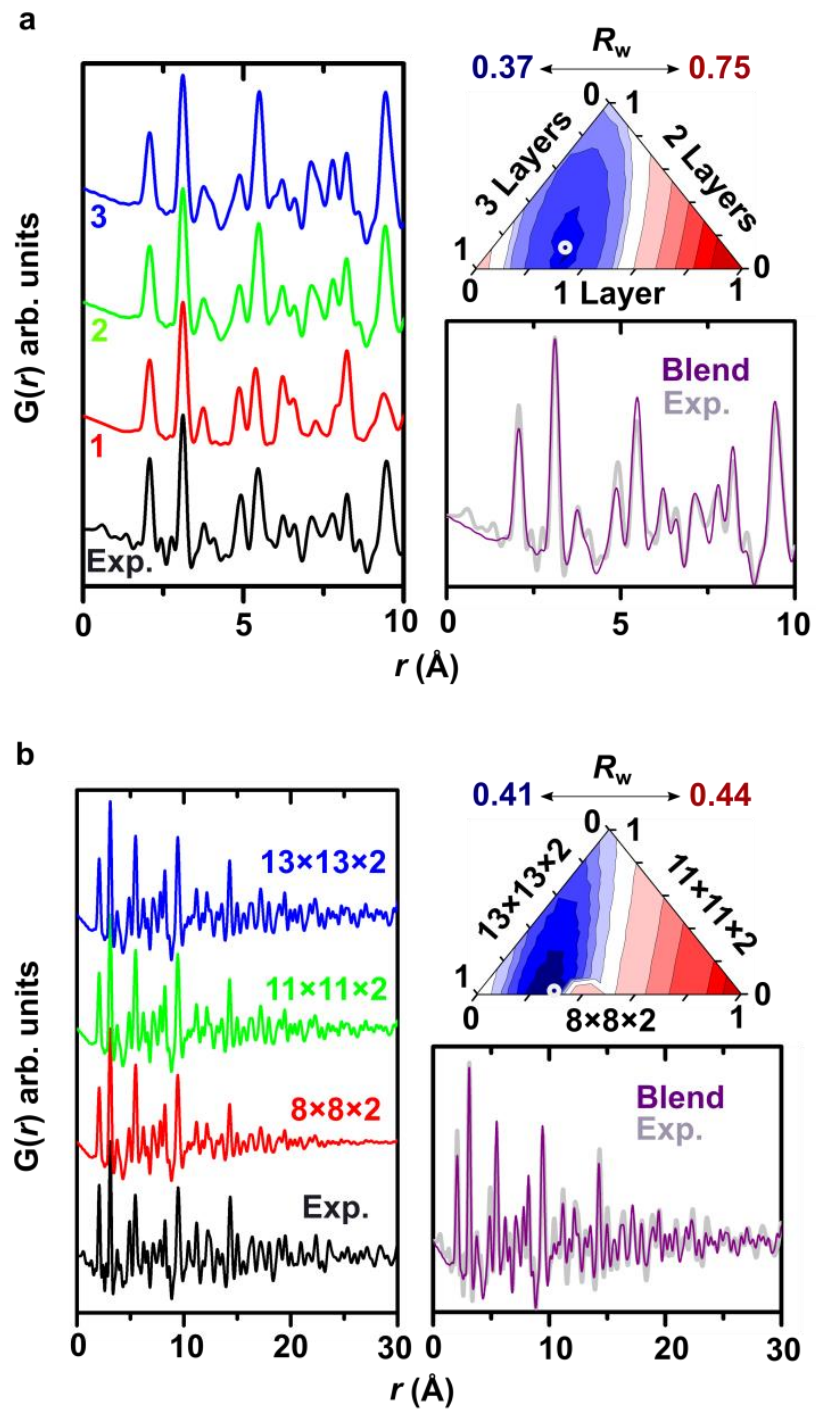

Figure 3.5. Heterogeneity in the $\mathrm{NiO}_{x} \mathrm{H}_{y}$ film with 5 pulses shown in Fig 3.4. Parameters varied include a) number of coherent layers in domain and b) domain dimension. Offset experimental and model PDF patterns (left) are shown with ternary diagrams (upper right) highlighting the optimal model for a linear combination of the three components. Overlaid PDF patterns of the experimental (grey) and optimized model blend (purple) are shown in the bottom right of both panels. For limitations of the model patterns and domain sizes please see the Instrumental Dampening section.

We first studied the degree to which heterogeneity in layering affected the residual error $R_{\mathrm{w}}$. As shown in Figure 3.4b, the best fit for a homogeneous model (for the sample with five pulses) was given by the $12 \times 12 \times 2$ model which had $R_{\mathrm{w}}=0.42$. In 
Figure 3.5a the PDF patterns for mono-, bi- and tri-layered $12 \times 12$ models are shown. By using linear combination of these models we are able to decrease $R_{\mathrm{w}}$ by $>10 \%$. The improved fitting is primarily due to better fitting the peaks in the low $r$ region between 1.8 and $10 \AA$, and suggest that the samples showing a "bilayer structure" indeed include significant single and trilayer domains.

We also noticed that a single domain-size model does not accurately simulate the PDF intensity profile over all $r$, indicating that there must be different sized domains contributing to the PDF intensity. To analyze such size heterogeneity we constructed a model with three different components (Figure $3.5 b$ ); an $8 \times 8$ model that best fit experimental PDF data below $10 \AA$, an $11 \times 11$ model that best fit the experimental data from $20-30 \AA$, and a $13 \times 13$ model that best fit the experimental data from $30-50 \AA$. The best-fit combination favors the $13 \times 13$ model, consistent with the large range of $r$ that is best fit by that domain size. The narrow $R_{\mathrm{w}}$ range produced by varying domain size, in contrast to varying layers, is due to the similarity of model PDF patterns in the low $r$ region.

\section{Instrumental Dampening.}

All of the model PDF patterns displayed in this chapter show decay that comes primarily from their relative sizes and does not include the instrumental dampening that contributes to the decay of the experimental PDF patterns. To address this, PDFGui was used to obtain a dampening value of 0.045 for the $\mathrm{CeO}_{2}$ standard under our experimental conditions. Figure 3.6 shows this dampening function overlayed with the PDF pattern of the $\mathrm{CeO}_{2}$ standard. 


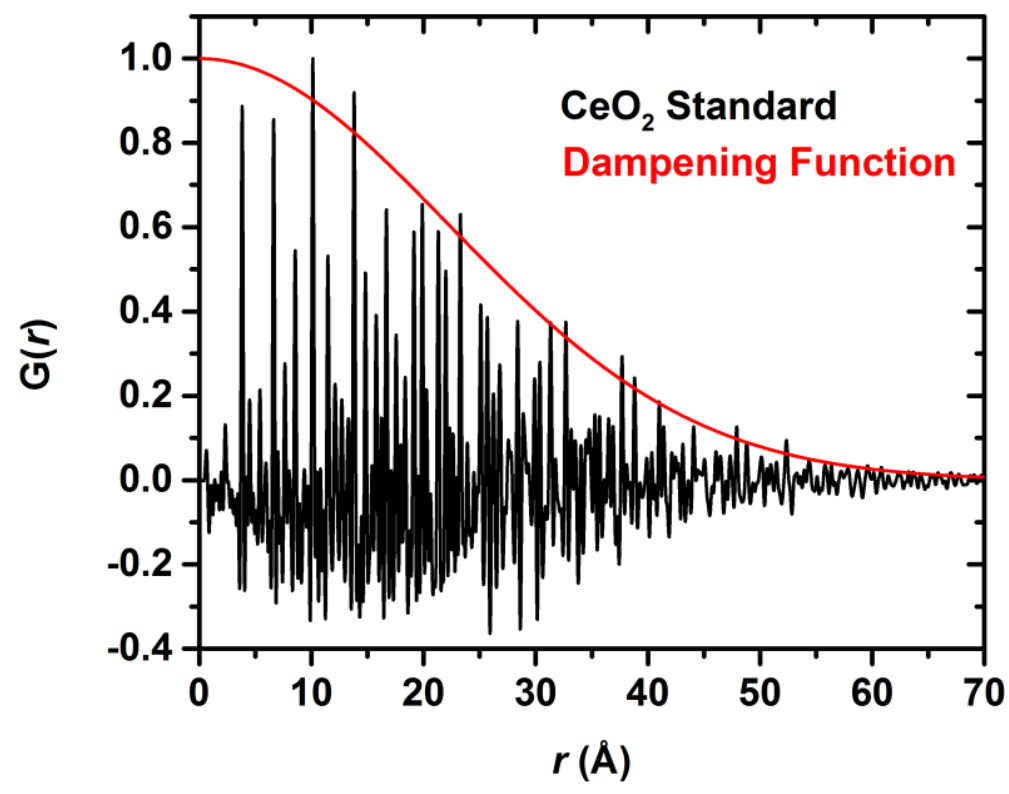

Figure 3.6. PDF pattern of the $\mathrm{CeO}_{2}$ standard and the instrumental dampening function that will be used to correct the model PDF patterns shown in this chapter.

The model PDF patterns, in the absence of this dampening function, have greater intensity in the high $\mathrm{r}$ region than they would if collected under our experimental conditions. It is therefore possible that the values of domain size reported to best describe the experimental data are smaller than the true value. This instrumental dampening will disproportionately affect the larger models that display high intensities at high $r$ where the effects of the Gaussian function are more pronounced. We point the readers of this dissertation to our published work to find the more accurate structural models.

It is important to point out that all claims of relative size in this chapter are still valid. The experimental pair distribution patterns already contain contributions from the instrumental dampening and so the intensities at high $r$ values can be considered real and do not require further corrections. 


\section{Conclusion and Bridge}

We report the first PDF analysis on $\mathrm{Ni}_{(1-z)} \mathrm{Fe}_{z} \mathrm{O}_{x} \mathrm{H}_{y}$ OER catalysts. The nanosheet oxyhydroxide domains that make up the films and are well-modeled by a brucite-like $\mathrm{Ni}(\mathrm{OH})_{2}$ unit cell. The bond lengths of the reduced films agree with literature values for both Fe-free and $\mathrm{Ni}_{(1-z)} \mathrm{Fe}_{z} \mathrm{O}_{x} \mathrm{H}_{y}$ materials. The films show minimal loss in intrasheet coherence following electrochemical cycling, indicating structural stability. While no significant difference between the domain size of pulsed and continuously deposited films were observed for films made with $<10$ pulses, nor for increasing Fe content, increasing the number of pulses reduces the size of the coherent scattering domain and thus forms denser catalyst layers with more nanosheet edge sites. The findings here represent a foundation upon which future structural studies can be compared, highlighting differences in the intermediate structure of $\mathrm{Ni}(\mathrm{Fe}) \mathrm{OOH}$-based catalysts due to variations in synthesis, Fe content, and electrochemical treatment.

In Chapter III we learned that the local environments (unit cell) of the pulse and continuously deposited films are nominally the same, and thus not the source of the significant difference in the loading-dependent activity shown in Chapter II. This points to the source of the variable activities extending beyond the local, and perhaps even intermediate, domain lengths within the larger film architecture. We are thus able to turn our attention towards optimizing the bulk properties of the film, which is the focus of Chapter IV. 


\section{CHAPTER IV}

\section{OPTIMIZATION OF PULSED ELECTRODEPOSITIONS FOR HIGH PERFORMING $\mathrm{NIFEO}_{X} \mathrm{H}_{Y}$ FILMS}

This section contains no previously published or coauthored work

\section{Introduction}

Water splitting has a greater than 200 year history, with as many as 400 industrial electrolyzers in operation in $1900 .{ }^{1}$ Unfortunately, the inefficiency towards the OER has hindered its contemporary growth. ${ }^{2,3}$ Efforts towards understanding and lowering the overpotential associated with the chemical activation $\eta_{\text {act }}$ of OER have been ongoing for several decades. ${ }^{4}$ In acid, Ru and Ir oxides have been the standard upon which all catalysts have been compared. ${ }^{5}$ In base, Fe-doped Ni and Co oxyhydroxide films have shown to outperform these catalysts, ${ }^{6}$ and the former has become the standard upon which all OER catalysts are compared, even though a broad range of $\mathrm{Ni}$ :Fe ratios have been reported to be the most active. ${ }^{7-9}$ Binary challengers to NiFe-based catalysts seem unlikely at this point, but the use of high throughput screening has yielded exciting new ternary and quaternary combinations of transition metals worthy of further exploration. ${ }^{10-}$ 12

Finding an ideal chemical composition is an important stepping stone on the way to an efficient water splitting device, as the orders of magnitude difference in activity for different systems demonstrates. ${ }^{13}$ However, it is only one contribution to the overall 
overpotential of the system. ${ }^{14,15}$ Alkaline electrolyzers, seeking to achieve current densities on the order of $500 \mathrm{~mA} \mathrm{~cm}{ }^{-2}$ or greater, ${ }^{16}$ necessitate the deposition of high loading films in order to minimize the energy inputs of the system. ${ }^{17}$ Barring order of magnitude improvements in chemical activation, loadings on the order of tens of $\mathrm{mg} \mathrm{cm}$ will be required. ${ }^{18}$ Based on reported interlayer distances for NiFe films, ${ }^{19}$ this is equivalent thicknesses approaching hundreds of microns an to over 100,000 layers.

There are several methods that can be employed to reduce mass transport within such thick films. Largely these fall into two categories. The first is through the use of OER inert substrates with a high surface area upon which the catalyst can be deposited. The second is the creation of channels within the catalyst material through the use of sacrificial templates.

Common materials used as inert supports are Ni mesh and foam, which both provide a higher surface to volume ratio compared to traditional planar electrodes. ${ }^{20-22}$ This allows for the reduction of both mass and charge transport as the percentage of catalyst in contact with the high conductivity electrode increases and the decrease in relative volume limits the mass diffusion length. Carbon based substrates have also been employed to include nanotubes ${ }^{23}$ and graphene sheets ${ }^{24}$. The nanotubes provide both high surface area and improved electronic wiring of the catalyst throughout the bulk of the film. The planar nature of the graphene may not increase the surface area of the catalyst, but if films are assembled through an alternating catalyst:graphene stacking sequence, better bulk conductivity may be achieved. ${ }^{24}$ The drawback to the use of inert substrates is that it adds to the volume and cost of the combined catalyst-substrate electrode material without providing a directly contributing to the OER. 
The unique thermodynamic stabilities of different materials under different conditions can be exploited through the use of a sacrificial template which dissolves away leaving the desired catalyst morphology behind. In Ni films, this has traditionally been accomplished through the deposition of "Raney" Ni hydroxide which contains Al or $\mathrm{Zn}^{25,26}$ Introduction of the bimetallic film into an alkaline solution results in $\mathrm{Al}$ or $\mathrm{Zn}$ dissolution, leaving a porous Ni framework behind. A similar templating can be achieved through the use of polystyrene beads, which can then be removed by a toluene treatment, leaving well defined, micron size pores,${ }^{27}$ or high surface area spheres. ${ }^{28}$

An under investigated area is understanding how different deposition conditions control film morphologies and performance ${ }^{29}$, and whether inert supports or sacrificial templates are even required. The conditions under which OER catalyst films are deposited are vast. The Jaramillo group attempted to synthesize a wide variety of OER catalysts using previously published conditions. ${ }^{30}$ These conditions spanned 50 fold differences in deposition current density and more than two orders of magnitude differences in deposition time. While this likely ensured that each of the films could be synthesized successfully, it serves to highlight a common experimental and conceptual oversight: that regardless of the deposition conditions, films of equivalent stoichiometry, in the same electrolyte, given sufficient time, are the same. This confuses the thermodynamic argument of a material tending towards its most stable phase, with nonthermodynamically controlled features of a film like porosity, dopant distribution, and surface roughness.

Here we report the optimization of a pulsed deposition technique that has previously been shown to improve the transport properties of a film without the use of 
either inert substrates or sacrificial templates. ${ }^{31}$ We find that of the variables investigated, the deposition current density has the largest effect on film performance. Turnover frequencies are improved by more than a factor of 2 over the unoptimized systems, and current densities exceeding those of industrial alkaline electrolyzers are reached at overpotentials below $400 \mathrm{mV}$.

\section{Experimental}

\section{Electrode Fabrication.}

Conductive Au-coated electrode substrates were prepared by first cleaning glass slides in a 3:1 hydrogen peroxide to sulfuric acid (piranha) solution followed by plasma etching. Next, $10 \mathrm{~nm}$ of $\mathrm{Ti}$ and $100 \mathrm{~nm}$ of $\mathrm{Au}$ were deposited by electron beam evaporation. glass slides were cut into $\sim 0.15-0.25 \mathrm{~cm}^{2}$ squares. $\mathrm{Cu}$ wire was attached to the slides via Ag paint. Exposed wire and Ag paint were sealed with epoxy (Hysol 1C). Au QCM slides were purchased from Stanford Research Systems.

\section{Film Deposition.}

Prior to film deposition, the Au and FTO electrodes were cycled twice in $1 \mathrm{M}$ $\mathrm{KOH}$ from 0.0 to $0.65 \mathrm{~V}$ vs. $\mathrm{Hg} / \mathrm{HgO}$. The deposition cell used a two electrode configuration with carbon cloth serving as the counter electrode. The films were cathodically deposited from a $0.1 \mathrm{M}$ solution prepared by mixing $\mathrm{Ni}\left(\mathrm{NO}_{3}\right)_{2} \bullet 6 \mathrm{H}_{2} \mathrm{O}($ Alfa Aesar) and $\mathrm{FeCl}_{2} \bullet 4 \mathrm{H}_{2} \mathrm{O}$ (Sigma Aldrich) in a 90:10 (mol:mol) ratio with ultrapure 18.2 $\mathrm{M} \Omega \mathrm{cm}$ water as the solvent. The deposition solution was sparged with $\mathrm{N}_{2}$ for $20 \mathrm{~min}$ 
prior to film deposition. For the design of experiments (DOE) investigation, the cathodic current density, pulse duration and stir time were varied between -1 and $-200 \mathrm{~mA} \mathrm{~cm}{ }^{-2}$, 0.1 and 2 s, and 1 and 30s, respectively. Following the DOE, the cathodic current density was varied between -25 and $-200 \mathrm{~mA} \mathrm{~cm}^{-2}$, with the pulse duration and stir time kept constant at $0.2 \mathrm{~s}$, and $30 \mathrm{~s}$, respectively. All films were briefly dipped into ultrapure water prior to being submerged in the electrochemical cell to remove residual metal ions.

\section{Electrochemical Measurements.}

The electrochemical cell was set up in a three electrode configuration with a Pt counter electrode and a $1 \mathrm{M} \mathrm{KOH} \mathrm{Hg/HgO} \mathrm{reference} \mathrm{electrode.} \mathrm{The} \mathrm{polypropylene} \mathrm{cell}$ contained a 1.0 M solution of semiconductor-grade KOH (Sigma Aldrich) prepared with 18.2 $\mathrm{M} \Omega \mathrm{cm}$ water. The electrochemical cell was sparged continuously with $\mathrm{O}_{2}$ and magnetically stirred to dislodge bubbles that might accumulate on the electrode surface. Measurements were taken on a Biologic SP-200 potentiostat. Electrochemical data were corrected for the uncompensated series resistance $R_{\mathrm{u}}$, which was determined through fitting of AC impedance data to a modified Randles circuit. The range of $R_{\mathrm{u}}$ was 1-6 $\Omega$. The overpotential was determined by $\eta=E_{\mathrm{app}}-E^{\mathrm{o}}-i R_{\mathrm{u}}$.

\section{Film Loading and TOF Calculations.}

All film loadings reported were obtained through the integration of the (nominally) $\mathrm{Ni}^{2+/ 3+}$ anodic redox peak of the fully reduced film. To avoid any irregularities associated with the freshly deposited film, two CV cycles were used to equilibrate the film with the solution in the electrochemical cell prior to taking any 
electrochemical measurements. To return films to their fully reduced initial state, the partially oxidized films were held at reducing potentials (up to $-1.0 \mathrm{~V}$ vs $\mathrm{Hg} / \mathrm{HgO}$ ) until all the dark color associated with the oxidized films disappeared $(<20 \mathrm{~min}$ for the thickest films). No large cathodic reduction waves were observed which indicates that Ni ions were not reduced to metallic Ni.

Loadings were calculated using the ECSA as determined through the double layer capacitance as described in section 2.5. This is due to a non-trivial fraction of the $\mathrm{Ni}$ redox wave overlapping with OER. All TOF data were acquired through chronoamperometry with an $i R_{\mathrm{u}}$ correction applied and the number of metal sites taken from the ECSA.

Double Layer Capacitance.

AC impedance measurements were taken over the frequency range of $0.1 \mathrm{~Hz}$ to $100 \mathrm{kHz}$. Impedance measurements on charged films were taken at $0.6 \mathrm{~V}$ vs. $\mathrm{Hg} / \mathrm{HgO}$ and on uncharged films at either $-0.05 \mathrm{~V}$ or $0.05 \mathrm{~V}$ vs. $\mathrm{Hg} / \mathrm{HgO}$ for FTO and $\mathrm{Au}$ electrodes, respectively. Capacitance values were then obtained through fitting (EC Lab) using a modified Randles circuit containing a resistor $\left(R_{\mathrm{u}}\right)$ connected in series to a resistor (charge transfer resistance $R_{\mathrm{CT}}$ ) and a capacitor (double layer capacitance $C_{\mathrm{DL}}$ ) that were connected in parallel.

\section{Results and Discussion}

In Figure 4.1, the reversibility of the films deposited under different current densities is determined. In all cases an initial increase in reversibility is found, which 
then reaches a maximum, followed by a decline. This Figure highlights two important points: 1) that the reversibility cannot be improved indefinitely, and 2) that the film performance is governed by (at least) two competing processes.

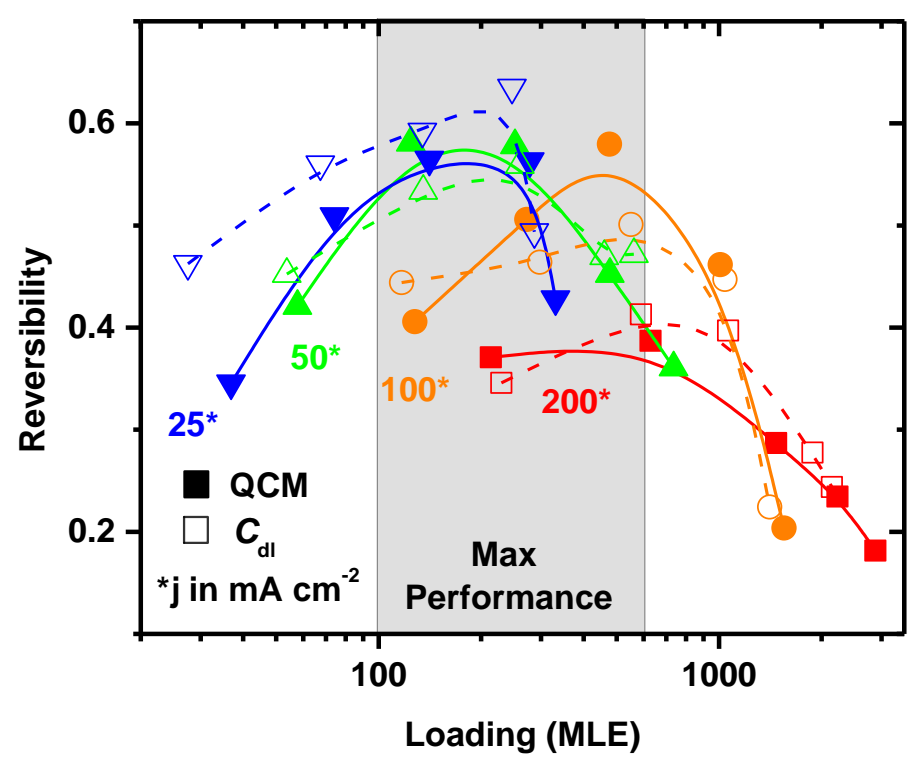

Figure 4.1. Reversibility as a function of loading for four different deposition current densities. Each $0.2 \mathrm{~s}$ deposition pulse was followed by $30 \mathrm{~s}$ of stirring. The amount of material participating in the reaction was determined through both the ECSA (open squares) as measured through impedance spectroscopy as well as through QCM mass measurements (closed squares). The grey region, spanning nearly 500 MLEs, seems to represent a range of loading where the reversibility is maximized for the different systems.

There is a well-known phenomenon in OER catalysis known as the Sabatier principle, ${ }^{32}$ or perhaps more descriptively, the volcano plot. This is often plotted to display a performance metric as a function of some descriptor such as oxygen binding strength. ${ }^{5,33-35}$ The idea being that there is some goldilocks region in a trade-off between two competing processes, which cannot be tuned independently. Like oxygen binding, transport processes should fall victim to the same goldilocks problem. Electron 
movement should benefit from a dense, highly interconnected film. Conversely, the movement of charge compensating ions, reactants and products is more easily accomplished in an open, porous network. It may also be worth noting that the high current densities reach their maximum reversibility sooner, and that the reversibility tends to decrease with increasing current density.

Previously, it has been shown that reversibility and activity are correlated. ${ }^{31}$ Figure 4.2 shows the TOF of the films from Figure 4.1.

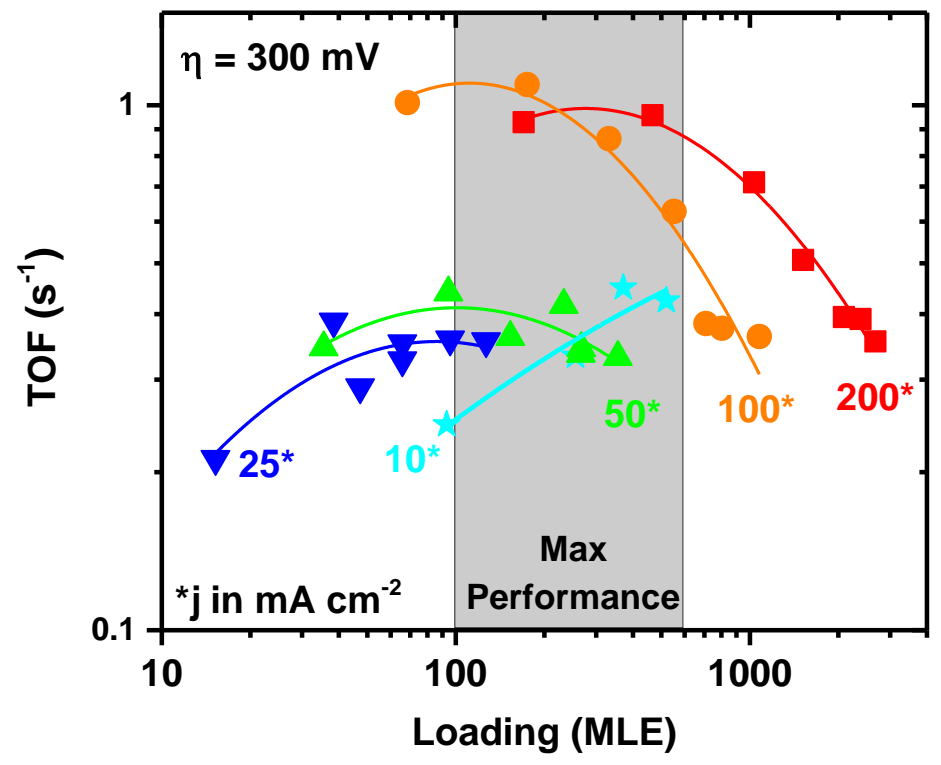

Figure 4.2. $\mathrm{TOF}$ at $300 \mathrm{mV}$ overpotential as a function of loading for four different deposition current densities. Each $0.2 \mathrm{~s}$ deposition pulse was followed by $30 \mathrm{~s}$ of stirring. The amount of material participating in the reaction was determined through the ECSA as measured through impedance spectroscopy. The grey region, spanning nearly 500 MLEs, seems to represent a range of loading where the TOF is maximized for the different systems. The data from our previous work ${ }^{31}$ (light blue) is included for comparison.

Figure 4.2 confirms that for films deposited under all four current densities, the loadings at which the highest reversibilities were found coincide with the highest TOF values. Surprisingly, however, the higher relative reversibilities of the lower deposition current 
densities did not result in the highest TOF. Instead, the high deposition current densities were shown to outperform the lower deposition current densities at equal loadings.

In the absence of $\eta_{\mathrm{mt}}$ and $\eta_{\mathrm{ct}}$, film loading could be increased indefinitely, with each active site having an equal TOF, due to the equal thermodynamic driving force needed, and felt, at all locations in the film. In this scenario, the current densities achievable would be limited by only the volumetric constraints of the device.

As the loadings of films are increased, the overpotential associated with mass transport $\eta_{\mathrm{mt}}$ may increase substantially. The $\eta_{\mathrm{mt}}$ arises from two phenomena 1 ) the deviation from bulk concentrations of the reactants and products, and 2) the blocking of active sites by generated $\mathrm{O}_{2}$ bubbles. As the concentration gradients begin to form, an increase in the thermodynamic driving force is necessary to continue to drive the reaction away from equilibrium, as described by the Nernst equation. Mechanical agitation in the form of stirring can help mitigate these two challenges by using convection to increase the flux of reactants in and products out, as well as in aiding the mechanical dislodgement of bubbles bound to the electrode surface. An inability to remove products, or reintroduce reactants throughout the film, could result in the transformation of the material from one demonstrating bulk activity to one of surface activity.

Charge transport is more complicated. It is hypothesized that pulsed depositions help to create a more interconnected film, which would improve the electron transport through the catalyst as the loadings are increased. What is not known is whether this actually minimizes the resistive loses due to a decrease in the number of "grain boundaries" between the platelet-like nanocrystalline regions of the film, or actually 
reduces the total resistance of the film by essentially wiring the catalyst in parallel instead of in series.

So far, the performance of the films has been shown to reach a maximum at a loading where mass and charge transport are well balanced. If mass transport is limiting, the value of this ideal loading should decrease at higher overpotentials, when the demands on mass transport and are increased. If charge transport is limiting, the opposite should be true.

Figure 4.3 shows that it is mass transport that limits film performance, with maximum activity reached at the lowest levels of loading.

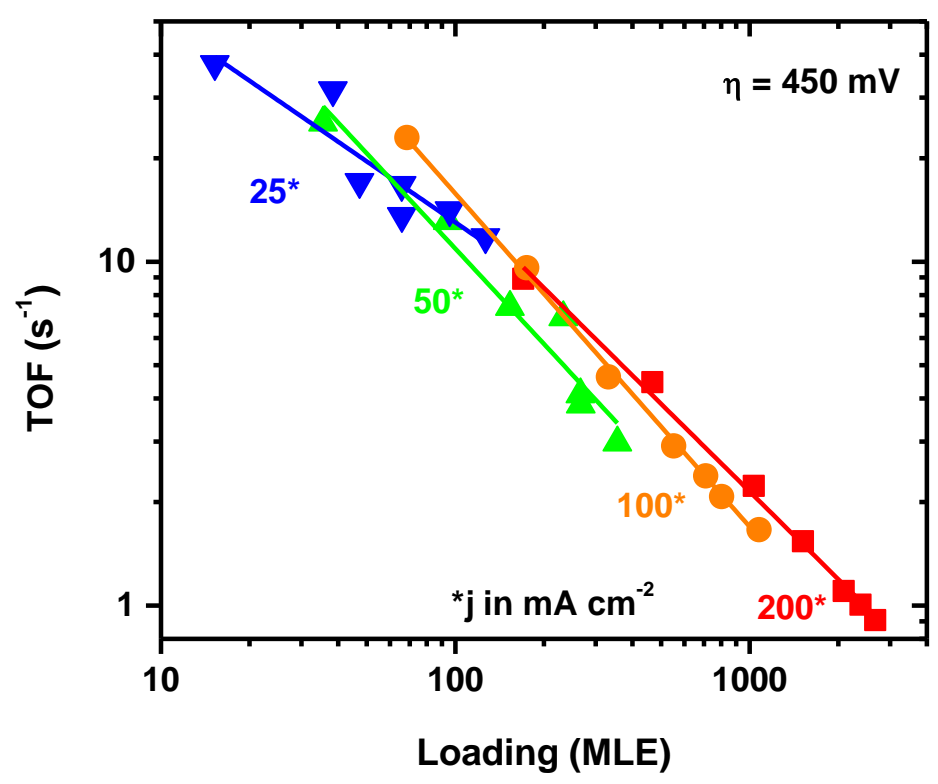

Figure 4.3. TOF at $450 \mathrm{mV}$ overpotential as a function of loading for four different deposition current densities. Each $0.2 \mathrm{~s}$ deposition pulse was followed by $30 \mathrm{~s}$ of stirring. The amount of material participating in the reaction was determined through the ECSA as measured through impedance spectroscopy.

The behavior of these films at $450 \mathrm{mV}$ overpotential is completely different than their behavior at $300 \mathrm{mV}$. At all loadings, there is an increase in TOF over those at $300 \mathrm{mv}$ 
overpotential as expected for the greater driving force. However, with Tafel slopes of between 30 and $40 \mathrm{mV} \mathrm{dec}^{-1}$, we would expect to see at least a four order of magnitude increase in TOF. For the thinnest films displayed in Fig 4.3, a greater than two order of magnitude increase is observed, however, for the thickest films, not even a factor of two improvement is reached.

The nearly linear decrease in TOF with loading could indicate that the films are transitioning from bulk or volume activity to one in which only a limited number of nearsurface active sites are participating. This is better analyzed by plotting the current as a function of loading.

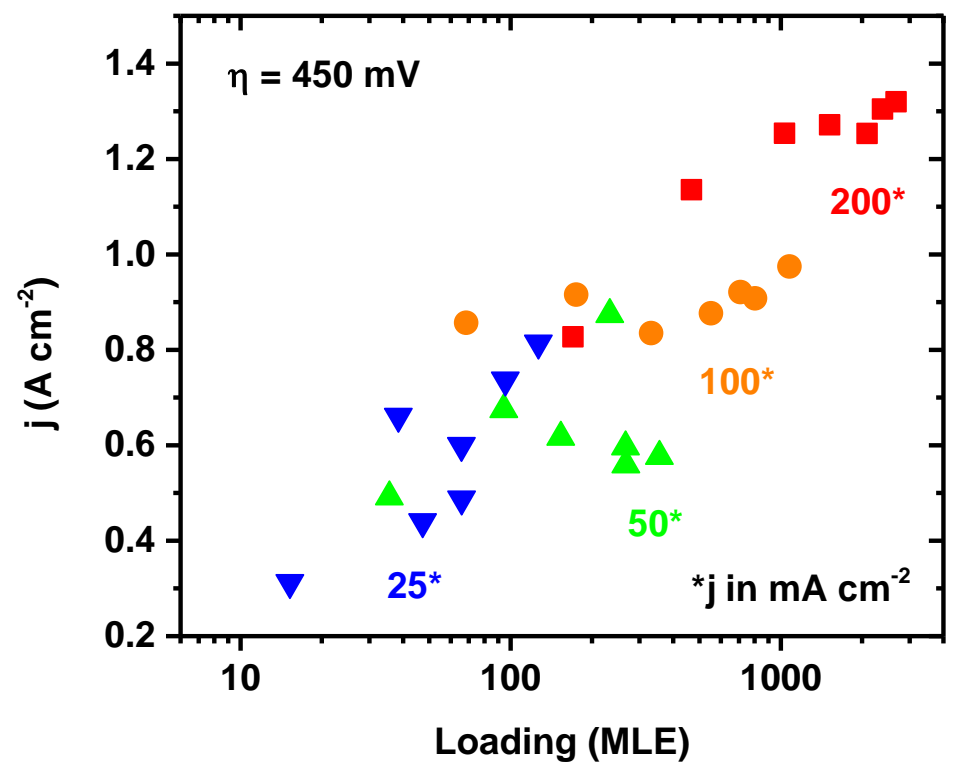

Figure 4.4. Current as a function of loading for the films in Figs. 4.2 and 4.3. Each $0.2 \mathrm{~s}$ deposition pulse was followed by $30 \mathrm{~s}$ of stirring. The loading was determined from the ECSA as measured by impedance spectroscopy. 
What can be understood from viewing the information in this way is that for the 3 higher deposition current densities, a maximum achievable current seems to be reached, where the addition of more potential active sites does not result in a meaningful increase in $\mathrm{O}_{2}$ production. This means that only a fraction of the film is participating in the reaction, though the exact percentage is difficult to estimate.

A hypothesis, and accompanying physical description, that explains the data from this chapter and that of Chapter II is as follows. First, the higher the deposition current density, the more dense the layers deposited. This leads to better charge transport through the film, at the expense of mass transport. These films are thus limited mainly by mass transport, with any improvements in charge transport gained from additional pulses (increasing film connectivity) generally outweighed by further losses in mass transport. The more porous nature of the films deposited under low current density conditions behave differently. They are able to realize the gains in charge transport from additional pulses without the significant losses from mass transport, meaning they maintain sufficient porosity.

One interesting area for further exploration is to expand the pulse duration of some of these high deposition current densities. The data from our earlier work superimposed on Figure 4.2 shows that films deposited at lower current densities (-10 $\mathrm{mA} \mathrm{cm}{ }^{-2}$ ) with longer pulse durations $(2 \mathrm{~s})$ show continued improvements in TOF with loading well beyond where those of the shorter duration, higher deposition current densities began to diminish. This can be explained by thinking about how film growth changes with time. 
Initially, films may deposited in a fairly organized (high packing density) way. As the deposition continues, and the film grows, new material is precipitated randomly on the surface. The longer the deposition is allowed to continue, the greater the inefficiency with which the material is loaded, leading to a more porous film. For films deposited under low current densities that are limited by charge transport, this inefficiency is unwanted, as it exacerbates the problem, as shown by a decrease in TOF with loading for continuously deposited films. For films deposited under higher current densities however, that are limited by mass transport, intentionally expanding the duration of the deposition pulses, and in turn increasing the porosity, may actually extend the maximum performance of the film to higher loadings.

\section{Conclusions and Bridge}

In conclusion, we have shown that film performance is governed by a goldilocks principle, where mass and charge transport determine the ideal loading. At low overpotentials, high current density depositions provide better performance when normalized to the amount of material deposited. At high overpotentials, the opposite is true, as the high deposition current density films experience a loss in bulk activity due to mass transport limitations, though the total (unnormalized) current passed at equal loadings is still greater.

Having demonstrated ways to optimize the performance of films at high loadings, such as would be suitable for industrial electrolyzers, we turn now to the opposite end of the catalyst loading spectrum. In Chapter V, we discuss how the unique requirement of 
optical transparency in photoelectrochemical cells imposes new demands, with device performance now a function of both the optical and catalytic properties of a film. 


\title{
CHAPTER V
}

\section{IN SITU CHARACTERIZATION OF THE OPTICAL PROPERTIES OF ELECTROCATALYSTS}

\author{
Portions of this chapter were previously published as Batchellor, A.; Trotochaud, \\ L.; Boettcher, S. W. In Situ Characterization of the Optical Properties of Electrocatalysts, \\ In Advanced and In Situ Analytical Methods for Solar Fuel Materials 2015, Volume 371 \\ of the series Topics in Current Chemistry, pp 253-324. A.S.B. wrote the manuscript. \\ L.T., having performed much of the cited experimental work, provided insights and \\ clarifications. S.W.B. was the principal investigator and provided editorial assistance.
}

\section{Introduction}

The development of efficient photoelectrochemical (PEC) water-splitting cells necessitates the use of electrocatalysts at the anode and cathode surfaces to help reduce the overpotentials associated with the oxygen and hydrogen evolution half-reactions. To avoid using spatially separate light absorbing (photovoltaic) and water splitting (electrolyzer) devices to drive the evolution of $\mathrm{O}_{2}$ and $\mathrm{H}_{2}$, catalysts can be deposited directly onto the light absorbing semiconductor electrodes (or buried photovoltaic junction). One major challenge that arises from physically coupling the catalysts and photoelectrodes in this way is that catalysts parasitically absorb light when operated 
under the electrochemical conditions required for water splitting. ${ }^{1}$ This parasitic absorption by the catalyst reduces the number of photons that are able to reach the electrode surface, and thereby limits the number of electron-hole pairs that can be created and used to drive the redox reactions.

Often, the efficiency of the individual components (e.g. catalysts, semiconductor electrodes) that would complete a water-splitting cell is investigated. ${ }^{2,3}$ Recently, an increase in both modelling and experimental work is being done to see how these components fair in composite systems. ${ }^{4-21}$ While high-throughput screening has been used to predict which catalysts may contain ideal optical properties (low absorption), ${ }^{22}$ little work has directly investigated the photocatalytic properties of the current state-ofthe-art catalysts. In situ ultraviolet/visible (UV-Vis) spectroscopy is a valuable method for quantitatively evaluating the optical properties of catalysts under working conditions. The light absorbing effects can then be incorporated into "opto-electrochemical" models of composite water-splitting cells.

\section{Electrochromism in Catalyst Materials}

Electrochromism is the process through which a material changes color in conjunction with a redox reaction. As many catalysts are (semi)transparent upon deposition, and become darker upon oxidation, a general description of their electrochromic behavior is as follows:

$$
\underset{\text { bleached }}{\mathrm{R}} \rightarrow \underset{\text { colored }}{\mathrm{O}}+\mathrm{n} e^{-}
$$


This behavior can be valuable for applications including "smart" windows capable of improving the energy efficiency of buildings. ${ }^{23,24}$ However, the same behavior can negatively affect the performance of composite catalyst/semiconductor water-splitting photoelectrodes where the incident photons must first pass through the (colored) catalyst prior to being productively absorbed in the semiconductor.

Seike and Nagai used in situ spectroscopy to investigate the electrochromic properties of $3 \mathrm{~d}$ transition metal oxides (TMOs) (e.g. $\left.\mathrm{NiO}_{\mathrm{x}}, \mathrm{CoO}_{\mathrm{x}}, \mathrm{TiO}_{\mathrm{x}}, \mathrm{MnO}_{\mathrm{x}}, \mathrm{CrO}_{\mathrm{x}}\right){ }^{25}$ These materials are important in the context of solar fuels because many of the same $3 \mathrm{~d}$ TMOs have drawn attention as water oxidation catalysts due to their relatively large abundance and low cost compared to precious metal catalysts. In the colored, highoptical-absorption state, electrochromic $\mathrm{TMOs}$ such as $\mathrm{NiO}_{\mathrm{x}}$ would parasitically absorb photons en route to the semiconductor surface, thus diminishing PEC performance. Seike and Nagai found that $\mathrm{NiO}_{\mathrm{x}}$ has a high coloration efficiency compared to the other $3 \mathrm{~d}$ TMOs studied. Practically, this means that very little charge needs to be injected (via oxidation of the film) to cause a large change in the optical density of $\mathrm{NiO}_{\mathrm{x}}$. The source of this coloration process is not well understood, despite considerable efforts to understand the electronic properties of the material. Possible sources have been postulated to include crystal defects ${ }^{26}$, the presence of high valency $\mathrm{Ni}$ (to include $\left.\mathrm{Ni}^{4+}\right)^{27}$, [metal oxygen charge transfer ${ }^{28}$, and phase changes arising due to ion insertion ${ }^{29}$.

$\mathrm{NiO}_{\mathrm{x}}$, which converts to $\mathrm{Ni}(\mathrm{OH})_{2}$ following electrochemical conditioning in basic media ${ }^{30}$, is one of the most promising OER catalysts when small concentrations $(\sim 10 \%)$ of Fe are added..$^{31}$ Under conditions relevant to the OER in base, the reaction is

$$
\underset{\text { transparent (light green) }}{\mathrm{Ni}(\mathrm{OH})_{2}}+\underset{\text { dark colored }}{\mathrm{OH}^{-}}+\mathrm{H}_{2} \mathrm{O}+\mathrm{e}^{-}
$$


To maintain charge neutrality, the transfer of electrons between the catalyst and the electrode must be accompanied by the transfer of ions into or out of the film. Under OER conditions, every Ni site exposed to electrolyte, whether at the surface of the $\mathrm{Ni}(\mathrm{OH})_{2}$ or as part of a disordered (i.e. only locally ordered), ion-permeable (oxy)hydroxide phase, will be oxidized and thus contribute to optical absorption.

\section{In Situ UV-Vis Absorption and Reflection Spectroscopy}

From the above discussion of electrochromism, it is apparent that the optical properties of OER catalysts must be measured in situ to understand and account for their effects on photoabsorption in the underlying semiconductor elements in a photoelectrochemical cell. A spectrophotometer can measure the amount of light that passes through the catalyst, which is the optical transmittance. Example in situ experimental setups employed by Trotochaud et al. and Corrigan and Knight are shown in Figure 5.1. ${ }^{1,27}$ 
a

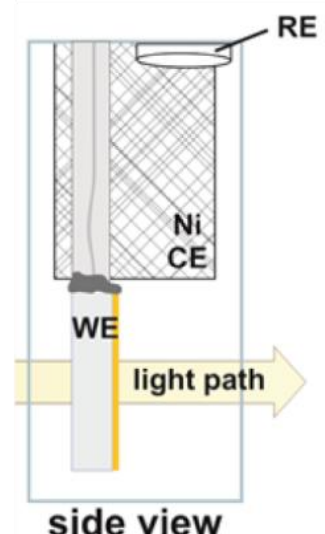

side view b

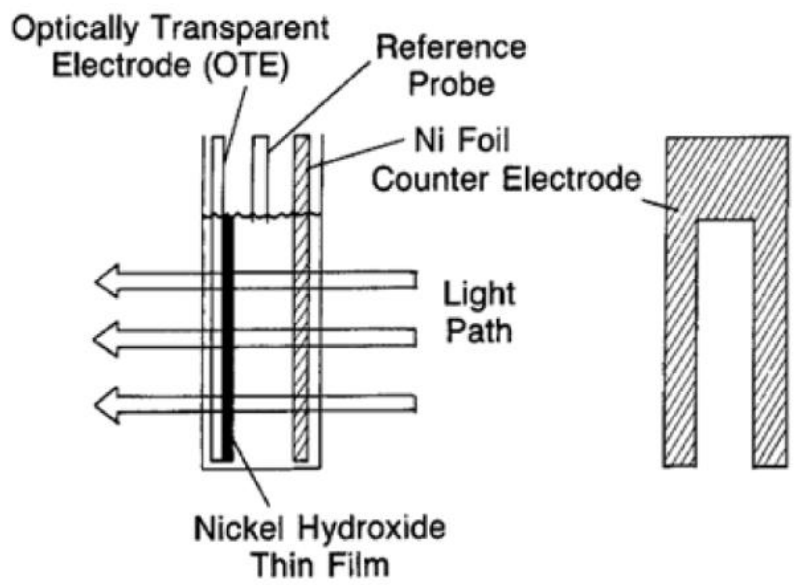

Figure 5.1. In situ electrochemical cells employed by a) Trotochaud et al. ${ }^{1}$ and b) Corrigan and $\mathrm{Knight}^{27}$ to collect optical spectroscopy data on catalysts films. In A), thinfilm electrocatalysts were deposited by spin-coating onto the ITO substrate prior to electrode fabrication.

In these cells, the electrochemical potential of the catalyst can be controlled and poised at/near the potential of OER onset while the optical spectra are recorded. The catalysts are typically supported on a transparent conducting oxide electrode. In Figure 5.1a the counter electrode (CE) was held above the path of the incident light and in Figure 5.1b the CE had a section removed to permit the incident light to pass through. We note that in this setup there can be a significant (diffuse) scattered component to transmission and reflection spectra (especially for thicker films), therefore these measurements are ideally made in an integrating sphere. In the work by Trotochaud et al., a second cell was designed for use with an integrating sphere to collect reflectance spectra and very thin films were used for the in situ transmission measurements where diffuse scattering was negligible.

The transmittance of a $\mathrm{NiOOH} / \mathrm{Ni}(\mathrm{OH})_{2}$ catalyst over a range of potentials is shown in Figure 5.2. The transmittance was nearly independent of potential from 0 to 
$0.45 \mathrm{~V}$ vs. $\mathrm{Hg} / \mathrm{HgO}$, where the catalyst remains in its bleached state and $\mathrm{Ni}$ is formally in the $2+$ oxidation state. Then, as the $\mathrm{Ni}$ in the film was oxidized to $3^{+} / 4^{+}$over the potential range from 0.45 to $0.55 \mathrm{~V}$ vs. $\mathrm{Hg} / \mathrm{HgO}$, the catalyst became colored, and the transmittance decreased significantly. Once colored (i.e. at potentials higher than the Ni oxidation wave), the transmittance showed little variation as the potential was further increased.
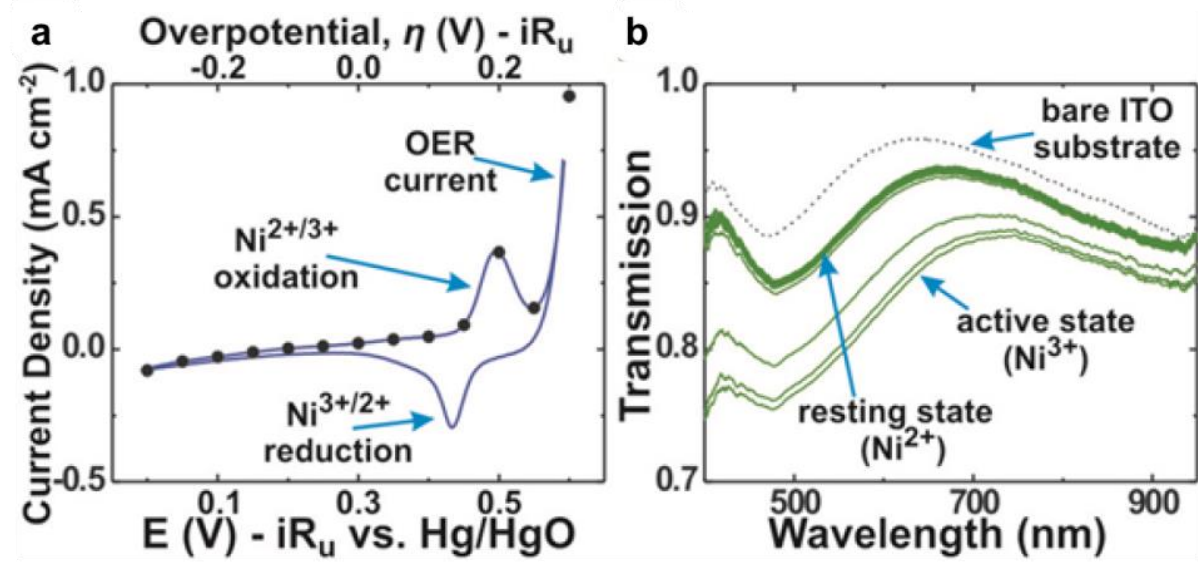

Figure 5.2. a) Cyclic voltammetry (CV) and b) transmission spectra of $\mathrm{NiO}_{\mathrm{x}}$ film measured at potentials ranging from 0 to $0.6 \mathrm{~V}$ vs $\mathrm{Hg} / \mathrm{HgO}$ in $0.05 \mathrm{~V}$ increments. Each line in $\mathrm{b}$ ) was taken at a potential corresponding to the dots on the $\mathrm{CV}$, with the top transmission line corresponding to the lowest potential. Adapted from. ${ }^{1}$

All incident photons on a surface must be absorbed, reflected or transmitted:

$$
1=A+R+T
$$

where $A, R$, and $T$ are the wavelength-dependent absorption, reflection and transmission processes, respectively. The fraction of light passing through a material $I$ with respect to the incident (non-reflected) light $I_{\mathrm{o}}$ follows Beer's law as given by:

$$
I=I_{\mathrm{o}} \mathrm{e}^{-\alpha t}
$$


where $\alpha$ is the absorption coefficient, which is an intrinsic property of the solid, and $t$ is the thickness. The transmittance of a material is related to $\alpha$ by:

$$
T=\left(I / I_{\mathrm{o}}\right)=\mathrm{e}^{-\alpha t}
$$

If $\alpha$ is known for a given material, the optimal thickness $t$ of catalyst film for use with a photoelectrode can be determined such that $T$ is maximized over the wavelength region of interest for the photoelectrode while maintaining a low overpotential for the reaction of interest. However, directly determining $\alpha$ for a catalyst in situ can be challenging. This is because for any particular measurement, the measured value of $T$ is that for the entire electrochemical/optical cell, not simply the transmittance of the catalyst layer $T_{c}$. The optical effects of the cell walls (typically glass or quartz) as well as the underlying transparent conductive oxide (TCO) substrate need to be properly accounted for such that the intrinsic wavelength-dependent absorption by the catalyst film $a_{c}(\lambda)$ can be extracted. This is important if the optical data collected from catalyst films on TCO electrodes is to be used to predict optical effects of the catalyst on different semiconductor photoelectrodes. To accomplish this, an optical model is needed that accounts for the reflection $(r)$ and absorption $(a)$ probabilities at each of the various interfaces the light encounters during the experimental measurement. These events are depicted in Figure 5.3. Approximations to such a model (for instance, ignoring various higher order reflection terms) to make its solution analytically tractable are discussed in Trotochaud et al. ${ }^{1}$ 


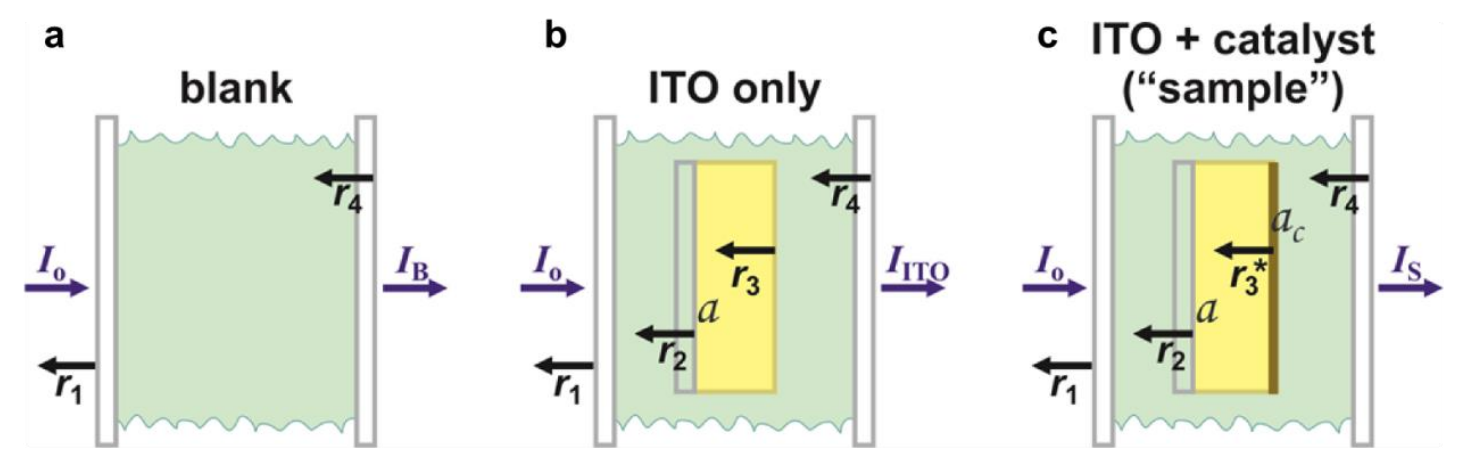

Figure 5.3. Cross-sectional view showing the light path through an in situ liquid cell. The symbols $a$ and $r$ signify absorption and reflection probabilities, respectively, with the subscripts indicating the location of the optical event as described below. The spatial location of the arrows and symbols along the vertical scale of the diagrams is not significant. Adapted from. ${ }^{1}$

From $a_{\mathrm{c}}(\lambda)$, the transmission probability at a given wavelength once the photon has reached the catalyst, $T_{\mathrm{c}}(\lambda)$, can then be found by:

$$
T_{\mathrm{c}}(\lambda)=1-a_{\mathrm{c}}(\lambda)
$$

and the absorption coefficient of the catalyst $\alpha(\lambda)$ can be calculated from:

$$
\alpha(\lambda)=-\ln T_{\mathrm{c}}(\lambda) / t
$$

where $t$ is the thickness of the film. Figure 5.4 shows the extracted absorption coefficients of the $\sim 2 \mathrm{~nm}$ thick transition-metal oxide water oxidation catalysts studied by Trotochaud et al., as well as the calculated transmission probabilities for a mixed Ni/Fe oxyhydroxide film as a function of film thickness. The transmission losses are significant even for films only a few nanometers thick. 
a

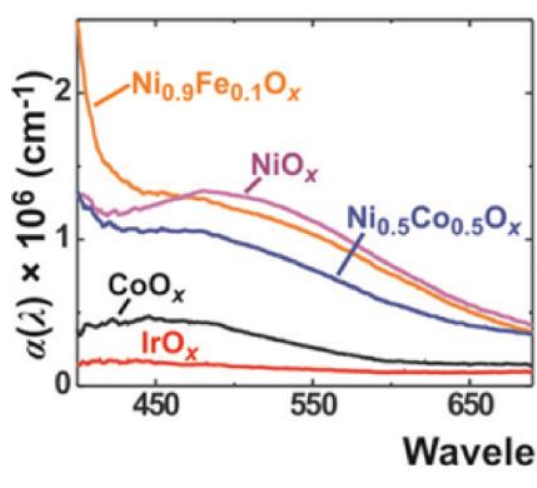

b

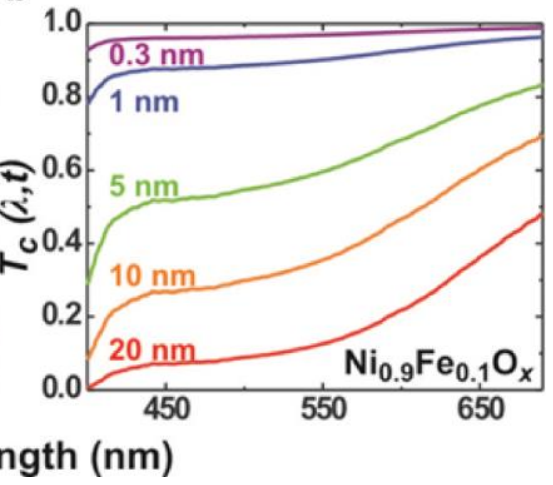

Figure 5.4. a) Effective absorption coefficient $\alpha(\lambda)$ calculated for the active catalyst films. b) Transmission probabilities $T_{c}(\lambda)$ calculated for $\mathrm{Ni}_{0.9} \mathrm{Fe}_{0.1} \mathrm{O}_{x}$ films of varying thickness. Adapted from. ${ }^{1}$

For catalysts, this thickness dependence of the transmission probability poses an interesting problem, as two competing effects are pitted against one another; increasing the catalyst loading lowers the overpotential required to pass a given oxygen-evolution current, reducing voltage losses from kinetics. However, a thicker catalyst layer will also parasitically absorb more light, thus decreasing the total photocurrent generated by the photoanode. Furthermore, the high coloration efficiency of Ni discussed previously manifests itself here, as the values of $\alpha(\lambda)$ measured in situ are seen to be greater for the Ni-based catalysts than those of the other metal oxides studied.

\section{Identification and optimization of catalyst films for solar water splitting}

\section{photoanodes}

The ultimate measure of an electrocatalyst's utility for photoelectrochemical water splitting is how it performs in a prototype device. Defining reasonable figures-ofmerit allow one to determine which catalysts offer the best combination of high turnover 
frequency (mol product / mol active site / s) at low overpotential and low parasitic optical absorption, without the need to individually test each particular catalyst in a complete solar water splitting device as a function of catalyst loading. Trotochaud et al. and Gregoire et al. described figures of merit based on experimentally measured data. ${ }^{1,22}$

The approach described by Trotochaud et al. is summarized here, and later compared to that of Gregoire et al. The amount of light at a given wavelength $\lambda$ reaching the semiconductor surface is a function of the incoming light flux, $F(\lambda)$, multiplied by the corresponding catalyst transmission probability, $\mathrm{T}_{\mathrm{c}}(\lambda, t)$. The optical efficiency, $\varphi_{\mathrm{opt}}$, is defined as

$$
\varphi_{\mathrm{opt}}(t)=\int \mathrm{F}(\lambda) \cdot \mathrm{T}_{\mathrm{c}}(\lambda, t) / \int \mathrm{F}(\lambda)
$$

over the range of the electromagnetic spectrum available to the photoelectrode as determined by its band gap (for example, a n- $\mathrm{Fe}_{2} \mathrm{O}_{3}$ photoanode with a band gap of 2.2 $\mathrm{eV}$ will only absorb photons with $\lambda \leq(1240 / 2.2) \approx 560 \mathrm{~nm})$. The optical efficiency is a function of the catalyst film thickness (i.e. catalyst loading) due to the dependence of $T_{c}$ on $t$, as shown in Figure 5.5.

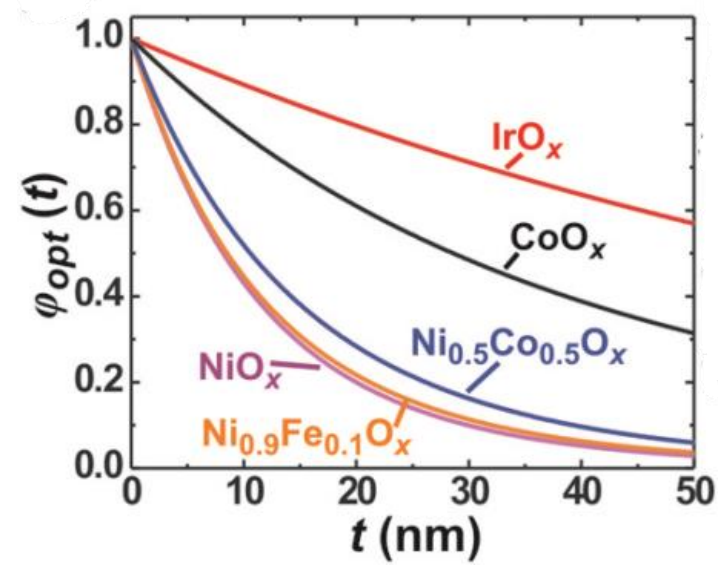

Figure 5.5. Optical efficiency $\varphi_{\mathrm{opt}}(t)$ of active catalyst films as a function of thickness $t$, assuming an optical band gap $1.8 \mathrm{eV}$ for the absorbing semiconductor and AM 1.5 solar flux. Adapted from. ${ }^{1}$ 
For thin films $<1 \mathrm{~nm}$, the $\varphi_{\text {opt }}(t)$ of all catalysts approaches unity, as there is too little material to absorb a significant fraction of incoming photons. For films $>50-100$ $\mathrm{nm}$ in thickness, $\varphi_{\mathrm{opt}}(t)$ trends towards zero - even relatively transparent films will absorb the majority of photons as their thickness becomes sufficiently large. It is interesting to note that due to the high absorption coefficient of Ni-based catalyst films, their corresponding $\varphi_{\mathrm{opt}}(t)$ values are much lower than those of the Ni-free films.

Combining the optical properties with the independently measured catalytic properties (we assume the activity, i.e. catalytic current density at a given overpotential, scales linearly with film thickness) ${ }^{30}$ allows us to define the optocatalytic efficiency of the films, $\Phi_{\mathrm{O}-\mathrm{C}}$.

$$
\Phi_{\mathrm{O}-\mathrm{C}}=\mathrm{P}_{\max } / \mathrm{P}_{\mathrm{o}, \max }
$$

$\mathrm{P}_{\mathrm{o}, \max }$ is the maximum power achievable in the electrode with a hypothetical "perfect" catalyst that has no parasitic optical absorption and infinitely fast kinetics for $\mathrm{O}_{2}$ evolution, and $\mathrm{P}_{\max }$ is the power achievable with the addition of the real catalyst (including optical and kinetic voltage losses). Trotochaud et al. calculated power curves and then determined $\Phi_{\mathrm{O}-\mathrm{C}}$ values for some of the most promising catalysts as seen in Figure 5.6. 
a

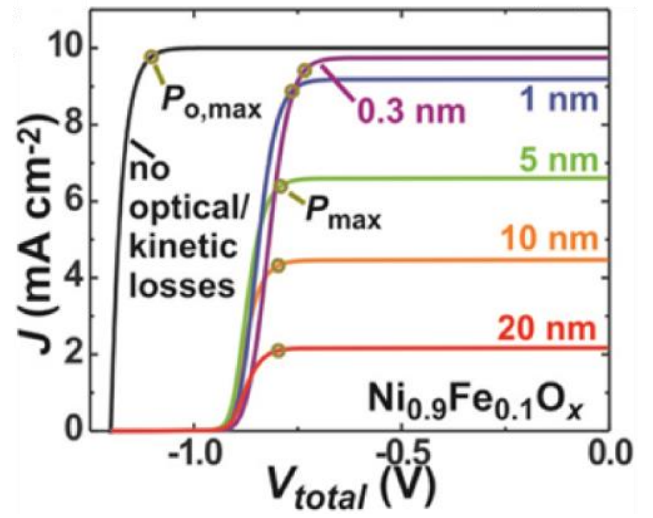

b

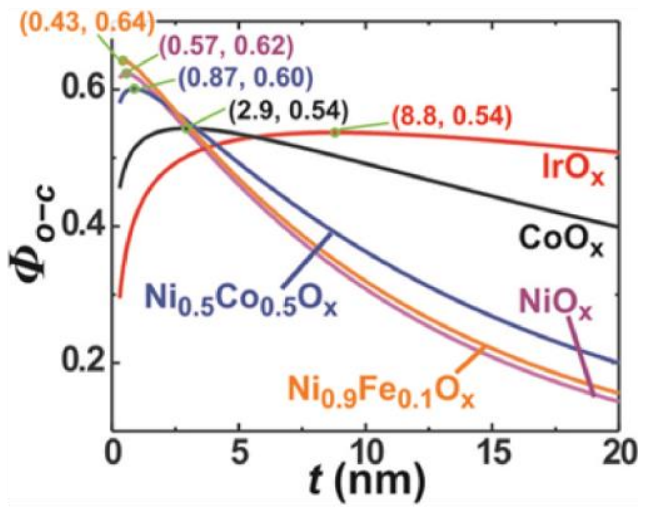

Figure 5.6. a) Photoelectrode current voltage curves for $\mathrm{Ni}_{0.9} \mathrm{Fe}_{0.1} \mathrm{O}_{\mathrm{x}}$ and b) Optocatalytic efficiency $\Phi_{\mathrm{O}-\mathrm{C}}$ as a function of thickness $t$ for different catalysts, assuming an optical band gap $1.8 \mathrm{eV}$ for the absorbing semiconductor and AM 1.5 solar flux. Adapted from. ${ }^{1}$

All catalyst films studied reached their maximum $\Phi_{\mathrm{O}-\mathrm{C}}$ at less than $10 \mathrm{~nm}$. The highest performing system is predicted to be the $\mathrm{Ni} / \mathrm{Fe}$ catalyst with a $\Phi_{\mathrm{O}-\mathrm{C}}$ value of 0.64 . This $\Phi_{\mathrm{O}-\mathrm{C}}$ is achieved with a film thickness of less than $0.5 \mathrm{~nm}$. In fact, all Ni-based catalysts had their max $\Phi_{\mathrm{O}-\mathrm{C}}$ values at less than $1 \mathrm{~nm}$ thickness, with a steep drop-off in efficiency with increasing thickness. This is due to their high absorption coefficient as mentioned previously. $\mathrm{IrO}_{\mathrm{x}}$, on the other hand, is predicted to reach its maximum $\Phi_{\mathrm{O}-\mathrm{C}}$ at $8.8 \mathrm{~nm}$, due to its lower absorption coefficient. In the case of $\mathrm{IrO}_{\mathrm{x}}$, the advantage of more-transparent catalysts in the trade-off between lower overpotential and higher parasitic absorption is exemplified; an incremental increase in the thickness of $\mathrm{IrO}_{\mathrm{x}}$ up to $10 \mathrm{~nm}$ decreases the voltage loss more substantially than it increases the parasitic light absorption.

In work by Gregoire et al., a related optocatalytic FOM is proposed. ${ }^{22}$ In order to determine the optocatalytic efficiencies of over 5000 catalysts via high-throughput experimentation, only a snapshot of the catalysts' properties at $0.45 \mathrm{~V}$ overpotential were input into the model. Also the thickness-dependence was not investigated (which would 
have been challenging in such a high-throughput screening approach) and thus no ideal parameters were determined for any one particular catalyst. The optical properties were also not screened in situ and therefore any electrochromic behavior was not accounted for. Despite the different goals and approaches, the two works come to some similar conclusions, namely that a Ni/Fe-based film would be a good catalyst for a composite PEC device.

\section{Tailoring of Catalyst Optical Properties}

When the relevant properties of a catalyst (e.g. $a_{c}$ ) have been ascertained, it may be possible to tune the optical properties of the catalyst by adjusting composition while not affecting the catalyst behavior. In effort to develop "optically passive" counter electrodes for electrochromic applications, Azens et al. have shown that by varying the Ce concentration in Ti and $\mathrm{W}$ films, they were able to create a transparent film without the typically observed electrochromic response. ${ }^{32}$ In a related study, Monk et al. show that by mixing various TMOs in amorphous multi-cation oxides the wavelength of the maximum in the electrochromic absorption band can be tailored. ${ }^{33}$ Extending these approaches to water oxidation catalysts, it could be possible to adjust the optical properties of a film without a significant change in catalytic performance. Given the large range of compositions including $\mathrm{Ni}, \mathrm{Fe}, \mathrm{Ce}, \mathrm{Ti}$, Co etc. now found to have relatively

high catalytic activity ${ }^{3,22,34,35}$ there are likely mixed oxide compositions that could be found which also have low optical absorption. 
For example, Gregoire et al. found two regions of interest in the quaternary system studied. ${ }^{22}$ The first is the high catalytic performance of the predominately Ni/Fe system, while the other is the high optocatalytic performance with a film composition of $\mathrm{Fe}_{0.23} \mathrm{Co}_{0.13} \mathrm{Ni}_{0.07} \mathrm{Ti}_{0.57} \mathrm{O}_{\mathrm{x}}$. This particular system is interesting because of the (relatively) poor catalytic performance of films containing significant concentrations of Ti. However, the extremely high transmission properties of films containing large concentrations of $\mathrm{Ti}$ are capable of overcoming its catalytic limitations with respect to the combined figure of merit. It is reasonable to assume that a system with a large Ti content would likely never have been explored by researchers searching solely for a highly catalytically active catalyst. This identification opens up a new system to be explored and optimized and exemplifies the value of high throughput investigations.

\section{New Horizons for In Situ Optical Spectroscopy for Solar Fuels Material Characterization}

Beyond determining the optical properties of catalyst films in the context of solar fuels, there are other directions that can be taken to exploit the benefits of in situ optical spectroscopy. Due to the electronic properties of certain elements, analytical techniques are sometimes incapable of acquiring the same data on all materials of interest. Brückner used in situ parallel UV-Vis/EPR/on-line gas chromatography to investigate the oxidation state of a chromium oxide catalyst during the dehydrogenation of propane ${ }^{36} \mathrm{Cr}^{6+}$ is not detectable by EPR, but EPR is able to distinguish between $\mathrm{Cr}^{3+}$ and $\mathrm{Cr}^{5+} \cdot \mathrm{Cr}^{6+}$ is identifiable via UV-Vis spectroscopy through its charge-transfer transitions. By 
measuring the UV-Vis spectra as the temperature was varied, the absorption band was shown to disappear as relevant reaction temperatures were reached, ruling out $\mathrm{Cr}^{6+}$ as a possible active site. In a separate work, Brückner used in situ parallel UVVis/EPR/Raman spectroscopy to elucidate the oxidation state of $\mathrm{V}$ in a $\mathrm{V} / \mathrm{TiO}_{2}$ film ${ }^{37}$, showing that several species of $\mathrm{V}^{4+}$ are actively engaged in the dehydrogenation of propane.

In composite PEC cells, the catalyst films are not the only material that will interfere with the incoming flux of light. Due to the production of $\mathrm{H}_{2}$ and $\mathrm{O}_{2}$ at the electrode surfaces, the presence of bubbles will also need to be accounted for. The decrease in efficiency caused by the formation of bubbles will come from both ohmic $\operatorname{losses}^{38}$ as well as optical losses due to enhanced scattering ${ }^{39-43}$. As the bubbles are only formed under reaction conditions, the use of in situ optical techniques will be paramount to the quantification of these losses.

\section{Summary}

In conclusion, if catalysts are to be utilized in a composite water splitting cell, both their catalytic and optical properties need to be optimized. In situ optical methods such as UV-Vis spectroscopy provide the basis for combined optocatalytic figures of merit and guide the design of improved materials through tailoring of the catalyst absorption bands and electrochromic properties as well as potentially providing insight into catalyst mechanisms. 


\section{CHAPTER VI}

\section{SUMMARY AND OUTLOOK}

\section{Summary}

The research discussed in this dissertation addresses important knowledge gaps in the field of OER catalysts. In Chapter II, we discussed best practices for measuring and reporting intrinsic activities, highlighting experimental conditions where the film activity on a per-metal-cation basis can be accurately measured and where capacitance measurements of electrochemically active surface area fail. We found that the

electrochemical reversibility of the (nominally) $\mathrm{Ni}^{2+/ 3+}$ redox couple correlates with the apparent intrinsic activity as a function of loading. We reported a pulsed-

electrodeposition method that dramatically improves the catalyst reversibility and performance at high loading compared to continuous electrodeposition, which we attribute to improved connectivity in the micro/nanostructure and better composition control.

In Chapter III, we reported the effects of electrodeposition technique (pulsed versus continuous), electrochemical cycling, and Fe content on the structure of the $\mathrm{Ni}_{(1-}$ z) $\mathrm{Fe}_{z} \mathrm{O}_{x} \mathrm{H}_{y}$ catalyst film as determined using PDF analysis. The PDF patterns for the films are best simulated by model structures consisting of brucite-like $\beta-\mathrm{Ni}(\mathrm{OH})_{2}$ fragments 1 to 3 layers in thickness. Only the oxidation state of the film was found to significantly affect the intra-layer scattering behavior (i.e. metal-oxygen bond distance). The interlayer interactions, however, were shown to be affected by Fe content and deposition 
conditions. We found that films can be cycled between as-deposited, oxidized, and reduced states, with minimal loss of intrasheet coherence, indicating a degree of structural stability.

In Chapter IV, a design of experiments analysis demonstrated that the deposition current density played a larger role than both the duration of the deposition and the duration of stirring between electrochemical pulses. Higher deposition current densities than were traditionally used by this lab were explored, and a greater than two-fold improvement in activity at high loading was obtained compared to previous work in the group. The identification of an ideal loading range, explained through the competition between mass and charge transport, is an important step towards understanding the scalability of these films towards industrial conditions.

In Chapter V, a broad treatment of the current work in the photoelectrochemical properties of thin film OER catalysts was provided. The unique requirement of optically transparent catalysts in a combined photovoltaic-electrolyzer cell requires the use of combined optical and catalytic figures of merit. This requirement of optical transparency necessitates the use of thin films for many non-transparent, but catalytically active, materials, and mitigates the effects of charge and mass transport that dominated the high loading films of Chapter II. As the optical properties of many catalysts change with potential, in situ techniques are essential for their measurement. Furthermore, in situ optical measurements are shown to be useful in probing the oxidation state of the active catalyst material, which could lead to better understanding of the underlying OER mechanism.

\section{Outlook}




\section{Benchmarking}

The most obvious need for the field of OER catalysis moving forward is the adoption of a series of benchmarking parameters. While there is general agreement on certain catalysts being high performing (i.e., $\mathrm{NiFeO}_{\mathrm{x}} \mathrm{H}_{\mathrm{y}}$ ), there are as of now no objective parameters by which to make that judgement. A first step requires that all activity metrics reported must be normalized to the amount of material participating in the reaction. This is due to the presence, perhaps even prominence, of volume-active catalysts. There is notably an amount of ambiguity in whether this should constitute the entirety of the material deposited or simply the electrochemically active surface area. While the latter may be more fundamentally valuable, as it provides a better estimate of the intrinsic activity of the active species, the former provides a more level playing field, as the use of inert supports is common, and all materials will be subject to the cost and volume constraints of devices.

The identification of a true intrinsic activity is complicated by several factors highlighted in Figure 6.1. 


\section{Complications Towards Measuring an Intrinsic TOF}

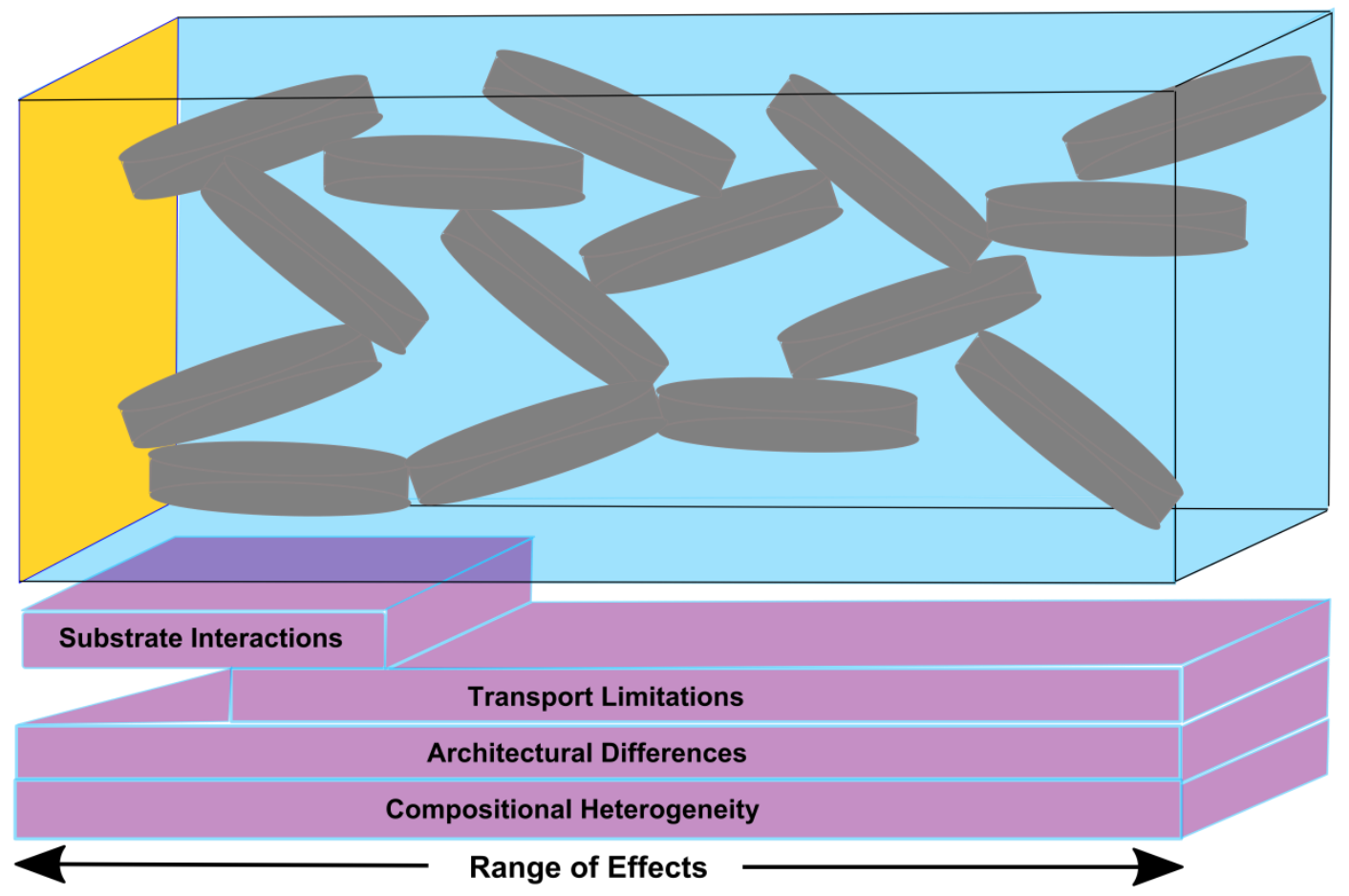

Figure 6.1. Cartoon highlighting factors that complicate the measurement of a true intrinsic TOF and their relative effective range.

Near the electrode surface, substrate effects may alter the performance of the film if the film is attached through some form of chemical bonding instead of merely a physical adsorption. Transport limitations serve to mask the true activity of the film by the creation of potential and concentration gradients across the film. Architectural differences arise depending on the deposition method and electrochemical conditioning protocol. Compositional heterogeneity may arise due to the preferential deposition of one species from a mixed solution, or from phase segregation due to low solid phase solubility of a dopant. Elimination of any, let alone all, of these effects is non-trivial, and great care should be taken to understand, and report, to what degree these affect the measured average activity. 
An invaluable benchmark upon which groups could generate activity comparisons would be through the use of a standard film, prepared from the same materials, and tested under identical conditions, generating data to support field-specific figures of merit. This would allow for intergroup transparency, and claims of high activity would thus have to beat a universally accepted threshold. The standard set of experiments and tools employed should aid in quantification, both pre- and post-electrochemistry, of material stoichiometry and morphology, which are essential for truly understanding the role of the various components of the film. This is especially relevant for materials claiming to stabilize metallic species that should be soluble at the operating $\mathrm{pH}$. There is also a need for reporting any decline in activity with time, and if possible, the source of the transient behavior.

\section{The Need for In Situ Characterization}

Because OER happens at anodic potentials, and generally proceeds following the oxidation of one of the transition metals in the film, acquisition of resting-state information is inherently limiting. A true picture of the optical and structural properties of the active material can only be obtained when the oxidized structure is present, which due to self-discharge, may not be obtainable in the absence of an applied potential. The design of cells that can facilitate both electrochemistry and data collection is challenging due to the presence of the electrolyte solution. Under ambient conditions, this requires deposition of the catalyst on extremely thin, transparent electrodes. Under (near) vacuum conditions, the additional requirement of a fully-enclosed flow cell further complicates matters. While some in situ cell setups exist for general use at various beamlines, the 
publishing of "blue prints" for field specific cells is rather limited, especially in contrast to analytical software which is readily distributed. This severely hurts the data acquisition capabilities of new and underfunded groups, and hinders progress in the field.

\section{Beyond Pourbaix}

Another area of need is to further explore the limitations of material stability as reported in Pourbaix diagrams. While these predictors are likely reliable for the predominant component of an OER material, they do not intend to describe the thermodynamics (certainly not the kinetics) of the phase transitions of minor components supported in a stable film, and thus extrapolating the phase of a minor component from its own monometallic diagram should be done with caution, if at all. This could open up a range of dopant materials previously ignored due to their presumed solubility in alkaline or acidic solutions.

\section{Deposition Mechanisms and Film Architectures}

While there still exists the possibility for discovering materials with activity higher that of $\mathrm{NiFe}$ oxyhydroxides, the opportunity to construct an ideal film architecture through optimization of deposition conditions has received far less attention. To achieve this, the underlying mechanisms by which films are deposited must be understood. The transient activity and structure of films is often reported, but the potential for transient behavior during deposition may be equally important, as highlighted in Chapter II, especially in slow, low temperature syntheses where local environments deviate from the bulk and kinetic products are more likely. 
While many groups have attempted to deposit high performing catalysts on inert architectures to improve either the surface area or conductivity, this ignores the rich variability in film morphologies that can be achieved. No true roadmap yet exits for synthesizing a "model" transition metal film. Though fundamental insights can be gained through the use of sacrificial templates or other advanced synthesis methods, a basic understanding of the deposition specific morphologies of both rigorously pure and codeposited metals is needed.

\section{Becoming True Material Scientists and Engineers}

Finally, it is imperative that we expand the breadth of our knowledge in order to keep abreast of the insights obtained in adjacent fields (i.e., batteries and capacitors). The pioneering work of Dennis Corrigan highlighting the importance of Fe in catalyzing water oxidation, a reaction he hoped to suppress in batteries, is a prime example. Further insights, overlooked, are likely out there if we are willing to hold ourselves up as true material scientists, and not simply practitioners of electrocatalysis. 


\section{REFERENCES CITED}

\section{Chapter I}

(1) Lewis, N. S.; Nocera, D. G. Powering the Planet: Chemical Challenges in Solar Energy Utilization. Proc. Natl. Acad. Sci. U. S. A. 2006, 103, 15729-15735.

(2) Pinaud, B. a.; Benck, J. D.; Seitz, L. C.; Forman, A. J.; Chen, Z.; Deutsch, T. G.; James, B. D.; Baum, K. N.; Baum, G. N.; Ardo, S.; et al. Technical and Economic Feasibility of Centralized Facilities for Solar Hydrogen Production via Photocatalysis and Photoelectrochemistry. Energy Environ. Sci. 2013, 6, 1983.

(3) Walter, M. G.; Warren, E. L.; McKone, J. R.; Boettcher, S. W.; Mi, Q.; Santori, E. A.; Lewis, N. S. Solar Water Splitting Cells. Chem. Rev. 2010, 110, 6446-6473.

(4) Kim, D.; Sakimoto, K. K.; Hong, D.; Yang, P. Artificial Photosynthesis for Sustainable Fuel and Chemical Production. Angew. Chemie Int. Ed. 2015, 54, 3259-3266.

(5) Cook, T. R.; Dogutan, D. K.; Reece, S. Y.; Surendranath, Y.; Teets, T. S.; Nocera, D. G. Solar Energy Supply and Storage for the Legacy and Nonlegacy Worlds. Chem. Rev. 2010, 110, 6474-6502.

(6) Yeo, B. S.; Klaus, S. L.; Ross, P. N.; Mathies, R. a; Bell, A. T. Identification of Hydroperoxy Species as Reaction Intermediates in the Electrochemical Evolution of Oxygen on Gold. Chemphyschem 2010, 11, 1854-1857.

(7) Hall, D. E. Alkaline Water Electrolysis Anode Materials. J. Electrochem. Soc. 1985, 132, 41C-48C.

(8) Marini, S.; Salvi, P.; Nelli, P.; Pesenti, R.; Villa, M.; Berrettoni, M.; Zangari, G.; Kiros, Y. Advanced Alkaline Water Electrolysis. Electrochim. Acta 2012, 82, 384391.

(9) Zeng, K.; Zhang, D. Recent Progress in Alkaline Water Electrolysis for Hydrogen Production and Applications. Prog. Energy Combust. Sci. 2010, 36, 307-326.

(10) Kang, D.; Kim, T. W.; Kubota, S. R.; Cardiel, A. C.; Cha, H. G.; Choi, K.-S. Electrochemical Synthesis of Photoelectrodes and Catalysts for Use in Solar Water Splitting. Chem. Rev. 2015, 115, 12839-12887.

(11) Navarro Yerga, R. M.; Alvarez-Galván, M. C.; Vaquero, F.; Arenales, J.; Fierro, J. L. G. Hydrogen Production from Water Splitting Using Photo-Semiconductor Catalysts. In Renewable Hydrogen Technologies; Elsevier, 2013; pp 43-61. 
(12) Trotochaud, L.; Mills, T. J.; Boettcher, S. W. An Optocatalytic Model for Semiconductor-Catalyst Water-Splitting Photoelectrodes Based on In Situ Optical Measurements on Operational Catalysts. J. Phys. Chem. Lett. 2013, 4, 931-935.

(13) Gregoire, J. M.; Xiang, C.; Mitrovic, S.; Liu, X.; Marcin, M.; Cornell, E. W.; Fan, J.; Jin, J. Combined Catalysis and Optical Screening for High Throughput Discovery of Solar Fuels Catalysts. J. Electrochem. Soc. 2013, 160, F337-F342.

(14) Surendranath, Y.; Bediako, D. K.; Nocera, D. G. Interplay of Oxygen-Evolution Kinetics and Photovoltaic Power Curves on the Construction of Artificial Leaves. Proc. Natl. Acad. Sci. U. S. A. 2012, 109, 15617-15621.

(15) Vesborg, P. C. K.; Jaramillo, T. F. Addressing the Terawatt Challenge: Scalability in the Supply of Chemical Elements for Renewable Energy. RSC Adv. 2012, 2, 7933.

(16) Hong, W. T.; Risch, M.; Stoerzinger, K. a.; Grimaud, A.; Suntivich, J.; Shao-horn, Y. Toward the Rational Design of Non-Precious Transition Metal Oxides for Oxygen Electrocatalysis. Energy Environ. Sci. 2015, 13-15.

(17) Galán-Mascarós, J. R. Water Oxidation at Electrodes Modified with EarthAbundant Transition-Metal Catalysts. ChemElectroChem 2015, 2, 37-50.

(18) Gong, M.; Dai, H. A Mini Review of NiFe-Based Materials as Highly Active Oxygen Evolution Reaction Electrocatalysts. Nano Res. 2015, 8, 23-39.

(19) Trotochaud, L.; Ranney, J. K.; Williams, K. N.; Boettcher, S. W. Solution-Cast Metal Oxide Thin Film Electrocatalysts for Oxygen Evolution. J. Am. Chem. Soc. 2012, 134, 17253-17261.

(20) Burke, M. S.; Enman, L. J.; Batchellor, A. S.; Zou, S.; Boettcher, S. W. Oxygen Evolution Reaction Electrocatalysis on Transition Metal Oxides and (Oxy)hydroxides: Activity Trends and Design Principles. Chem. Mater. 2015, 27, 7549-7558.

(21) Haber, J. A.; Cai, Y.; Jung, S.; Xiang, C.; Mitrovic, S.; Jin, J.; Bell, A. T.; Gregoire, J. M. Discovering Ce-Rich Oxygen Evolution Catalysts, from High Throughput Screening to Water Electrolysis. Energy Environ. Sci. 2014, 7, 682.

(22) Burke, M. S.; Kast, M. G.; Trotochaud, L.; Smith, A. M.; Boettcher, S. W. CobaltIron (Oxy)hydroxide Oxygen Evolution Electrocatalysts: The Role of Structure and Composition on Activity, Stability, and Mechanism. J. Am. Chem. Soc. 2015, 137, 3638-3648. 
(23) Friebel, D.; Louie, M. W.; Bajdich, M.; Sanwald, K. E.; Cai, Y.; Wise, A. M.; Cheng, M.-J.; Sokaras, D.; Weng, T.-C.; Alonso-Mori, R.; et al. Identification of Highly Active Fe Sites in (Ni,Fe)OOH for Electrocatalytic Water Splitting. J. Am. Chem. Soc. 2015, 137, 1305-1313.

(24) Burke, M. S.; Zou, S.; Enman, L. J.; Kellon, J. E.; Gabor, C. A.; Pledger, E.; Boettcher, S. W. Revised Oxygen Evolution Reaction Activity Trends for FirstRow Transition-Metal (Oxy)hydroxides in Alkaline Media. J. Phys. Chem. Lett. 2015, 6, 3737-3742.

(25) Rong, X.; Parolin, J.; Kolpak, A. M. A Fundamental Relationship between Reaction Mechanism and Stability in Metal Oxide Catalysts for Oxygen Evolution. ACS Catal. 2016, 6, 1153-1158.

(26) Surendranath, Y.; Kanan, M. W.; Nocera, D. G. Mechanistic Studies of the Oxygen Evolution Reaction by a Cobalt-Phosphate Catalyst at Neutral pH. 2010, No. 14, 16501-16509.

(27) Corrigan, D. A.; Bendert, R. M. Effect of Coprecipitated Metal Ions on the Electrochemistry of Nickel Hydroxide Thin Films: Cyclic Voltammetry in $1 \mathrm{M}$ KOH. J. Electrochem. Soc. 1989, 136, 723-728.

(28) Smith, A. M.; Trotochaud, L.; Burke, M. S.; Boettcher, S. W. Contributions to Activity Enhancement via Fe Incorporation in Ni-(Oxy)hydroxide/borate Catalysts for near-Neutral pH Oxygen Evolution. Chem. Commun. 2015, 51, 5261-5263.

(29) El Baydi, M.; Tiwari, S. K.; Singh, R. N.; Rehspringer, J.-L.; Chartier, P.; Koenig, J. F.; Poillerat, G. High Specific Surface Area Nickel Mixed Oxide Powders $\mathrm{LaNiO}_{3}$ (Perovskite) and $\mathrm{NiCo}_{2} \mathrm{O}_{4}$ (Spinel) via Sol-Gel Type Routes for Oxygen Electrocatalysis in Alkaline Media. J. Solid State Chem. 1995, 116, 157-169.

(30) Gao, M.; Sheng, W.; Zhuang, Z.; Fang, Q.; Gu, S.; Jiang, J.; Yan, Y. Efficient Water Oxidation Using Nanostructured $\alpha$-Nickel-Hydroxide as an Electrocatalyst. J. Am. Chem. Soc. 2014, 136, 7077-7084.

(31) Fominykh, K.; Chernev, P.; Zaharieva, I.; Sicklinger, J.; Stefanic, G.; Döblinger, M.; Müller, A.; Pokharel, A.; Böcklein, S.; Scheu, C.; et al. Iron-Doped Nickel Oxide Nanocrystals as Highly Efficient Electrocatalysts for Alkaline Water Splitting. ACS Nano 2015, 9, 5180-5188.

(32) Gong, M.; Li, Y.; Wang, H.; Liang, Y.; Wu, J. Z.; Zhou, J.; Wang, J.; Regier, T.; Wei, F.; Dai, H. An Advanced Ni-Fe Layered Double Hydroxide Electrocatalyst for Water Oxidation. J. Am. Chem. Soc. 2013, 135, 8452-8455. 
(33) Tang, C.; Cheng, N.; Pu, Z.; Xing, W.; Sun, X. NiSe Nanowire Film Supported on Nickel Foam: An Efficient and Stable 3D Bifunctional Electrode for Full Water Splitting. Angew. Chem. Int. Ed. Engl. 2015, 54, 9351-9355.

(34) Stern, L.-A.; Feng, L.; Song, F.; Hu, X. Ni ${ }_{2} \mathrm{P}$ as a Janus Catalyst for Water Splitting: The Oxygen Evolution Activity of $\mathrm{Ni}_{2} \mathrm{P}$ Nanoparticles. Energy Environ. Sci. 2015, 8, 2347-2351.

(35) Zhou, W.; Wu, X.-J.; Cao, X.; Huang, X.; Tan, C.; Tian, J.; Liu, H.; Wang, J.; Zhang, $\mathrm{H}$. $\mathrm{Ni}_{3} \mathrm{~S}_{2}$ nanorods/Ni Foam Composite Electrode with Low Overpotential for Electrocatalytic Oxygen Evolution. Energy Environ. Sci. 2013, 6, 2921.

(36) Bediako, D. K.; Lassalle-Kaiser, B.; Surendranath, Y.; Yano, J.; Yachandra, V. K.; Nocera, D. G. Structure-Activity Correlations in a Nickel-Borate Oxygen Evolution Catalyst. J. Am. Chem. Soc. 2012, 134, 6801-6809.

(37) Schweitzer, George K., Pesterfield, L. L. The Aqueous Chemistry of the Elements; Oxford University Press: Oxford, 2010.

(38) Bode, H.; Dehmelt, K.; Witte, J. Zur Kenntnis Der nickelhydroxidelektrodeI.Über Das Nickel (II)-Hydroxidhydrat. Electrochim. Acta 1966, 11, 1079-1087.

(39) Van der Ven, A.; Morgan, D.; Meng, Y. S.; Ceder, G. Phase Stability of Nickel Hydroxides and Oxyhydroxides. J. Electrochem. Soc. 2006, 153, A210.

(40) Trotochaud, L.; Young, S. L.; Ranney, J. K.; Boettcher, S. W. Nickel-Iron Oxyhydroxide Oxygen-Evolution Electrocatalysts: The Role of Intentional and Incidental Iron Incorporation. J. Am. Chem. Soc. 2014, 136, 6744-6753.

(41) Oliva, P.; Leonardi, J.; Laurent, J. F.; Delmas, C.; Braconnier, J. J.; Figlarz, M.; Fievet, F.; de Guibert, A. Review of the Structure and the Electrochemistry of Nickel Hydroxides and Oxy-Hydroxides. J. Power Sources 1982, 8, 229-255.

(42) Klaus, S.; Cai, Y.; Louie, M. W.; Trotochaud, L.; Bell, A. T. Effects of Fe Electrolyte Impurities on $\mathrm{Ni}(\mathrm{OH})_{2} / \mathrm{NiOOH}$ Structure and Oxygen Evolution Activity. J. Phys. Chem. C 2015, 119, 7243-7254.

(43) Mellsop, S. R.; Gardiner, A.; Johannessen, B.; Marshall, A. T. Structure and Transformation of Oxy-Hydroxide Films on Ni Anodes below and above the Oxygen Evolution Potential in Alkaline Electrolytes. Electrochim. Acta 2015, 168, 356-364.

(44) Huggins, R. Positive Electrodes in Aqueous Systems. Adv. Batter. Mater. Sci. Asp. 2009, 213-234. 
(45) Landon, J.; Demeter, E.; İnoğlu, N.; Keturakis, C.; Wachs, I. E.; Vasić, R.;

Frenkel, A. I.; Kitchin, J. R. Spectroscopic Characterization of Mixed Fe-Ni Oxide Electrocatalysts for the Oxygen Evolution Reaction in Alkaline Electrolytes. ACS Catal. 2012, 2, 1793-1801.

(46) Enman, L. J.; Burke, M. S.; Batchellor, A. S.; Boettcher, S. W. Effects of Intentionally Incorporated Metal Cations on the Oxygen Evolution Electrocatalytic Activity of Nickel (Oxy)hydroxide in Alkaline Media. ACS Catal. 2016, 6, 24162423.

(47) Corrigan, D. A. The Catalysis of the Oxygen Evolution Reaction by Iron Impurities in Thin Film Nickel Oxide Electrodes. J. Electrochem. Soc. 1987, 134, $377-384$.

(48) Louie, M. W.; Bell, A. T. An Investigation of Thin-Film Ni-Fe Oxide Catalysts for the Electrochemical Evolution of Oxygen. J. Am. Chem. Soc. 2013, 135, 1232912337.

(49) Klaus, S.; Louie, M. W.; Trotochaud, L.; Bell, A. T. Role of Catalyst Preparation on the Electrocatalytic Activity of $\mathrm{Ni}_{(1-\mathrm{x})} \mathrm{Fe}_{\mathrm{x}} \mathrm{OOH}$ for the Oxygen Evolution Reaction. J. Phys. Chem. C 2015, 119, 18303-18316.

(50) Smith, R. D. L.; Prévot, M. S.; Fagan, R. D.; Trudel, S.; Berlinguette, C. P. Water Oxidation Catalysis: Electrocatalytic Response to Metal Stoichiometry in Amorphous Metal Oxide Films Containing Iron, Cobalt, and Nickel. J. Am. Chem. Soc. 2013, 135, 11580-11586.

(51) Bates, M. K.; Jia, Q.; Doan, H.; Liang, W.; Mukerjee, S. Charge-Transfer Effects in Ni-Fe \& Ni-Fe-Co Mixed-Metal-Oxides for the Alkaline Oxygen Evolution Reaction. ACS Catal. 2015, 6, 155-161.

(52) Fan, G.; Li, F.; Evans, D. G.; Duan, X. Catalytic Applications of Layered Double Hydroxides: Recent Advances and Perspectives. Chem. Soc. Rev. 2014, 43, 70407066.

(53) Batchellor, A. S.; Boettcher, S. W. Pulse-Electrodeposited Ni-Fe (Oxy)hydroxide Oxygen Evolution Electrocatalysts with High Geometric and Intrinsic Activities at Large Mass Loadings. ACS Catal. 2015, 5, 6680-6689.

(54) Bediako, D. K.; Costentin, C.; Jones, E. C.; Nocera, D. G.; Savéant, J.-M. ProtonElectron Transport and Transfer in Electrocatalytic Films. Application to a CobaltBased $\mathrm{O}_{2}$-Evolution Catalyst. J. Am. Chem. Soc. 2013, 135, 10492-10502.

(55) Morales-Guio, C. G.; Mayer, M. T.; Yella, A.; Tilley, S. D.; Grätzel, M.; Hu, X. An Optically Transparent Iron Nickel Oxide Catalyst for Solar Water Splitting. $J$. Am. Chem. Soc. 2015, 137, 9927-9936. 
(56) Egami, T.; Billinge, S. J. L. Underneath the Bragg Peaks Structural Analysis of Complex Materials; Pergamon, 2012.

(57) Billinge, S. J. L.; Kanatzidis, M. G. Beyond Crystallography: The Study of Disorder, Nanocrystallinity and Crystallographically Challenged Materials with Pair Distribution Functions. Chem. Commun. (Camb). 2004, 7, 749-760.

(58) Li, Z. Q.; Lu, C. J.; Xia, Z. P.; Zhou, Y.; Luo, Z. X-Ray Diffraction Patterns of Graphite and Turbostratic Carbon. Carbon N. Y. 2007, 45, 1686-1695.

(59) Görlin, M.; Chernev, P.; Ferreira de Araújo, J.; Reier, T.; Dresp, S.; Paul, B.; Krähnert, R.; Dau, H.; Strasser, P. Oxygen Evolution Reaction Dynamics, Faradaic Charge Efficiency, and the Active Metal Redox States of Ni-Fe Oxide Water Splitting Electrocatalysts. J. Am. Chem. Soc. 2016, 138, 5603-5614.

(60) Risch, M.; Klingan, K.; Heidkamp, J.; Ehrenberg, D.; Chernev, P.; Zaharieva, I.; Dau, H. Nickel-Oxido Structure of a Water-Oxidizing Catalyst Film. Chem. Commun. (Camb). 2011, 47, 11912-11914.

(61) Kwon, G.; Kokhan, O.; Han, A.; Chapman, K. W.; Chupas, P. J.; Du, P.; Tiede, D. M. Oxyanion Induced Variations in Domain Structure for Amorphous Cobalt Oxide Oxygen Evolving Catalysts, Resolved by X-Ray Pair Distribution Function Analysis. Acta Crystallogr. B. Struct. Sci. Cryst. Eng. Mater. 2015, 71, 713-721.

(62) Huang, J.; Blakemore, J. D.; Fazi, D.; Kokhan, O.; Schley, N. D.; Crabtree, R. H.; Brudvig, G. W.; Tiede, D. M. Domain Structure for an Amorphous Iridium-Oxide Water-Oxidation Catalyst Characterized by X-Ray Pair Distribution Function Analysis. Phys. Chem. Chem. Phys. 2014, 16, 1814-1819.

(63) Carteret, C.; Gregoire, B.; Ruby, C. Tunable Composition of $\mathrm{Ni}^{\mathrm{II}}-\mathrm{Al}^{\mathrm{III}}$ and $\mathrm{Ni}^{\mathrm{II}}-$ $\mathrm{Fe}^{\mathrm{III}}$ Layered Hydroxides within a Wide Range of Layer Charge. Solid State Sci. 2011, 13, 146-150.

(64) Qiu, X.; Božin, E. S.; Juhas, P.; Proffen, T.; Billinge, S. J. L.; IUCr; G., D. R.; H., K. G.; J., N. J.; D., T. J.; et al. Reciprocal-Space Instrumental Effects on the RealSpace Neutron Atomic Pair Distribution Function. J. Appl. Crystallogr. 2004, 37, $110-116$.

(65) Cui, B.; Lin, H.; Li, J. B.; Li, X.; Yang, J.; Tao, J. Core-Ring Structured $\mathrm{NiCo}_{2} \mathrm{O}_{4}$ Nanoplatelets: Synthesis, Characterization, and Electrocatalytic Applications. Adv. Funct. Mater. 2008, 18, 1441-1447.

(66) Faour, A.; Mousty, C.; Prevot, V.; Devouard, B.; De Roy, A.; Bordet, P.; Elkaim, E.; Taviot-Gueho, C. Correlation among Structure, Microstructure, and Electrochemical Properties of NiAl- $\mathrm{CO}_{3}$ Layered Double Hydroxide Thin Films. J. Phys. Chem. C 2012, 116, 15646-15659. 


\section{Chapter II}

References

(1) Galán-Mascarós, J. R. Water Oxidation at Electrodes Modified with EarthAbundant Transition-Metal Catalysts. ChemElectroChem 2015, 2, 37-50.

(2) Wang, M.; Wang, Z.; Gong, X.; Guo, Z. The Intensification Technologies to Water Electrolysis for Hydrogen Production - A Review. Renew. Sustain. Energy Rev.

2014, 29, 573-588.

(3) Herrero, C.; Lassallekaiser, B.; Leibl, W.; Rutherford, A.; Aukauloo, A. Artificial Systems Related to Light Driven Electron Transfer Processes in PSII. Coord. Chem. Rev. 2008, 252, 456-468.

(4) Gregoire, J. M.; Xiang, C.; Mitrovic, S.; Liu, X.; Marcin, M.; Cornell, E. W.; Fan, J.; Jin, J. Combined Catalysis and Optical Screening for High Throughput Discovery of Solar Fuels Catalysts. J. Electrochem. Soc. 2013, 160, F337-F342.

(5) McCrory, C. C. L.; Jung, S.; Peters, J. C.; Jaramillo, T. F. Benchmarking Heterogeneous Electrocatalysts for the Oxygen Evolution Reaction. J. Am. Chem. Soc. 2013, 135, 16977-16987.

(6) Smith, R. D. L.; Prévot, M. S.; Fagan, R. D.; Trudel, S.; Berlinguette, C. P. Water Oxidation Catalysis: Electrocatalytic Response to Metal Stoichiometry in Amorphous Metal Oxide Films Containing Iron, Cobalt, and Nickel. J. Am. Chem. Soc. 2013, 135, 11580-11586.

(7) Trotochaud, L.; Young, S. L.; Ranney, J. K.; Boettcher, S. W. Nickel-Iron Oxyhydroxide Oxygen-Evolution Electrocatalysts: The Role of Intentional and Incidental Iron Incorporation. J. Am. Chem. Soc. 2014, 136, 6744-6753.

(8) Angelinetta, C.; Trasatti, S.; Atanasoska, L. D.; Minevski, Z. S.; Atanasoski, R. T. Effect of Preparation on the Surface and Electrocatalytic Properties of $\mathrm{RuO}_{2}+\mathrm{IrO}_{2}$ Mixed Oxide Electrodes. Mater. Chem. Phys. 1989, 22, 231-247.

(9) Ayers, K. E.; Anderson, E. B.; Capuano, C.; Carter, B.; Dalton, L.; Hanlon, G.; Manco, J.; Niedzwiecki, M. Research Advances towards Low Cost, High Efficiency PEM Electrolysis. ECS Trans. 2010, 33, 3-15.

(10) Hall, D. E. Alkaline Water Electrolysis Anode Materials. J. Electrochem. Soc. 1985, 132, 41C-48C.

(11) Gong, M.; Dai, H. A Mini Review of NiFe-Based Materials as Highly Active Oxygen Evolution Reaction Electrocatalysts. Nano Res. 2014, 8, 23-39. 
(12) Wendt, H.; Kreysa, G. Electrochemical Engineering: Science and Technology in Chemical and Other Industries, 1st ed.; Springer: Berlin, 1999.

(13) Lu, X.; Zhao, C. Electrodeposition of Hierarchically Structured ThreeDimensional Nickel-iron Electrodes for Efficient Oxygen Evolution at High Current Densities. Nat. Commun. 2015, 6, 1-7.

(14) Pérez-Alonso, F. J.; Adán, C.; Rojas, S.; Peña, M. a.; Fierro, J. L. G. Ni/Fe Electrodes Prepared by Electrodeposition Method over Different Substrates for Oxygen Evolution Reaction in Alkaline Medium. Int. J. Hydrogen Energy 2014, 39, 5204-5212.

(15) Ma, W.; Ma, R.; Wang, C.; Liang, J.; Liu, X.; Zhou, K.; Sasaki, T. A Superlattice of Alternately Stacked Ni-Fe Hydroxide Nanosheets and Graphene for Efficient Splitting of Water. ACS Nano 2015, 9, 1977-1984.

(16) Gong, M.; Li, Y.; Wang, H.; Liang, Y.; Wu, J. Z.; Zhou, J.; Wang, J.; Regier, T.; Wei, F.; Dai, H. An Advanced Ni-Fe Layered Double Hydroxide Electrocatalyst for Water Oxidation. J. Am. Chem. Soc. 2013, 135, 8452-8455.

(17) Marini, S.; Salvi, P.; Nelli, P.; Pesenti, R.; Villa, M.; Kiros, Y. Oxygen Evolution in Alkali with Gas Diffusion Electrodes. Int. J. Hydrogen Energy 2013, 38, 11496-11506.

(18) Gao, M.; Sheng, W.; Zhuang, Z.; Fang, Q.; Gu, S.; Jiang, J.; Yan, Y. Efficient Water Oxidation Using Nanostructured $\alpha$-Nickel-Hydroxide as an Electrocatalyst. J. Am. Chem. Soc. 2014, 136, 7077-7084.

(19) Hunter, B. M.; Blakemore, J. D.; Deimund, M.; Gray, H. B.; Winkler, J. R.; Müller, A. M. Highly Active Mixed-Metal Nanosheet Water Oxidation Catalysts Made by Pulsed-Laser Ablation in Liquids. J. Am. Chem. Soc. 2014, 136, 13118 13121.

(20) Doyle, R. L.; Godwin, I. J.; Brandon, M. P.; Lyons, M. E. G. Redox and Electrochemical Water Splitting Catalytic Properties of Hydrated Metal Oxide Modified Electrodes. Phys. Chem. Chem. Phys. 2013, 15, 13737-13783.

(21) Trotochaud, L.; Ranney, J. K.; Williams, K. N.; Boettcher, S. W. Solution-Cast Metal Oxide Thin Film Electrocatalysts for Oxygen Evolution. J. Am. Chem. Soc. 2012, 134, 17253-17261.

(22) Klingan, K.; Ringleb, F.; Zaharieva, I.; Heidkamp, J.; Chernev, P.; GonzalezFlores, D.; Risch, M.; Fischer, A.; Dau, H. Water Oxidation by Amorphous Cobalt-Based Oxides: Volume Activity and Proton Transfer to Electrolyte Bases. ChemSusChem 2014, 7, 1-11. 
(23) May, K. J.; Carlton, C. E.; Stoerzinger, K. A.; Risch, M.; Suntivich, J.; Lee, Y.-L.; Grimaud, A.; Shao-Horn, Y. Influence of Oxygen Evolution during Water Oxidation on the Surface of Perovskite Oxide Catalysts. J. Phys. Chem. Lett. 2012, 3, 3264-3270.

(24) Bode, H.; Dehmelt, K.; Witte, J. Zur Kenntnis Der nickelhydroxidelektrodeI.Über Das Nickel (II)-Hydroxidhydrat. Electrochim. Acta 1966, 11, 1079-1087.

(25) Friebel, D.; Louie, M. W.; Bajdich, M.; Sanwald, K. E.; Cai, Y.; Wise, A. M.; Cheng, M.-J.; Sokaras, D.; Weng, T.-C.; Alonso-Mori, R.; et al. Identification of Highly Active Fe Sites in (Ni,Fe)OOH for Electrocatalytic Water Splitting. J. Am. Chem. Soc. 2015, 137, 1305-1313.

(26) Fan, G.; Li, F.; Evans, D. G.; Duan, X. Catalytic Applications of Layered Double Hydroxides: Recent Advances and Perspectives. Chem. Soc. Rev. 2014, 43, 70407066.

(27) Burke, M. S.; Zou, S.; Enman, L. J.; Kellon, J. E.; Gabor, C. A.; Pledger, E.; Boettcher, S. W. Revised Oxygen Evolution Reaction Activity Trends for FirstRow Transition-Metal (Oxy)hydroxides in Alkaline Media. J. Phys. Chem. Lett. 2015, 6, 3737-3742.

(28) Klaus, S.; Cai, Y.; Louie, M. W.; Trotochaud, L.; Bell, A. T. Effects of Fe Electrolyte Impurities on $\mathrm{Ni}(\mathrm{OH})_{2} / \mathrm{NiOOH}$ Structure and Oxygen Evolution Activity. J. Phys. Chem. C 2015, 119, 7243-7254.

(29) Burke, M. S.; Kast, M. G.; Trotochaud, L.; Smith, A. M.; Boettcher, S. W. CobaltIron (Oxy)hydroxide Oxygen Evolution Electrocatalysts: The Role of Structure and Composition on Activity, Stability, and Mechanism. J. Am. Chem. Soc. 2015, 137, 3638-3648.

(30) Burke, M. S.; Enman, L. J.; Batchellor, A. S.; Zou, S.; Boettcher, S. W. Oxygen Evolution Reaction Electrocatalysis on Transition Metal Oxides and (Oxy)hydroxides: Activity Trends and Design Principles. Chem. Mater. 2015, 27, 7549-7558.

(31) Yeo, B. S.; Bell, A. T. In Situ Raman Study of Nickel Oxide and Gold-Supported Nickel Oxide Catalysts for the Electrochemical Evolution of Oxygen. J. Phys. Chem. C 2012, 116, 8394-8400.

(32) Yeo, B. S.; Bell, A. T. Enhanced Activity of Gold-Supported Cobalt Oxide for the Electrochemical Evolution of Oxygen. J. Am. Chem. Soc. 2011, 133, 5587-5593.

(33) Bediako, D. K.; Costentin, C.; Jones, E. C.; Nocera, D. G.; Savéant, J.-M. ProtonElectron Transport and Transfer in Electrocatalytic Films. Application to a CobaltBased O2-Evolution Catalyst. J. Am. Chem. Soc. 2013, 135, 10492-10502. 
(34) Corrigan, D. A.; Bendert, R. M. Effect of Coprecipitated Metal Ions on the Electrochemistry of Nickel Hydroxide Thin Films: Cyclic Voltammetry in $1 \mathrm{M}$ KOH. J. Electrochem. Soc. 1989, 136, 723-728.

(35) Bouessay, I.; Rougier, A.; Poizot, P.; Moscovici, J.; Michalowicz, A.; Tarascon, J.M. Electrochromic Degradation in Nickel Oxide Thin Film: A Self-Discharge and Dissolution Phenomenon. Electrochim. Acta 2005, 50, 3737-3745.

(36) McCrory, C. C. L.; Jung, S.; Ferrer, I. M.; Chatman, S.; Peters, J. C.; Jaramillo, T. F. Benchmarking HER and OER Electrocatalysts for Solar Water Splitting Devices. J. Am. Chem. Soc. 2015, 137, 4347-4357.

(37) Wehrens-Dijksma, M.; Notten, P. H. L. Electrochemical Quartz Microbalance Characterization of $\mathrm{Ni}(\mathrm{OH}) 2-B a s e d$ Thin Film Electrodes. Electrochim. Acta 2006, 51, 3609-3621.

(38) Schumacher, R.; Borges, G.; Kanazawa, K. K. The Quartz Microbalance: A Sensitive Tool to Probe Surface Reconstructions on Gold Electrodes in Liquid. Surf. Sci. 1985, 163, L621-L626.

(39) Kim, H.-S.; Mora-Sero, I.; Gonzalez-Pedro, V.; Fabregat-Santiago, F.; JuarezPerez, E. J.; Park, N.-G.; Bisquert, J. Mechanism of Carrier Accumulation in Perovskite Thin-Absorber Solar Cells. Nat. Commun. 2013, 4, 2242.

(40) Weininger, J. L.; Breiter, M. W. Hydrogen Evolution and Surface Oxidation of Nickel Electrodes in Alkaline Solution. J. Electrochem. Soc. 1964, 111, 707-712.

(41) Hampson, N. A.; Latham, R. J.; Lee, J. B.; Macdonald, K. I. Oxidations at Copper Electrodes. J. Electroanal. Chem. Interfacial Electrochem. 1971, 31, 57-62.

(42) Lasia, A.; Rami, A. Kinetics of Hydrogen Evolution on Nickel Electrodes. $J$. Electroanal. Chem. Interfacial Electrochem. 1990, 294, 123-141.

(43) Gu, P.; Bai, L.; Gao, L.; Brousseau, R.; Conway, B. E. Problems in the Determination of Adsorption Behaviour of Intermediates in Faradaic Reactions: Distinction between Double Layer and Adsorption Capacitance of Electrocatalysts Determined from Fast Potential Relaxation Transients. Electrochim. Acta 1992, $37,2145-2154$.

(44) Wu, G.; Li, N.; Zhou, D. R.; Mitsuo, K.; Xu, B. Q. Anodically Electrodeposited Co+Ni Mixed Oxide Electrode: Preparation and Electrocatalytic Activity for Oxygen Evolution in Alkaline Media. J. Solid State Chem. 2004, 177, 3682-3692. 
(45) Gu, P.; Bai, L.; Gao, L.; Brousseau, R.; Conway, B. E. Problems in the Determination of Adsorption Behaviour of Intermediates in Faradaic Reactions: Distinction between Double Layer and Adsorption Capacitance of Electrocatalysts Determined from Fast Potential Relaxation Transients. Electrochim. Acta 1992, $37,2145-2154$.

(46) Fominykh, K.; Chernev, P.; Zaharieva, I.; Sicklinger, J.; Stefanic, G.; Döblinger, M.; Müller, A.; Pokharel, A.; Böcklein, S.; Scheu, C.; et al. Iron-Doped Nickel Oxide Nanocrystals as Highly Efficient Electrocatalysts for Alkaline Water Splitting. ACS Nano 2015, 9, 5180-5188.

(47) Schweitzer, George K., Pesterfield, L. L. The Aqueous Chemistry of the Elements; Oxford University Press: Oxford, 2010.

(48) Corrigan, D. A.; Knight, S. L. Electrochemical and Spectroscopic Evidence on the Participation of Quadrivalent Nickel in the Nickel Hydroxide Redox Reaction. $J$. Electrochem. Soc. 1989, 136, 613-619.

(49) Barnard, R.; Randell, C. F.; Tye, F. L. Studies Concerning Charged Nickel Hydroxide Electrodes I. Measurement of Reversible Potentials. J. Appl. Electrochem. 1980, 10, 109-125.

(50) Oliva, P.; Leonardi, J.; Laurent, J. F.; Delmas, C.; Braconnier, J. J.; Figlarz, M.; Fievet, F.; de Guibert, A. Review of the Structure and the Electrochemistry of Nickel Hydroxides and Oxy-Hydroxides. J. Power Sources 1982, 8, 229-255.

(51) Trotochaud, L.; Mills, T. J.; Boettcher, S. W. An Optocatalytic Model for Semiconductor-Catalyst Water-Splitting Photoelectrodes Based on In Situ Optical Measurements on Operational Catalysts. J. Phys. Chem. Lett. 2013, 4, 931-935.

(52) Millet, P.; Grigoriev, S. Renewable Hydrogen Technologies; Gandía, L. M., Arzamendi, G., Diéguez, P. M., Eds.; Elsevier: Amsterdam, 2013.

(53) Corrigan, D. A. The Catalysis of the Oxygen Evolution Reaction by Iron Impurities in Thin Film Nickel Oxide Electrodes. J. Electrochem. Soc. 1987, 134, 377-384.

(54) Louie, M. W.; Bell, A. T. An Investigation of Thin-Film Ni-Fe Oxide Catalysts for the Electrochemical Evolution of Oxygen. J. Am. Chem. Soc. 2013, 135, 1232912337.

(55) Smith, A. M.; Trotochaud, L.; Burke, M. S.; Boettcher, S. W. Contributions to Activity Enhancement via Fe Incorporation in Ni-(Oxy)hydroxide/borate Catalysts for near-Neutral pH Oxygen Evolution. Chem. Commun. 2015, 51, 5261-5263. 
(56) Strehblow, H.-H. Advances in Electrochemical Science and Engineering, Volume 8.; Alkire, Richard C., Kolb, D. M., Ed.; Wiley-VCH: Weinheim, 2003.

\section{Chapter III}

(1) Chen, H.; Cong, T. N.; Yang, W.; Tan, C.; Li, Y.; Ding, Y. Progress in Electrical Energy Storage System: A Critical Review. Prog. Nat. Sci. 2009, 19, 291-312.

(2) Cook, T. R.; Dogutan, D. K.; Reece, S. Y.; Surendranath, Y.; Teets, T. S.; Nocera, D. G. Solar Energy Supply and Storage for the Legacy and Nonlegacy Worlds. Chem. Rev. 2010, 110, 6474-6502.

(3) Poizot, P.; Dolhem, F. Clean Energy New Deal for a Sustainable World: From Non-CO2 Generating Energy Sources to Greener Electrochemical Storage Devices. Energy Environ. Sci. 2011, 4, 2003.

(4) Walter, M. G.; Warren, E. L.; McKone, J. R.; Boettcher, S. W.; Mi, Q.; Santori, E. A.; Lewis, N. S. Solar Water Splitting Cells. Chem. Rev. 2010, 110, 6446-6473.

(5) Galán-Mascarós, J. R. Water Oxidation at Electrodes Modified with EarthAbundant Transition-Metal Catalysts. ChemElectroChem 2015, 2, 37-50.

(6) Wang, M.; Wang, Z.; Gong, X.; Guo, Z. The Intensification Technologies to Water Electrolysis for Hydrogen Production - A Review. Renew. Sustain. Energy Rev. 2014, 29, 573-588.

(7) Trotochaud, L.; Ranney, J. K.; Williams, K. N.; Boettcher, S. W. Solution-Cast Metal Oxide Thin Film Electrocatalysts for Oxygen Evolution. J. Am. Chem. Soc. 2012, 134, 17253-17261.

(8) Hall, D. E. Alkaline Water Electrolysis Anode Materials. J. Electrochem. Soc. 1985, 132, 41C-48C.

(9) Zeng, K.; Zhang, D. Recent Progress in Alkaline Water Electrolysis for Hydrogen Production and Applications. Prog. Energy Combust. Sci. 2010, 36, 307-326.

(10) Marini, S.; Salvi, P.; Nelli, P.; Pesenti, R.; Villa, M.; Berrettoni, M.; Zangari, G.; Kiros, Y. Advanced Alkaline Water Electrolysis. Electrochim. Acta 2012, 82, 384391.

(11) Manabe, A.; Kashiwase, M.; Hashimoto, T.; Hayashida, T.; Kato, A.; Hirao, K.; Shimomura, I.; Nagashima, I. Basic Study of Alkaline Water Electrolysis. Electrochim. Acta 2013, 100, 249-256. 
(12) Xiao, L.; Zhang, S.; Pan, J.; Yang, C.; He, M.; Zhuang, L.; Lu, J. First Implementation of Alkaline Polymer Electrolyte Water Electrolysis Working Only with Pure Water. Energy Environ. Sci. 2012, 5, 7869.

(13) Corrigan, D. A. The Catalysis of the Oxygen Evolution Reaction by Iron Impurities in Thin Film Nickel Oxide Electrodes. J. Electrochem. Soc. 1987, 134, 377-384.

(14) Stern, L.-A.; Feng, L.; Song, F.; Hu, X. Ni ${ }_{2} \mathrm{P}$ as a Janus Catalyst for Water Splitting: The Oxygen Evolution Activity of $\mathrm{Ni}_{2} \mathrm{P}$ Nanoparticles. Energy Environ. Sci. 2015, 8, 2347-2351.

(15) Tang, C.; Cheng, N.; Pu, Z.; Xing, W.; Sun, X. NiSe Nanowire Film Supported on Nickel Foam: An Efficient and Stable 3D Bifunctional Electrode for Full Water Splitting. Angew. Chem. Int. Ed. Engl. 2015, 54, 9351-9355.

(16) Zhou, W.; Wu, X.-J.; Cao, X.; Huang, X.; Tan, C.; Tian, J.; Liu, H.; Wang, J.; Zhang, $\mathrm{H} . \mathrm{Ni}_{3} \mathrm{~S}_{2}$ nanorods/Ni Foam Composite Electrode with Low Overpotential for Electrocatalytic Oxygen Evolution. Energy Environ. Sci. 2013, 6, 2921.

(17) Burke, M. S.; Zou, S.; Enman, L. J.; Kellon, J. E.; Gabor, C. A.; Pledger, E.; Boettcher, S. W. Revised Oxygen Evolution Reaction Activity Trends for FirstRow Transition-Metal (Oxy)hydroxides in Alkaline Media. J. Phys. Chem. Lett. 2015, 6, 3737-3742.

(18) Smith, A. M.; Trotochaud, L.; Burke, M. S.; Boettcher, S. W. Contributions to Activity Enhancement via Fe Incorporation in Ni-(Oxy)hydroxide/borate Catalysts for near-Neutral pH Oxygen Evolution. Chem. Commun. 2015, 51, 5261-5263.

(19) Corrigan, D. A.; Bendert, R. M. Effect of Coprecipitated Metal Ions on the Electrochemistry of Nickel Hydroxide Thin Films: Cyclic Voltammetry in $1 \mathrm{M}$ KOH. J. Electrochem. Soc. 1989, 136, 723-728.

(20) Wu, G.; Li, N.; Zhou, D.-R.; Mitsuo, K.; Xu, B.-Q. Anodically Electrodeposited $\mathrm{Co}+\mathrm{Ni}$ Mixed Oxide Electrode: Preparation and Electrocatalytic Activity for Oxygen Evolution in Alkaline Media. J. Solid State Chem. 2004, 177, 3682-3692.

(21) El Baydi, M.; Tiwari, S. K.; Singh, R. N.; Rehspringer, J.-L.; Chartier, P.; Koenig, J. F.; Poillerat, G. High Specific Surface Area Nickel Mixed Oxide Powders $\mathrm{LaNiO}_{3}$ (Perovskite) and $\mathrm{NiCo}_{2} \mathrm{O}_{4}$ (Spinel) via Sol-Gel Type Routes for Oxygen Electrocatalysis in Alkaline Media. J. Solid State Chem. 1995, 116, 157-169.

(22) Gao, M.; Sheng, W.; Zhuang, Z.; Fang, Q.; Gu, S.; Jiang, J.; Yan, Y. Efficient Water Oxidation Using Nanostructured $\alpha$-Nickel-Hydroxide as an Electrocatalyst. J. Am. Chem. Soc. 2014, 136, 7077-7084. 
(23) Fominykh, K.; Chernev, P.; Zaharieva, I.; Sicklinger, J.; Stefanic, G.; Döblinger, M.; Müller, A.; Pokharel, A.; Böcklein, S.; Scheu, C.; et al. Iron-Doped Nickel Oxide Nanocrystals as Highly Efficient Electrocatalysts for Alkaline Water Splitting. ACS Nano 2015, 9, 5180-5188.

(24) Gong, M.; Li, Y.; Wang, H.; Liang, Y.; Wu, J. Z.; Zhou, J.; Wang, J.; Regier, T.; Wei, F.; Dai, H. An Advanced Ni-Fe Layered Double Hydroxide Electrocatalyst for Water Oxidation. J. Am. Chem. Soc. 2013, 135, 8452-8455.

(25) Liang, H.; Meng, F.; Cabán-Acevedo, M.; Li, L.; Forticaux, A.; Xiu, L.; Wang, Z.; Jin, S. Hydrothermal Continuous Flow Synthesis and Exfoliation of NiCo Layered Double Hydroxide Nanosheets for Enhanced Oxygen Evolution Catalysis. Nano Lett. 2015, 15, 1421-1427.

(26) Xu, Y.; Hao, Y.; Zhang, G.; Lu, Z.; Han, S.; Li, Y.; Sun, X. Room-Temperature Synthetic NiFe Layered Double Hydroxide with Different Anions Intercalation as an Excellent Oxygen Evolution Catalyst. RSC Adv. 2015, 5, 55131-55135.

(27) Smith, R. D. L.; Prévot, M. S.; Fagan, R. D.; Zhang, Z.; Sedach, P. A.; Siu, M. K. J.; Trudel, S.; Berlinguette, C. P. Photochemical Route for Accessing Amorphous Metal Oxide Materials for Water Oxidation Catalysis. Science 2013, 340, 60-63.

(28) Schweitzer, George K., Pesterfield, L. L. The Aqueous Chemistry of the Elements; Oxford University Press: Oxford, 2010.

(29) Binninger, T.; Mohamed, R.; Waltar, K.; Fabbri, E.; Levecque, P.; Kötz, R.; Schmidt, T. J. Thermodynamic Explanation of the Universal Correlation between Oxygen Evolution Activity and Corrosion of Oxide Catalysts. Sci. Rep. 2015, 5, 12167.

(30) Lyons, M. E. G.; Russell, L.; O’Brien, M.; Doyle, R. L.; Godwin, I.; Brandon, M. P. Redox Switching and Oxygen Evolution at Hydrous Oxyhydroxide Modified Nickel Electrodes in Aqueous Alkaline Solution: Effect of Hydrous Oxide Thickness and Base Concentration. Int. J. Electrochem. Sci. 2012, 7, 2710-2763.

(31) Bergmann, A.; Martinez-Moreno, E.; Teschner, D.; Chernev, P.; Gliech, M.; de Araújo, J. F.; Reier, T.; Dau, H.; Strasser, P. Reversible Amorphization and the Catalytically Active State of Crystalline $\mathrm{Co}_{3} \mathrm{O}_{4}$ during Oxygen Evolution. Nat. Commun. 2015, 6, 8625.

(32) Trotochaud, L.; Young, S. L.; Ranney, J. K.; Boettcher, S. W. Nickel-Iron Oxyhydroxide Oxygen-Evolution Electrocatalysts: The Role of Intentional and Incidental Iron Incorporation. J. Am. Chem. Soc. 2014, 136, 6744-6753. 
(33) Burke, M. S.; Enman, L. J.; Batchellor, A. S.; Zou, S.; Boettcher, S. W. Oxygen Evolution Reaction Electrocatalysis on Transition Metal Oxides and (Oxy)hydroxides: Activity Trends and Design Principles. Chem. Mater. 2015, 27, 7549-7558.

(34) Friebel, D.; Louie, M. W.; Bajdich, M.; Sanwald, K. E.; Cai, Y.; Wise, A. M.; Cheng, M.-J.; Sokaras, D.; Weng, T.-C.; Alonso-Mori, R.; et al. Identification of Highly Active Fe Sites in (Ni,Fe)OOH for Electrocatalytic Water Splitting. J. Am. Chem. Soc. 2015, 137, 1305-1313.

(35) Goerlin, M.; Chernev, P.; Ferreira de Araújo, J.; Reier, T.; Dresp, S.; Paul, B.; Kraehnert, R.; Dau, H.; Strasser, P. Oxygen Evolution Reaction Dynamics, Faradaic Charge Efficiency, and the Active Metal Redox States of Ni-Fe Oxide Water Splitting Electrocatalysts. J. Am. Chem. Soc. 2016, 138, 5603-5614.

(36) Chen, J. Y. C.; Dang, L.; Liang, H.; Bi, W.; Gerken, J. B.; Jin, S.; Alp, E. E.; Stahl, S. S. Operando Analysis of NiFe and $\mathrm{Fe}$ Oxyhydroxide Electrocatalysts for Water Oxidation: Detection of Fe4+ by Mössbauer Spectroscopy. J. Am. Chem. Soc. 2015, 137, 15090-15093.

(37) Hunter, B. M.; Blakemore, J. D.; Deimund, M.; Gray, H. B.; Winkler, J. R.; Müller, A. M. Highly Active Mixed-Metal Nanosheet Water Oxidation Catalysts Made by Pulsed-Laser Ablation in Liquids. J. Am. Chem. Soc. 2014, 136, 13118 13121.

(38) Bediako, D. K.; Lassalle-Kaiser, B.; Surendranath, Y.; Yano, J.; Yachandra, V. K.; Nocera, D. G. Structure-Activity Correlation in a Nickel-Borate Oxygen Evolution Catalyst. 2012, 134, 6801-6809.

(39) Risch, M.; Klingan, K.; Heidkamp, J.; Ehrenberg, D.; Chernev, P.; Zaharieva, I.; Dau, H. Nickel-Oxido Structure of a Water-Oxidizing Catalyst Film. Chem. Commun. (Camb). 2011, 47, 11912-11914.

(40) Bates, M. K.; Jia, Q.; Doan, H.; Liang, W.; Mukerjee, S. Charge-Transfer Effects in Ni-Fe \& Ni-Fe-Co Mixed-Metal-Oxides for the Alkaline Oxygen Evolution Reaction. ACS Catal. 2015, 6, 155-161.

(41) Zhang, C.; Fagan, R. D.; Smith, R. D. L.; Moore, S. A.; Berlinguette, C. P.; Trudel, S. Mapping the Performance of Amorphous Ternary Metal Oxide Water Oxidation Catalysts Containing Aluminium. J. Mater. Chem. A 2015, 3, 756-761.

(42) Haber, J. A.; Anzenburg, E.; Yano, J.; Kisielowski, C.; Gregoire, J. M. Multiphase Nanostructure of a Quinary Metal Oxide Electrocatalyst Reveals a New Direction for OER Electrocatalyst Design. Adv. Energy Mater. 2015, 5,1402307. 
(43) Bajdich, M.; García-Mota, M.; Vojvodic, A.; Nørskov, J. K.; Bell, A. T. Theoretical Investigation of the Activity of Cobalt Oxides for the Electrochemical Oxidation of Water. J. Am. Chem. Soc. 2013, 135, 13521-13530.

(44) Pérez-Alonso, F. J.; Adán, C.; Rojas, S.; Peña, M. a.; Fierro, J. L. G. Ni/Fe Electrodes Prepared by Electrodeposition Method over Different Substrates for Oxygen Evolution Reaction in Alkaline Medium. Int. J. Hydrogen Energy 2014, 39, 5204-5212.

(45) Van Drunen, J.; Kinkead, B.; Wang, M. C. P.; Sourty, E.; Gates, B. D.; Jerkiewicz, G. Comprehensive Structural, Surface-Chemical and Electrochemical Characterization of Nickel-Based Metallic Foams. ACS Appl. Mater. Interfaces 2013, 5, 6712-6722.

(46) Carteret, C.; Gregoire, B.; Ruby, C. Tunable Composition of Ni ${ }^{\mathrm{II}}-\mathrm{Al}^{\mathrm{III}}$ and $\mathrm{Ni}^{\mathrm{II}}-$ $\mathrm{Fe}^{\mathrm{III}}$ Layered Hydroxides within a Wide Range of Layer Charge. Solid State Sci. 2011, 13, 146-150.

(47) Wu, M.-S. S.; Huang, K.-C. C. Enhanced Electrochemical Performance of Nickel Hydroxide Electrode with Monolayer Hollow Spheres Composed of Nanoflakes. Int. J. Hydrogen Energy 2011, 36, 13407-13413.

(48) Billinge, S. J. L.; Kanatzidis, M. G. Beyond Crystallography: The Study of Disorder, Nanocrystallinity and Crystallographically Challenged Materials with Pair Distribution Functions. Chem. Commun. (Camb). 2004, No. 7, 749-760.

(49) Kwon, G.; Kokhan, O.; Han, A.; Chapman, K. W.; Chupas, P. J.; Du, P.; Tiede, D. M. Oxyanion Induced Variations in Domain Structure for Amorphous Cobalt Oxide Oxygen Evolving Catalysts, Resolved by X-Ray Pair Distribution Function Analysis. Acta Crystallogr. B. Struct. Sci. Cryst. Eng. Mater. 2015, 71, 713-721.

(50) Farrow, C. L.; Bediako, D. K.; Surendranath, Y.; Nocera, D. G.; Billinge, S. J. L. Intermediate-Range Structure of Self-Assembled Cobalt-Based Oxygen-Evolving Catalyst. J. Am. Chem. Soc. 2013, 135, 6403-6406.

(51) Du, P.; Kokhan, O.; Chapman, K. W.; Chupas, P. J.; Tiede, D. M. Elucidating the Domain Structure of the Cobalt Oxide Water Splitting Catalyst by X-Ray Pair Distribution Function Analysis. J. Am. Chem. Soc. 2012, 134, 11096-11099.

(52) Kurzman, J. A.; Dettelbach, K. E.; Martinolich, A. J.; Berlinguette, C. P.; Neilson, J. R. Structural Characteristics and Eutaxy in the Photo-Deposited Amorphous Iron Oxide Oxygen Evolution Catalyst. Chem. Mater. 2015, 27, 3462-3470.

(53) Huynh, M.; Shi, C.; Billinge, S. J. L.; Nocera, D. G. Nature of Activated Manganese Oxide for Oxygen Evolution. J. Am. Chem. Soc. 2015, 137, 1488714904. 
(54) Huang, J.; Blakemore, J. D.; Fazi, D.; Kokhan, O.; Schley, N. D.; Crabtree, R. H.; Brudvig, G. W.; Tiede, D. M. Domain Structure for an Amorphous Iridium-Oxide Water-Oxidation Catalyst Characterized by X-Ray Pair Distribution Function Analysis. Phys. Chem. Chem. Phys. 2014, 16, 1814-1819.

(55) Batchellor, A. S.; Boettcher, S. W. Pulse-Electrodeposited Ni-Fe (Oxy)hydroxide Oxygen Evolution Electrocatalysts with High Geometric and Intrinsic Activities at Large Mass Loadings. ACS Catal. 2015, 5, 6680-6689.

(56) Qiu, X.; Thompson, J. W.; Billinge, S. J. L. PDFgetX2 : A GUI-Driven Program to Obtain the Pair Distribution Function from X-Ray Powder Diffraction Data. $J$. Appl. Crystallogr. 2004, 37, 678-678.

(57) Juhás, P.; Davis, T.; Farrow, C. L.; Billinge, S. J. L. PDFgetX3 : A Rapid and Highly Automatable Program for Processing Powder Diffraction Data into Total Scattering Pair Distribution Functions. J. Appl. Crystallogr. 2013, 46, 560-566.

(58) Zuo, X.; Cui, G.; Merz, K. M.; Zhang, L.; Lewis, F. D.; Tiede, D. M. X-Ray Diffraction "fingerprinting" of DNA Structure in Solution for Quantitative Evaluation of Molecular Dynamics Simulation. Proc. Natl. Acad. Sci. U. S. A. 2006, 103, 3534-3539.

(59) Zhang, L. Y.; Friesner, R. A. Ab Initio Calculation of Electronic Coupling in the Photosynthetic Reaction Center. Proc. Natl. Acad. Sci. 1998, 95, 13603-13605.

(60) Bode, H.; Dehmelt, K.; Witte, J. Zur Kenntnis Der nickelhydroxidelektrodeI.Über Das Nickel (II)-Hydroxidhydrat. Electrochim. Acta 1966, 11, 1079-1087.

(61) Li, Z. Q.; Lu, C. J.; Xia, Z. P.; Zhou, Y.; Luo, Z. X-Ray Diffraction Patterns of Graphite and Turbostratic Carbon. Carbon N. Y. 2007, 45, 1686-1695.

(62) McCrory, C. C. L.; Jung, S.; Peters, J. C.; Jaramillo, T. F. Benchmarking Heterogeneous Electrocatalysts for the Oxygen Evolution Reaction. J. Am. Chem. Soc. 2013, 135, 16977-16987.

(63) Frydendal, R.; Paoli, E. A.; Knudsen, B. P.; Wickman, B. B.; Malacrida, P.; Stephens, I. E. L.; Chorkendorff, I. Benchmarking the Stability of Oxygen Evolution Reaction Catalysts: The Importance of Monitoring Mass Losses. ChemElectroChem 2014, 1, 2075-2081.

(64) Shannon, R. D. Revised Effective Ionic Radii and Systematic Studies of Interatomic Distances in Halides and Chalcogenides. Acta Crystallogr. Sect. A 1976, 32, 751-767. 
(65) Klaus, S.; Cai, Y.; Louie, M. W.; Trotochaud, L.; Bell, A. T. Effects of Fe Electrolyte Impurities on $\mathrm{Ni}(\mathrm{OH})_{2} / \mathrm{NiOOH}$ Structure and Oxygen Evolution Activity. J. Phys. Chem. C 2015, 119, 7243-7254.

(66) Klaus, S.; Louie, M. W.; Trotochaud, L.; Bell, A. T. Role of Catalyst Preparation on the Electrocatalytic Activity of $\mathrm{Ni}_{(1-\mathrm{x})} \mathrm{Fe}_{\mathrm{x}} \mathrm{OOH}$ for the Oxygen Evolution Reaction. J. Phys. Chem. C 2015, 119, 18303-18316.

(67) Abidat, I.; Bouchenafa-Saib, N.; Habrioux, A.; Comminges, C.; Canaff, C.; Rousseau, J.; Napporn, T. W.; Dambournet, D.; Borkiewicz, O.; Kokoh, K. B. Electrochemically Induced Surface Modifications of Mesoporous Spinels $\left(\mathrm{Co}_{3} \mathrm{O}_{4-\delta}\right.$ , $\mathrm{MnCo}_{2} \mathrm{O}_{4-\delta}, \mathrm{NiCo}_{2} \mathrm{O}_{4-\delta}$ ) as the Origin of the OER Activity and Stability in Alkaline Medium. J. Mater. Chem. A 2015, 3, 17433-17444.

\section{Chapter IV}

(1) KREUTER, W.; Hofmann, H. Electrolysis: The Important Energy Transformer in a World of Sustainable Energy. Int. J. Hydrogen Energy 1998, 23, 661-666.

(2) Walter, M. G.; Warren, E. L.; McKone, J. R.; Boettcher, S. W.; Mi, Q.; Santori, E. A.; Lewis, N. S. Solar Water Splitting Cells. Chem. Rev. 2010, 110, 6446-6473.

(3) Galán-Mascarós, J. R. Water Oxidation at Electrodes Modified with EarthAbundant Transition-Metal Catalysts. ChemElectroChem 2015, 2, 37-50.

(4) Rüetschi, P.; Delahay, P. Influence of Electrode Material on Oxygen Overvoltage: A Theoretical Analysis. J. Chem. Phys. 1955, 23, 556.

(5) Trasatti, S. Electrocatalysis by Oxides - Attempt at a Unifying Approach. $J$. Electroanal. Chem. Interfacial Electrochem. 1980, 111, 125-131.

(6) Trotochaud, L.; Ranney, J. K.; Williams, K. N.; Boettcher, S. W. Solution-Cast Metal Oxide Thin Film Electrocatalysts for Oxygen Evolution. J. Am. Chem. Soc. 2012, 134, 17253-17261.

(7) Louie, M. W.; Bell, A. T. An Investigation of Thin-Film Ni-Fe Oxide Catalysts for the Electrochemical Evolution of Oxygen. J. Am. Chem. Soc. 2013, 135, 1232912337.

(8) Görlin, M.; Chernev, P.; Ferreira de Araújo, J.; Reier, T.; Dresp, S.; Paul, B.; Krähnert, R.; Dau, H.; Strasser, P. Oxygen Evolution Reaction Dynamics, Faradaic Charge Efficiency, and the Active Metal Redox States of Ni-Fe Oxide Water Splitting Electrocatalysts. J. Am. Chem. Soc. 2016, 138, 5603-5614. 
(9) Klaus, S.; Louie, M. W.; Trotochaud, L.; Bell, A. T. Role of Catalyst Preparation on the Electrocatalytic Activity of $\mathrm{Ni}_{(1-x)} \mathrm{Fe}_{\mathrm{x}} \mathrm{OOH}$ for the Oxygen Evolution Reaction. J. Phys. Chem. C 2015, 119, 18303-18316.

(10) Haber, J. A.; Cai, Y.; Jung, S.; Xiang, C.; Mitrovic, S.; Jin, J.; Bell, A. T.; Gregoire, J. M. Discovering Ce-Rich Oxygen Evolution Catalysts, from High Throughput Screening to Water Electrolysis. Energy Environ. Sci. 2014, 7, 682.

(11) Haber, J. A.; Anzenburg, E.; Yano, J.; Kisielowski, C.; Gregoire, J. M. Multiphase Nanostructure of a Quinary Metal Oxide Electrocatalyst Reveals a New Direction for OER Electrocatalyst Design. Adv. Energy Mater. 2015, 5 (10).

(12) Gregoire, J. M.; Xiang, C.; Mitrovic, S.; Liu, X.; Marcin, M.; Cornell, E. W.; Fan, J.; Jin, J. Combined Catalysis and Optical Screening for High Throughput Discovery of Solar Fuels Catalysts. J. Electrochem. Soc. 2013, 160, F337-F342.

(13) Burke, M. S.; Zou, S.; Enman, L. J.; Kellon, J. E.; Gabor, C. A.; Pledger, E.; Boettcher, S. W. Revised Oxygen Evolution Reaction Activity Trends for FirstRow Transition-Metal (Oxy)hydroxides in Alkaline Media. J. Phys. Chem. Lett. 2015, 6, 3737-3742.

(14) Wang, M.; Wang, Z.; Gong, X.; Guo, Z. The Intensification Technologies to Water Electrolysis for Hydrogen Production - A Review. Renew. Sustain. Energy Rev. 2014, 29, 573-588.

(15) Zeng, K.; Zhang, D. Recent Progress in Alkaline Water Electrolysis for Hydrogen Production and Applications. Prog. Energy Combust. Sci. 2010, 36, 307-326.

(16) Ayers, K. E.; Anderson, E. B.; Capuano, C.; Carter, B.; Dalton, L.; Hanlon, G.; Manco, J.; Niedzwiecki, M. Research Advances towards Low Cost, High Efficiency PEM Electrolysis. ECS Trans. 2010, 33, 3-15.

(17) Marini, S.; Salvi, P.; Nelli, P.; Pesenti, R.; Villa, M.; Berrettoni, M.; Zangari, G.; Kiros, Y. Advanced Alkaline Water Electrolysis. Electrochim. Acta 2012, 82, 384391.

(18) Shen, P. K.; Wang, C.-Y.; Jiang, S. P.; Sun, X.; Zhang, J. Electrochemical Energy: Advanced Materials and Technologies.

(19) Oliva, P.; Leonardi, J.; Laurent, J. F.; Delmas, C.; Braconnier, J. J.; Figlarz, M.; Fievet, F.; de Guibert, A. Review of the Structure and the Electrochemistry of Nickel Hydroxides and Oxy-Hydroxides. J. Power Sources 1982, 8, 229-255. 
(20) Pérez-Alonso, F. J.; Adán, C.; Rojas, S.; Peña, M. a.; Fierro, J. L. G. Ni/Fe Electrodes Prepared by Electrodeposition Method over Different Substrates for Oxygen Evolution Reaction in Alkaline Medium. Int. J. Hydrogen Energy 2014, $39,5204-5212$.

(21) Tao, Y.; Zaijun, L.; Ruiyi, L.; Qi, N.; Hui, K.; Yulian, N.; Junkang, L. Nickelcobalt Double Hydroxides Microspheres with Hollow Interior and Hedgehog-like Exterior Structures for Supercapacitors. J. Mater. Chem. 2012, 22, 23587.

(22) Hall, D. E. Ni ( OH ) 2 - Impregnated Anodes for Alkaline Water Electrolysis. J. Electrochem. Soc. 1983, 130, 317-321.

(23) Gong, M.; Li, Y.; Wang, H.; Liang, Y.; Wu, J. Z.; Zhou, J.; Wang, J.; Regier, T.; Wei, F.; Dai, H. An Advanced Ni-Fe Layered Double Hydroxide Electrocatalyst for Water Oxidation. J. Am. Chem. Soc. 2013, 135, 8452-8455.

(24) Ma, W.; Ma, R.; Wang, C.; Liang, J.; Liu, X.; Zhou, K.; Sasaki, T. A Superlattice of Alternately Stacked Ni-Fe Hydroxide Nanosheets and Graphene for Efficient Splitting of Water. ACS Nano 2015, 9, 1977-1984.

(25) Hall, D. E. Alkaline Water Electrolysis Anode Materials. J. Electrochem. Soc. 1985, 132, 41C-48C.

(26) Raney, M. Method of Producing Finely-Divided Nickel. Google Patents May 10, 1927.

(27) Wu, M.-S. S.; Huang, K.-C. C. Enhanced Electrochemical Performance of Nickel Hydroxide Electrode with Monolayer Hollow Spheres Composed of Nanoflakes. Int. J. Hydrogen Energy 2011, 36, 13407-13413.

(28) Wang, D.; Song, C.; Hu, Z.; Fu, X. Fabrication of Hollow Spheres and Thin Films of Nickel Hydroxide and Nickel Oxide with Hierarchical Structures. J. Phys. Chem. B 2005, 109, 1125-1129.

(29) Merrill, M.; Worsley, M.; Wittstock, A.; Biener, J.; Stadermann, M. Determination of the "NiOOH" Charge and Discharge Mechanisms at Ideal Activity. $J$. Electroanal. Chem. 2014, 717, 177-188.

(30) McCrory, C. C. L.; Jung, S.; Ferrer, I. M.; Chatman, S.; Peters, J. C.; Jaramillo, T. F. Benchmarking HER and OER Electrocatalysts for Solar Water Splitting Devices. J. Am. Chem. Soc. 2015, 137, 4347-4357.

(31) Batchellor, A. S.; Boettcher, S. W. Pulse-Electrodeposited Ni-Fe (Oxy)hydroxide Oxygen Evolution Electrocatalysts with High Geometric and Intrinsic Activities at Large Mass Loadings. ACS Catal. 2015, 5, 6680-6689. 
(32) Sabatier, P. Hydrogénations et Déshydrogénations Par Catalyse. Berichte der Dtsch. Chem. Gesellschaft 1911, 44, 1984-2001.

(33) Otagawa, T.; Bockris, J. O. Oxygen Evolution on Perovskites. J. Phys. Chem 1983, 87, 2960-2971.

(34) Grimaud, A.; May, K. J.; Carlton, C. E.; Lee, Y.-L.; Risch, M.; Hong, W. T.; Zhou, J.; Shao-Horn, Y. Double Perovskites as a Family of Highly Active Catalysts for Oxygen Evolution in Alkaline Solution. Nat. Commun. 2013, 4, 2439.

(35) Hong, W. T.; Risch, M.; Stoerzinger, K. a.; Grimaud, A.; Suntivich, J.; Shao-horn, Y. Toward the Rational Design of Non-Precious Transition Metal Oxides for Oxygen Electrocatalysis. Energy Environ. Sci. 2015, 13-15.

\section{Chapter V}

(1) Trotochaud, L.; Mills, T. J.; Boettcher, S. W. An Optocatalytic Model for Semiconductor-Catalyst Water-Splitting Photoelectrodes Based on In Situ Optical Measurements on Operational Catalysts. J. Phys. Chem. Lett. 2013, 4, 931-935.

(2) Walter, M. G.; Warren, E. L.; McKone, J. R.; Boettcher, S. W.; Mi, Q.; Santori, E. A.; Lewis, N. S. Solar Water Splitting Cells. Chem. Rev. 2010, 110, 6446-6473.

(3) McCrory, C. C. L.; Jung, S.; Peters, J. C.; Jaramillo, T. F. Benchmarking Heterogeneous Electrocatalysts for the Oxygen Evolution Reaction. J. Am. Chem. Soc. 2013, 135, 16977-16987.

(4) Sun, J.; Zhong, D. K.; Gamelin, D. R. Composite Photoanodes for Photoelectrochemical Solar Water Splitting. Energy Environ. Sci. 2010, 3, 1252.

(5) Klahr, B.; Gimenez, S.; Fabregat-Santiago, F.; Bisquert, J.; Hamann, T. W. Photoelectrochemical and Impedance Spectroscopic Investigation of Water Oxidation with "Co-Pi"-Coated Hematite Electrodes. J. Am. Chem. Soc. 2012, 134, 16693-16700.

(6) Seabold, J. A.; Choi, K.-S. Effect of a Cobalt-Based Oxygen Evolution Catalyst on the Stability and the Selectivity of Photo-Oxidation Reactions of a $\mathrm{WO}_{3}$ Photoanode. Chem. Mater. 2011, 23, 1105-1112.

(7) Liu, R.; Lin, Y.; Chou, L.-Y.; Sheehan, S. W.; He, W.; Zhang, F.; Hou, H. J. M.; Wang, D. Water Splitting by Tungsten Oxide Prepared by Atomic Layer Deposition and Decorated with an Oxygen-Evolving Catalyst. Angew. Chemie 2011, 123, 519-522. 
(8) Kay, A.; Cesar, I.; Grätzel, M. New Benchmark for Water Photooxidation by Nanostructured $\alpha-\mathrm{Fe}_{2} \mathrm{O}_{3}$ Films. J. Am. Chem. Soc. 2006, 128, 15714-15721.

(9) Tilley, S. D.; Cornuz, M.; Sivula, K.; Grätzel, M. Light-Induced Water Splitting with Hematite: Improved Nanostructure and Iridium Oxide Catalysis. Angew. Chemie 2010, 122, 6549-6552.

(10) Abdi, F. F.; van de Krol, R. Nature and Light Dependence of Bulk Recombination in Co-Pi-Catalyzed BiVO 4 Photoanodes. J. Phys. Chem. C 2012, 116, 9398-9404.

(11) Gamelin, D. R. Water Splitting: Catalyst or Spectator? Nat. Chem. 2012, 4, 965967.

(12) Zhong, D. K.; Gamelin, D. R. Photoelectrochemical Water Oxidation by Cobalt Catalyst ("Co-Pi") $/ \alpha-\mathrm{Fe}_{2} \mathrm{O}_{3}$ Composite Photoanodes: Oxygen Evolution and Resolution of a Kinetic Bottleneck. J. Am. Chem. Soc. 2010, 132, 4202-4207.

(13) McDonald, K. J.; Choi, K.-S. Photodeposition of Co-Based Oxygen Evolution Catalysts on $\alpha-\mathrm{Fe}_{2} \mathrm{O}_{3}$ Photoanodes. Chem. Mater. 2011, 23, 1686-1693.

(14) Barroso, M.; Cowan, A. J.; Pendlebury, S. R.; Grätzel, M.; Klug, D. R.; Durrant, J. R. The Role of Cobalt Phosphate in Enhancing the Photocatalytic Activity of $\alpha$ $\mathrm{Fe}_{2} \mathrm{O}_{3}$ toward Water Oxidation. J. Am. Chem. Soc. 2011, 133, 14868-14871.

(15) Steinmiller, E. M. P.; Choi, K.-S. Photochemical Deposition of Cobalt-Based Oxygen Evolving Catalyst on a Semiconductor Photoanode for Solar Oxygen Production. Proc. Natl. Acad. Sci. 2009, 106, 20633-20636.

(16) Surendranath, Y.; Bediako, D. K.; Nocera, D. G. Interplay of Oxygen-Evolution Kinetics and Photovoltaic Power Curves on the Construction of Artificial Leaves. Proc. Natl. Acad. Sci. U. S. A. 2012, 109, 15617-15621.

(17) Pijpers, J. J. H.; Winkler, M. T.; Surendranath, Y.; Buonassisi, T.; Nocera, D. G. Light-Induced Water Oxidation at Silicon Electrodes Functionalized with a Cobalt Oxygen-Evolving Catalyst. Proc. Natl. Acad. Sci. 2011, 108, 10056-10061.

(18) Reece, S. Y.; Hamel, J. A.; Sung, K.; Jarvi, T. D.; Esswein, A. J.; Pijpers, J. J. H.; Nocera, D. G.; Cook, T. R.; Barber, J.; Betley, T. A.; et al. Wireless Solar Water Splitting Using Silicon-Based Semiconductors and Earth-Abundant Catalysts. Science 2011, 334, 645-648.

(19) Young, E. R.; Costi, R.; Paydavosi, S.; Nocera, D. G.; Bulović, V.; Nozik, A. J.; Wrighton, M. S.; Heller, A.; Greatzel, M.; Lewis, N. S.; et al. Photo-Assisted Water Oxidation with Cobalt-Based Catalyst Formed from Thin-Film Cobalt Metal on Silicon Photoanodes. Energy Environ. Sci. 2011, 4, 2058. 
(20) Zhong, D. K.; Choi, S.; Gamelin, D. R. Near-Complete Suppression of Surface Recombination in Solar Photoelectrolysis by "Co-Pi" Catalyst-Modified W:BiVO 4 . J. Am. Chem. Soc. 2011, 133, 18370-18377.

(21) Ye, H.; Park, H. S.; Bard, A. J. Screening of Electrocatalysts for Photoelectrochemical Water Oxidation on W-Doped BiVO ${ }_{4}$ Photocatalysts by Scanning Electrochemical Microscopy. J. Phys. Chem. C 2011, 115, 1246412470.

(22) Gregoire, J. M.; Xiang, C.; Mitrovic, S.; Liu, X.; Marcin, M.; Cornell, E. W.; Fan, J.; Jin, J. Combined Catalysis and Optical Screening for High Throughput Discovery of Solar Fuels Catalysts. J. Electrochem. Soc. 2013, 160, F337-F342.

(23) Malara, F.; Cannavale, A.; Carallo, S.; Gigli, G. Smart Windows for Building Integration: A New Architecture for Photovoltachromic Devices. ACS Appl. Mater. Interfaces 2014, 6, 9290-9297.

(24) Dyer, A. L.; Bulloch, R. H.; Zhou, Y.; Kippelen, B.; Reynolds, J. R.; Zhang, F. A Vertically Integrated Solar-Powered Electrochromic Window for Energy Efficient Buildings. Adv. Mater. 2014, 26, 4895-4900.

(25) Seike, T.; Nagai, J. Electrochromism of 3d Transition Metal Oxides. Sol. Energy Mater. 1991, 22, 107-117.

(26) Hugot-Le Goff, A.; Cordoba de Toressi, S. No Title. In Proceedings of the Electrochemic Materials Symposium; The Electrochemical Society, 1990; p 157.

(27) Corrigan, D. A.; Knight, S. L. Electrochemical and Spectroscopic Evidence on the Participation of Quadrivalent Nickel in the Nickel Hydroxide Redox Reaction. $J$. Electrochem. Soc. 1989, 136, 613-619.

(28) McIntyre, J. D. E.; Kolb, D. M. Specular Reflection Spectroscopy of Electrode Surface Films. Symp. Faraday Soc. 1970, 4, 99.

(29) Decker, F.; Passerini, S.; Pileggi, R.; Scrosati, B. The Electrochromic Process in Non-Stoichiometric Nickel Oxide Thin Film Electrodes. Electrochim. Acta 1992, 37, 1033-1038.

(30) Trotochaud, L.; Ranney, J. K.; Williams, K. N.; Boettcher, S. W. Solution-Cast Metal Oxide Thin Film Electrocatalysts for Oxygen Evolution. J. Am. Chem. Soc. 2012, 134, 17253-17261.

(31) Trotochaud, L.; Young, S. L.; Ranney, J. K.; Boettcher, S. W. Nickel-Iron Oxyhydroxide Oxygen-Evolution Electrocatalysts: The Role of Intentional and Incidental Iron Incorporation. J. Am. Chem. Soc. 2014, 136, 6744-6753. 
(32) Azens, A.; Kullman, L.; Ragan, D. .; Granqvist, C. . Optically Passive Counter Electrodes for Electrochromic Devices: Transition Metal-cerium Oxide Thin Films. Sol. Energy Mater. Sol. Cells 1998, 54, 85-91.

(33) Monk, P. M. S.; Akhtar, S. P.; Boutevin, J.; Duffield, J. R. Toward the Tailoring of Electrochromic Bands of Metal-oxide Mixtures. Electrochim. Acta 2001, 46, 2091-2096.

(34) Smith, R. D. L.; Prévot, M. S.; Fagan, R. D.; Trudel, S.; Berlinguette, C. P. Water Oxidation Catalysis: Electrocatalytic Response to Metal Stoichiometry in Amorphous Metal Oxide Films Containing Iron, Cobalt, and Nickel. J. Am. Chem. Soc. 2013, 135, 11580-11586.

(35) Haber, J. A.; Cai, Y.; Jung, S.; Xiang, C.; Mitrovic, S.; Jin, J.; Bell, A. T.; Gregoire, J. M. Discovering Ce-Rich Oxygen Evolution Catalysts, from High Throughput Screening to Water Electrolysis. Energy Environ. Sci. 2014, 7, 682.

(36) Brückner, A. Simultaneous Combination of in Situ-EPR/UV-VIS/on Line GC: A Novel Setup for Investigating Transition Metal Oxide Catalysts under Working Conditions. Chem. Commun. 2001, 1, 2122.

(37) Brückner, A. Killing Three Birds with One Stone--Simultaneous Operando EPR/UV-vis/Raman Spectroscopy for Monitoring Catalytic Reactions. Chem. Commun. (Camb). 2005, No. 13, 1761-1763.

(38) Zeng, K.; Zhang, D. Recent Progress in Alkaline Water Electrolysis for Hydrogen Production and Applications. Prog. Energy Combust. Sci. 2010, 36, 307-326.

(39) Leenheer, A. J.; Atwater, H. a. Water-Splitting Photoelectrolysis Reaction Rate via Microscopic Imaging of Evolved Oxygen Bubbles. J. Electrochem. Soc. 2010, 157, B1290.

(40) Sides, P. J.; Tobias, C. W. Resistance of a Planar Array of Spheres : Gas Bubbles on an Electrode. 1981, 12, 2715-2720.

(41) Vogt, H.; Aras, Ö.; Balzer, R. J. The Limits of the Analogy between Boiling and Gas Evolution at Electrodes. Int. J. Heat Mass Transf. 2004, 47, 787-795.

(42) Balzer, R. J.; Vogt, H. Effect of Electrolyte Flow on the Bubble Coverage of Vertical Gas-Evolving Electrodes. J. Electrochem. Soc. 2003, 150, E11.

(43) Eigeldinger, J.; Vogt, H. The Bubble Coverage of Gas-Evolving Electrodes in a Flowing Electrolyte. Electrochim. Acta 2000, 45, 4449-4456. 